\title{
Determinantal Random Point Fields
}

\author{
Alexander Soshnikov \\ Caltech \\ Department of Mathematics \\ Sloan 253-37 \\ Pasadena, CA 91125, USA \\ sashas@gibbs1.caltech.edu \\ and \\ University of California, Davis* \\ One Shields Ave., \\ Davis, CA 95616, USA \\ soshniko@math.ucdavis.edu
}

\begin{abstract}
The paper contains an exposition of recent as well as sufficiently old results on determinantal random point fields . We start with some general theorems including the proofs of the necessary and sufficient condition for the existence of determinantal random point field with Hermitian kernel and a criterion for the weak convergence of its distribution. In the second section we proceed with the examples of the determinantal random fields from Quantum Mechanics, Statistical Mechanics, Random Matrix Theory, Probability Theory, Representation Theory and Ergodic Theory. In connection with the Theory of Renewal Processes we characterize all Hermitian determinantal random point fields in $\mathbb{R}^{1}$ and $\mathbb{Z}^{1}$ with independent identically distributed spacings. In the third section we study the translation invariant determinantal random point fields and prove the mixing property of any
\end{abstract}

*permanent address 
multiplicity and the absolute continuity of the spectra. In the last section we discuss the proofs of the the Central Limit Theorem for the number of particles in the growing box and the Functional Central Limit Theorem for the empirical distribution function of spacings.

\section{Definition and General Properties of De- terminantal Random Point Fields}

Let $E$ be a one-particle space and $X$ a space of finite or countable configurations of particles in $E$. In general $E$ can be a separable Hausdorff space, however for our purposes it is enough to consider

$$
E=\prod_{j=1}^{m} E_{j}, \text { where } E_{j} \cong \mathbb{R}^{d}\left(\text { or } \mathbb{Z}^{d}\right)
$$

If it is not mentioned specifically otherwise we always assume below $E=\mathbb{R}^{d}$ with the understanding that all resuls can be easily generalized to (1.1). We assume that each configuration $\xi=\left(x_{i}\right), x_{i} \in E, i \in \mathbb{Z}^{1}$ (or $\mathbb{Z}_{+}^{1}$ if $d>1$ ), is locally finite, that is for every compact $K \subset E \#_{K}(\xi)=\#\left(x_{i} \in K\right)$ is finite. The particles in $\xi$ are ordered in some natural way, e.g., $x_{i} \leq x_{i+1}$ for $d=1$, and if $d>1$ then either $x_{i}=x_{i+1}$, or

$$
\left|x_{i}\right|=\left(\sum_{j=1}^{d}\left(x_{i}^{(j)}\right)^{2}\right)^{\frac{1}{2}}<\left|x_{i+1}\right|=\left(\sum_{j=1}^{d}\left(x_{i+1}^{(j)}\right)^{2}\right)^{\frac{1}{2}}
$$

where $x_{i}=\left(x_{i}^{(1)}, \ldots, x_{i}^{(d)}\right)$, or $\left|x_{i}\right|=\left|x_{i+1}\right|$ and there exists $1 \leq r \leq d$ such that $x_{i}^{(j)} \leq x_{i+1}^{(j)}, 1 \leq j \leq r-1$, and $x_{i}^{(r)}<x_{i+1}^{(r)}$

To define a $\sigma$-algebra of measurable subsets of $X$ we first construct the so-called cylinder sets. Let $B \subset E$ be any bounded Borel set and $n \geq 0$. We call $C_{n}^{B}=\left\{\xi \in X: \#_{B}(\xi)=n\right\}$ a cylinder set. We define $\mathcal{B}$ as a $\sigma$-algebra generated by all cylinder sets (i.e., $\mathcal{B}$ is a minimal $\sigma$-algebra that contains all $\left.C_{n}^{B}\right)$.

Definition 1. A random point field is a triplet $(X, \mathcal{B}, P)$ where $P$ is a probability measure on $(X, \mathcal{B})$.

This definition raises a natural question, namely how one can construct such probability measures. The corresponding theory was developed by 
Lenard in [L1-L3] where a general case of $E$ locally compact Hausdorff space satisying the second axiom of countability was studied. If $E=\mathbb{R}^{d}$ or $\mathbb{Z}^{d}$ one can proceed quite naively by employing Kolmogorov's fundamental theorem from the theory of stochastic processes $([\mathrm{K}])$. Let $t$ and $s$ be two vectors from $E$ with rational coordinates $t=\left(t^{(1)}, \ldots, t^{(d)}\right), s=\left(s^{(1)}, \ldots, s^{(d)}\right)$. We denote an open rectangle $\left\{x=\left(x^{(1)}, \ldots x^{(d)}\right) \in E: x^{(j)}=t^{(j)}+\theta_{j}\left(s^{(j)}-t^{(j)}\right), 0<\right.$ $\left.\theta_{j}<1, j=1, \ldots, d\right\}$ by $\sqcap_{t, s}$. Let us denote the family of finite unions of open, closed or semi-closed rectangles with rational $t, s$ by $\mathcal{R}$. Suppose we are able to construct a joint distribution of non-negative integer-valued random variables $\eta_{D}, D \in \mathcal{R}$ (that we later identify with $\#_{D}$ ) such that the following finite-additivity condition holds

$$
\eta_{D}=\sum_{i=1}^{n} \eta_{D_{i}} \text { (a.e.) }
$$

if $D=\bigsqcup_{i=1}^{n} D_{i}, \quad D, D_{i} \in \mathcal{R}, i=1, \ldots n$. One immediately can replace (1.3) then by $\sigma$-additivity property

$$
\eta_{D}=\sum_{i=1}^{\infty} \eta_{D_{i}} \text { (a.e.) }
$$

$D=\bigsqcup_{i=1}^{\infty} D_{i}, D, D_{i} \in \mathcal{R}, i=1, \ldots$, (of course the fact that $\eta_{D}$ takes only non-negative integers is essential here!).

It is then easy to see that the joint distribution of random variables $\#_{D}=\eta_{D}, D \in \mathcal{R}$ with (1.3) (or (1.4) for that matter) uniquely defines a probability distribution on $(X, \mathcal{B})$.

Since in many cases it is convenient to define the distribution of random variables through their moments the following definition appears natural:

Definition 2. Locally integrable function $\rho_{k}: E^{k} \rightarrow \mathbb{R}_{+}^{1}$ is called the $k$-point correlation function of the random point field $(X, \mathcal{B}, P)$ if for any disjoint bounded Borel subset $A_{1}, \ldots, A_{m}$ of $E$ and $k_{i} \in \mathbb{Z}_{+}^{1}, i=1, \ldots m, \sum_{i=1}^{m} k_{i}=k$ the following identity holds:

$$
\mathbb{E} \prod_{i=1}^{m} \frac{\left(\#_{A_{i}}\right) !}{\left(\#_{A_{i}}-k_{i}\right) !}=\int_{A_{1}^{k_{1}} \times \cdots \times A_{m}^{k m}} \rho_{k}\left(x_{1}, \ldots, x_{k}\right) d x_{1} \ldots d x_{k}
$$


where by $\mathbb{E}$ we denote the mathematical expectation with respect to $P$. In particular $\rho_{1}(x)$ is the density of particles, since

$$
\mathbb{E} \#_{A}=\int_{A} \rho_{1}(x) d x
$$

for any bounded Borel $A \subset E$. In general $\rho_{k}\left(x_{1}, \ldots, x_{k}\right)$ has the following probabilistic interpretation: let $\left[x_{1}, x_{i}+d x_{i}\right], i=1, \ldots, k$ be infinitesimally small boxes around $x_{i}$, then $\rho_{k}\left(x_{1}, x_{2}, \ldots, x_{k}\right) d x_{1} \cdot \ldots \cdot d x_{k}$ is the probability to find a particle in each of these boxes. The problem of existence and uniqueness of a random point field defined by its correlation functions was studied in [L1-L3]. Not very surprisingly, Lenard's papers revealed many similarities to the classical moment problem ([A], [S2]). In particular the random point field is uniquely defined by its correlation functions if the distribution of random variables $\left\{\#_{A}\right\}$ is uniquely determined by its moments. The sufficient condition for the uniqueness derived in [L1] reads

$$
\sum_{k=0}^{\infty}\left(\frac{1}{(k+j) !} \int_{A^{k+j}} \rho_{k+j}\left(x_{1}, \ldots, x_{k+j}\right) d x_{1}, \ldots d x_{k+j}\right)^{-\frac{1}{k}}=\infty
$$

for any bounded Borel $A \subset E$ and any integer $j \geq 0$, however we invite the reader to check that the divergence of the series with $j=0$, namely

$$
\sum_{k=0}^{\infty}\left(\frac{1}{k !} \int_{A^{k}} \rho_{k}\left(x_{1}, \ldots, x_{k}\right) d x_{1}, \ldots d x_{k}\right)^{-\frac{1}{k}}=\infty
$$

implies (1.6) for any $j \geq 0$. In [L2], [L3] Lenard obtained the necessary and sufficient condition for the existence of a random point field with the prescribed correlation functions.

Theorem 1. (Lenard)

Locally integrable functions $\rho_{k}: E^{k} \rightarrow \mathbb{R}^{1}, k=1,2, \ldots$ are the correlation functions of some random point field if and only if the Symmetry and Positivity Conditions below are satisfied.

\section{a) Symmetry Condition}

$\rho_{k}$ is invariant under the action of the symmetric group $S_{k}$, i.e.,

$$
\rho_{k}\left(x_{\sigma(1)}, \ldots, x_{\sigma(k)}\right)=\rho_{k}\left(x_{1}, \ldots, x_{k}\right)
$$

for any $\sigma \in S_{k}$. 


\section{b) Positivity Condition}

For any finite set of measurable bounded functions $\varphi_{k}: E^{k} \rightarrow \mathbb{R}^{1}$, $k=0,1, \ldots, N$ with compact support, such that

$$
\varphi_{0}+\sum_{k=1}^{N} \sum_{i_{1} \neq \cdots \neq i_{k}} \varphi_{k}\left(x_{i_{1}}, \ldots, x_{i_{k}}\right) \geq 0
$$

for all $\xi=\left(x_{i}\right) \in X$, the next inequality must be valid:

$$
\varphi_{0}+\sum_{k=1}^{N} \int_{E^{k}} \varphi_{k}\left(x_{1}, \ldots, x_{k}\right) \rho_{k}\left(x_{1}, \ldots, x_{k}\right) d x_{1} \ldots d x_{k} \geq 0 .
$$

The necessary part of the theorem is quite easy since both conditions have an obvious probabilistic interpretation. In particular the Positivity Condition means that the mathematical expectations of a certain class of non-negative random variables must be non-negative. The sufficient part is more elaborate and relies on an analogue of the Riesz Representation Theorem and the RieszKrein Extension Theorem (a close relative of the Hahn-Banach Theorem). It should be noted that Lenard established his results in a general setting when $E$ is locally compact Hausdorff space with the second axiom of countability.

One can obtain a slightly weaker (but still hopelessly ineffective!) variant of the Positivity Condition by approximating $\varphi_{k}$ from above by step functions. Let $\mathcal{P}_{k}$ be the class of polynomials in $k$ variables that take non-negative values on non-negative integers. Since the polynomials $\left\{\prod_{i=1}^{k} \prod_{j=0}^{m_{i}-1}\left(x_{i}-j\right), m_{i} \geq\right.$ $0\}$ form a linear basis in the vector space of all polynomials in $k$ variables, we can represent any $q\left(x_{1}, \ldots, x_{k}\right) \in \mathcal{P}_{k}$ as

$$
q\left(x_{1}, \ldots x_{k}\right)=\sum_{m_{1}, \ldots, m_{k} \geq 0} a_{m_{1}, \ldots, m_{k}} \cdot \prod_{i=1}^{k} \prod_{j=0}^{m_{i}-1}\left(x_{i}-j\right)
$$

Positivity Condition*: For any $q \in \mathcal{P}_{k}, k \geq 1$, any bounded Borel sets $A_{1}, \ldots, A_{k} \subset E$, the following condition must be satisfied:

$$
a_{0, \ldots, 0}+\sum_{m \geq 1} \sum_{m_{1}+\cdots+m_{k}=m} a_{m_{1}, \ldots, m_{k}} \int_{\prod_{i=1}^{k} A_{i}^{m_{i}}} \rho_{m}\left(x_{1}, \ldots, x_{m}\right) d x_{1} \ldots d x_{m} \geq 0 .
$$


Indeed, the 1.h.s. at (1.11) is equal to

$$
\begin{gathered}
\mathbb{E} q\left(\#_{A_{1}}, \ldots, \#_{A_{k}}\right)=\mathbb{E}\left[a_{0, \ldots 0}+\sum_{m \geq 1} \sum_{m_{1}+\cdots+m_{k}=m} a_{m_{1}, \ldots, m_{k}} \cdot \sum_{i_{1} \neq \cdots \neq i_{m}}\right. \\
\left.\chi_{A_{1}^{m_{1}} \times \cdots \times A_{k}^{m_{k}}}\left(x_{i_{1}}, \ldots, x_{i_{m}}\right)\right]
\end{gathered}
$$

One can notice that in a sense the Positivity Condition* is similar to the condition on the moments of the integer-valued nonnegative random variable.

In our paper we will study a special class of random point fields introduced by Macchi in [Ma] (see also [DVJ]). We start with an integral operator $K$ : $L^{2}\left(\mathbb{R}^{d}\right) \rightarrow L^{2}\left(\mathbb{R}^{d}\right)$ that we assume to be non-negative and locally trace class. The last condition means that for any compact $B \subset \mathbb{R}^{d}$ the operator $K \cdot \chi_{B}$ is trace class, where $\chi_{B}(x)$ is an indicator of $B$. Therefore we have

$$
K \geq 0, \quad \operatorname{Tr}\left(\chi_{B} K \cdot \chi_{B}\right)<+\infty
$$

The kernel of $K$ is defined up to a set of measure zero in $\mathbb{R}^{d} \times \mathbb{R}^{d}$. For our purposes it is convenient to choose it in such a way that for any bounded measurable $B$ and any positive integer $n$

$$
\operatorname{Tr}\left(\left(\chi_{B} K \chi_{B}\right)\right)=\int_{B} K(x, x) d x
$$

It appears that one can indeed achieve this. We start with

Lemma 1. ([S3], [AvSS] Remark 3.4) Let $K$ be trace class on $L^{2}\left(\mathbb{R}^{d}\right)$. Then its integral kernel may be chosen so that the function $M(x, y) \equiv K(x, x+y)$ is a continuous function of $y$ with values in $L^{1}\left(\mathbb{R}^{d}\right)$. Furthermore if $m(y)=$ $\int M(x, y) d x$, then $\operatorname{Tr} K=m(0)=\int K(x, x) d x$.

Proof. We give the proof only when $K$ is non-negative. The general case is quite similar. Let $\left\{\lambda_{j}\right\}_{j \geq 1}$ is the set of non-zero eigenvalues of $K$ and $\left\{\varphi_{j}\right\}_{j \geq 1}$ is the set of the corresponding eigenfunctions. The canonical form of $K$ (as a selfadjoint compact operator) is

$$
K=\sum_{j \geq 1} \lambda_{j} \cdot\left(\varphi_{j}, \cdot\right) \cdot \varphi_{j}
$$

Fix $y \in \mathbb{R}^{d}$ and consider $M(x, y)=\sum_{j=1}^{\infty} \lambda_{j} \cdot \varphi_{j}(x) \cdot \overline{\varphi_{j}(x+y)}$ as a function of $x$. Since $\left\|\varphi_{j}(\cdot) \cdot \overline{\varphi_{j}(\cdot+y)}\right\|_{1}=\int_{\mathbb{R}^{d}}\left|\varphi_{j}(x) \cdot \overline{\varphi_{j}(x+y)}\right| d x \leq\left\|\varphi_{j}\right\|_{2} \cdot\left\|\varphi_{j}\right\|_{2}=1$, 
the series defining $M(\cdot, y)$ converges in $L^{1}\left(\mathbb{R}^{d}\right)$ for any $y$ and $\|M(\cdot, y)\|_{1} \leq$ $\sum_{j=1}^{\infty} \lambda_{j}=\operatorname{Tr} K<+\infty$. If we now consider $K(x, y) \equiv M(x, y-x)$, it is well defined for a.e. $(x, y) \in \mathbb{R}^{d} \times \mathbb{R}^{d}$ and gives a kernel for $K$. The $L^{1}$-continuity of $M(\cdot, y)$ follows from $\left\|\sum_{j \geq 1} \lambda_{j}\left(\varphi_{j}(\cdot) \overline{\varphi_{j}\left(\cdot+y_{1}\right)}-\varphi_{j}(\cdot) \cdot \overline{\varphi_{j}\left(\cdot+y_{2}\right)}\right)\right\|_{1} \leq \sum_{j=1}^{N} \lambda_{j}$. $\left\|\varphi_{j}\right\|_{2} \cdot\left\|\varphi_{j}\left(\cdot+y_{1}\right)-\varphi_{j}\left(\cdot+y_{2}\right)\right\|_{2}+\sum_{j \geq N} \lambda_{j}$. Choosing $N$ sufficiently large one can make $\sum_{j \geq N} \lambda_{j}<\frac{\epsilon}{2}$. Choosing $y_{1}$ sufficiently close to $y_{2}$ so that for each $1 \leq j \leq N\left\|\varphi_{j}(\cdot)-\varphi_{j}\left(\cdot+y_{2}-y_{1}\right)\right\|_{2} \leq \frac{\epsilon}{2 \cdot \sum_{j=1}^{N} \lambda_{j}}$ we have the first term less than $\frac{\epsilon}{2}$ as well.

With the help of Lemma 1 we derive

Lemma 2. Let $K$ be a non-negative locally trace class operator on $L^{2}\left(\mathbb{R}^{d}\right)$. Then its integral kernel can be chosen in such a way that for any bounded measurable $B \subset \mathbb{R}^{d}$ the function

$$
M_{B}(x, y)=\left(K \cdot \chi_{B}\right)(x, x+y)
$$

is a continuous function of $y$ with values in $L^{1}(B)$. Furthermore,

$$
\operatorname{Tr}\left(\chi_{B} K \chi_{B}\right)=\int_{B} K(x, x) d x
$$

Proof. Let $K_{n}=\chi_{[-n, n]^{d}} \cdot K \cdot \chi_{[-n, n]^{d}}$. By Lemma 1 one can choose the kernel $K_{n}(x, y)$, such that $K_{n}(\cdot, \cdot+y)$ is continuous in $L^{1}\left([-n, n]^{d}\right)$-norm. We denote $M_{n}(x, y)=K_{n}(x, x+y)$. Since $K_{n+1}(x, y)=K_{n}(x, y)$ for almost all $(x, y) \in$ $[-n, n]^{d} \times[-n, n]^{d}$ we conclude that for almost all $|y| \leq n M_{n+1}(x, y)=$ $M_{n}(x, y)$ for a.e. $|x| \leq n-|y|$. The $L^{1}$-continuity of $M_{n+1}(\cdot, y), M_{n}(\cdot, y)$ allows to replace "almost all $|y| \leq n$ " by "all $|y| \leq n$ ". Therefore for any $y$ the values of $M_{n}(x, y)$ eventually agree for a.e. $x$. We denote this value by $M(x, y)$. The function $M(\cdot, y)$ inherits local $L^{1}$-continuity from $\left\{M_{n}(\cdot, y)\right\}$ and (1.14) follows. It may be worthwhile to note that for any positive integer $k$ and bounded measurable $B_{1} \subset \mathbb{R}^{d}, \ldots, B_{k} \subset \mathbb{R}^{d}$ the kernel

$$
M_{B}^{(k)}(x, y)=\left(\left(K \cdot \chi_{B_{1}}\right) \cdot \ldots \cdot\left(K \cdot \chi_{B_{k}}\right)\right)(x, x+y)
$$


is also a continuous function of y with the values in $L^{1}\left(\mathbb{R}^{d}\right)$ and $\operatorname{Tr}\left(K \cdot \chi_{B_{1}}\right) \cdot \ldots \cdot\left(K \cdot \chi_{B_{k}}\right)=\int_{B_{1} \times \cdots \times B_{k}} K\left(x_{1}, x_{2}\right) \cdot \ldots \cdot K\left(x_{k}, x_{1}\right) d x_{1} \ldots d x_{k}$,

in particular

$$
\operatorname{Tr}\left(K \cdot \chi_{B}\right) \cdot \ldots \cdot\left(K \cdot \chi_{B}\right)=\int_{B^{k}} K\left(x_{1}, x_{2}\right) \cdot \ldots \cdot K\left(x_{k}, x_{1}\right) d x_{1} \ldots d x_{k} .
$$

Indeed, for any two versions of the integral kernel and $k>1$ the expressions in the last two integrals coincide up to a set of measure zero. Since we have already proved that there exists the kernel of

$$
K \cdot \chi_{B_{1}} \ldots K \cdot \chi_{B_{k}}
$$

satisfying the $L^{1}$ - continuity condition from Lemma 2 , the same condition is satisfied by any variant of the kernel.

Definition 3. A random point field in $E$ is called determinantal (or fermion) if its $n$-point correlation functions are given by

$$
\rho_{n}\left(x_{1}, \ldots, x_{n}\right)=\operatorname{det}\left(K\left(x_{i}, x_{j}\right)\right)_{1 \leq i \leq n}
$$

In the case $E=\bigsqcup_{j=1}^{M} E_{j}, E_{j} \cong \mathbb{R}^{d}$ the definition takes the following form: Let $K$ be a trace class operator on $L^{2}\left(\mathbb{R}^{d}\right) \oplus \cdots \oplus L^{2}\left(\mathbb{R}^{d}\right)$. Then $K$ has a matrix valued kernel $\left(K_{r s}(x, y)\right)_{1 \leq r, s \leq m}, x, y \in \mathbb{R}^{d}$.

Definition 3'. A random point field in $E$ is called determinantal (or fermion) if its $n$-point correlation functions are given by

$\rho_{n}\left(x_{11}, x_{12}, \ldots x_{1 i_{1}}, \ldots, x_{m 1}, x_{m 2}, \ldots, x_{m i_{m}}\right)=\operatorname{det}\left(K_{r s}\left(x_{r i}, x_{s j}\right)\right)_{\substack{1 \leq i \leq i_{r}, r=1, \ldots, m \\ 1 \leq j \leq i_{s}, s=1, \ldots, m}}$,

where $n=i_{1}+i_{2}+\cdots+i_{m}, x_{r i} \in E_{r}, 1 \leq r \leq m, 1 \leq i \leq i_{r}$.

Remark 1. If the kernel is Hermitian-symmetric then the non-negativity of $n$-point correlation functions implies that the kernel $K(x, y)$ is non-negative 
definite and therefore indeed $K$ must be a non-negative operator. It should be noted however that there exist determinantal random point fields corresponding to non-Hermitian kernels (see the remark after (1.36) and the examples in the sections 2.2 and 2.5).

Remark 2. The condition (1.13) is satisfied for all continuous non-negative definite kernels (see [GK], section III.10 or [RS], vol. III, section XI.4). In general situation when $K(x, x)$ is locally integrable, non-negative definiteness of $K(x, y)$ implies that $K_{B}$ is a Hilbert-Schmidt operator and one can use a theorem of Gohberg-Krein ([GK], section III.10, theorem 10.1) that claims that a non-negative Hilbert-Schmidt operator A is trace class iff

$$
\varlimsup_{h \rightarrow 0} \frac{1}{(2 h)^{2 d}} \int \prod_{j=1}^{d}\left[2 h-\left|x^{j}-y^{j}\right|\right]_{+} A(x, y) d x d y<\infty
$$

where $t_{+}=\max (t, 0), x=\left(x^{1}, \ldots, x^{d}\right), y=\left(y^{1}, \ldots y^{d}\right)$, and TrA is then given by (1.22).

An interesting generalization of determinantal random point fields, so called immanantal random point fields (processes) was introduced by Diaconis and Evans in [DE].

The classical formula of Fredholm (see [S1], Chapter 3) claims that a trace class operator with a continuous (in a usual sense) kernel satisfies

$$
\operatorname{Tr}\left(\wedge^{n}(A)\right)=\frac{1}{n !} \int \operatorname{det}\left(A\left(x_{i}, x_{j}\right)\right)_{1 \leq i, j \leq n} d x_{1}, \ldots d x_{n}
$$

In general the kernel $K(x, y)$ may not be continuous, however (1.18) and the Lidskii theorem (see e.g., [RS], volume IV, section XIII.17 or [S1], Theorem 3.7) imply

$$
\begin{gathered}
\int_{B^{n}} K\left(x_{1}, x_{2}\right) \cdot \ldots \cdot K\left(x_{n}, x_{1}\right) d x_{1} \ldots d x_{n}=\sum_{j=1}^{\infty} \lambda_{j}^{n}\left(K_{B}\right), \\
\operatorname{Tr}\left(\wedge^{n}\left(K_{B}\right)\right)=\sum_{j_{1}<\cdots<j_{n}} \lambda_{j_{1}}\left(K_{B}\right) \cdot \ldots \cdot \lambda_{j_{n}}\left(K_{B}\right)
\end{gathered}
$$

Combining (1.24) and (1.25) one arrives at

$$
\operatorname{Tr}\left(\wedge^{n}\left(K_{B}\right)\right)=\frac{1}{n !} \int_{B^{n}} \operatorname{det}\left(K\left(x_{i}, x_{j}\right)\right)_{1 \leq i, j \leq n} d x_{1}, \ldots d x_{n}
$$


It follows then from (1.17) that

$$
\begin{aligned}
& \operatorname{Tr}\left(\left(K \cdot \chi_{B_{1}}\right) \wedge \cdots \wedge\left(K \cdot \chi_{B_{n}}\right)\right)= \\
& \quad \frac{1}{n !} \int \operatorname{det}\left(K\left(x_{i}, x_{j}\right) \cdot \chi_{B_{j}}\left(x_{j}\right)\right)_{1 \leq i, j \leq n} d x_{1} \ldots d x_{n}
\end{aligned}
$$

Definition 4. Let the kernel $K$ as in Lemma 2. We say that it defines a determinantal random point field $(X, B, P)$ if $(1.21)$ holds.

Theorem 2. Let $(X, B, P)$ be a determinantal random point field with the kernel $K$. For any finite number of disjoint bounded Borel sets $B_{j} \subset$ $E, j=1, \ldots, n$, the generating function of the probability distribution of $\#_{B_{j}}=\#\left\{x_{i} \in B_{j}\right\}$ is given by

$$
\mathbb{E} \prod_{j=1}^{n} z_{j}^{\#_{B_{j}}}=\operatorname{det}\left(\mathrm{Id}+\chi_{B} \sum_{j=1}^{n}\left(z_{j}-1\right) \cdot K \cdot \chi_{B_{j}}\right)
$$

Remark 3. (1.28) is the equality of two entire functions. The r.h.s. of (1.28) is well defined as a Fredholm determinant of a trace class operator (see e.g., [RS], volume IV, section XIII.17 or [S1], section 3).

Recall that by definition

$$
\mathbb{E} \prod_{j=1}^{n} z^{\# B_{j}}=\sum_{k_{1}, \ldots, k_{n}=0}^{\infty} P\left(\#_{B_{j}}=k_{j}, j=1, \ldots, n\right) \cdot \prod_{j=1}^{n} z_{j}^{k_{j}}
$$

and

$$
\begin{gathered}
\operatorname{det}\left(\operatorname{Id}+\chi_{B} \sum_{j=1}^{n}\left(z_{j}-1\right) \cdot K \cdot \chi_{B_{j}}\right)=1+\sum_{m=1}^{\infty} \sum_{j_{1}, \ldots j_{m}=1}^{n} \prod_{\ell=1}^{m} \\
\left(z_{j_{\ell}}-1\right) \cdot \operatorname{Tr}\left(\chi_{B} \cdot K \cdot \chi_{B_{j_{1}}} \wedge \cdots \wedge \chi_{B} K \cdot \chi_{B_{j_{m}}}\right)
\end{gathered}
$$

Proof of Theorem 2. The Taylor expansion of the generating function near $\left(z_{1}, \ldots, z_{n}\right)=(1, \ldots, 1)$ is given by

$$
\mathbb{E} \prod_{j=1}^{n} z_{j}^{\#_{B_{j}}}=1+\sum_{m=1}^{\infty} \sum_{m_{1}+\cdots+m_{n}=m} \mathbb{E} \prod_{j=1}^{n} \frac{\left(\#_{B_{j}}\right) !}{\left(\#_{B_{j}}-m_{j}\right) ! \cdot\left(m_{j}\right) !} \cdot \prod_{j=1}^{n}\left(z_{j}-1\right)^{m_{j}}
$$


The radius of convergence of (1.30) is infinite since

$$
\operatorname{Tr}\left(K \cdot \chi_{B_{j_{1}}} \wedge \cdots \wedge K \cdot \chi_{B_{j_{m}}} \leq \frac{1}{m !} \operatorname{Tr}\left(K \cdot \chi_{B}\right)^{m}, \text { where } B=\bigsqcup_{j=1}^{n} B_{j}\right.
$$

Therefore, it is enough to show that the coefficients in the series (1.30), (1.31) coincide. The case $n=1$ follows then from (1.5), (1.21), (1.26). Using (1.27) instead of (1.26) we prove the case $n \geq 1$ as well.

Remark 4. Theorem 2 is well known in the Theory of Random Point Fields (see [DVJ], p. 140, exercise 5.4.9) and in the Random Matrix Theory (see [TW1]).

As we already mentioned above, if an operator $K$ defines a determinantal random point field it must be non-negative because of the non-negativity of the correlation functions. It follows from Theorem 2, formula (1.28) that $K$ must also be bounded from above by the identity operator, i.e., $K \leq 1$. Indeed, suppose $\|K\|>1$. Then there exists a bounded Borel $B \subset E$ such that $\left\|K_{B}\right\|>1+\frac{\|K\|-1}{2}>1$. Let $\lambda_{1}\left(K_{B}\right) \geq \lambda_{2}\left(K_{B}\right) \geq \lambda_{3}\left(K_{B}\right) \geq \ldots$ be the eigenvalues of $K_{B}$ and choose $0<z_{0}<1$ so that $1+\left(z_{0}-1\right) \cdot \lambda_{1}\left(K_{B}\right)=0$. Then $\mathbb{E} z_{0}^{\#_{B}}=\sum_{k=1}^{\infty} P\left(\#_{B}=k\right) z_{0}^{k}=\operatorname{det}\left(\mathrm{Id}+\left(z_{0}-1\right) \cdot K_{B}\right)=($ by Theorem XIII.106 from $[\mathrm{RS}])=\prod_{j>1}\left(1+\left(z_{0}-1\right) \cdot \lambda_{j}\left(K_{B}\right)\right)=0$. Therefore $P\left(\#_{B}=k\right)=0$ for any $k$, a contradiction. On the other side assume $0 \leq K \leq 1$ and let (1.28) define what we hope to be the distribution of non-negative integer-valued random variables $\left\{\#_{B}\right\}$.

Lemma 3. Let $0 \leq K \leq 1$ and $K$ be a locally trace class operator. Then (1.28) defines the distribution of non-negative integer-valued random variables $\left\{\#_{B}\right\}$ with the additional property that for $B=\bigsqcup_{i=1}^{n} B_{i}$

$$
\#_{B}=\sum_{i=1}^{n} \#_{B_{i}} \text { (a.e.). }
$$

We need to show three things: first, that (1.28) defines some finitedimensional distributions; second, that the finite-dimensional distributions satisfy the additivity property (1.33); and third, that the finite-dimensional distributions are consistent and therefore we can apply the Kolmogorov's Fundamental Theorem to prove the existence of the distribution of $\left\{\#_{B}\right\}$. Since the Fredholm determinant in (1.28) is 1 when $z_{i}=1, i=1, \ldots, n$, 
the first statement would follow from the non-negativity of the Taylor coefficients of the Fredholm determinant at $z_{i}=0, i=1, \ldots, n$. Consider $0 \leq z_{i} \leq 1, i=1, \ldots, n$ and assume for a moment $\|K\|<1$ (the case $\|K\|=1$ would be treated later by a limiting argument). Let $B=\bigsqcup_{i=1}^{n} B_{i}$. Then $\left\|K_{B}\right\|<1$ and $\left(\mathrm{Id}-K_{B}\right)^{-1}$ is a bounded linear operator such that $\left(\mathrm{Id}-K_{B}\right)^{-1}-\mathrm{Id}=K_{B} \cdot\left(\mathrm{Id}-K_{B}\right)^{-1}$ is trace class. Applying Theorem XIII.p105 from $[\mathrm{RS}]$, vol. IV we obtain

$$
\begin{aligned}
& \operatorname{det}\left(\operatorname{Id}+\chi_{B} \sum_{j=1}^{n}\left(z_{j}-1\right) \cdot K \cdot \chi_{B_{j}}\right)=\operatorname{det}\left(( \operatorname { I d } - K _ { B } ) \cdot \left(\operatorname{Id}+\sum_{j=1}^{n} z_{j} \cdot\right.\right. \\
& \left.\left.\left(\operatorname{Id}-K_{B}\right)^{-1} \cdot \chi_{B} \cdot K \cdot \chi_{B_{j}}\right)\right)=\operatorname{det}\left(\operatorname{Id}-K_{B}\right) \cdot \operatorname{det}\left(\operatorname{Id}+\sum_{j=1}^{n} z_{j} \cdot\right. \\
& \left.\left(\operatorname{Id}-K_{B}\right)^{-1} \cdot \chi_{B} K \cdot \chi_{B_{j}}\right)=\operatorname{det}\left(\operatorname{Id}-K_{B}\right) \cdot \sum_{k=1}^{\infty} \operatorname{Tr}\left(\wedge ^ { k } \left(\sum_{j=1}^{n} z_{j} \cdot\right.\right. \\
& \left.\left.\left(\operatorname{Id}-K_{B}\right)^{-1} \cdot \chi_{B} K \cdot \chi_{B_{j}}\right)\right)=\sum_{k_{1}, \ldots, k_{n} \geq 0} \frac{\left(k_{1}+\cdots+k_{n}\right) !}{k_{1} ! \ldots k_{n} !} \prod_{j=1}^{n} \\
& z_{j}^{k_{j}} \cdot \operatorname{det}\left(\operatorname{Id}-K_{B}\right) \cdot \operatorname{Tr}\left(\wedge_{j=1}^{n}\left(\wedge^{k_{j}}\left(\chi_{B_{j}}\left(\operatorname{Id}-K_{B}\right)^{-1} \cdot \chi_{B} K \cdot \chi_{B_{j}}\right)\right)\right)
\end{aligned}
$$

One can see from (1.34) that Taylor coefficients are, up to some positive factors, the traces of the exterior products of the non-negative operators, and, therefore, non-negative. We conclude that (1.28) defines some finitedimensional distributions. Since $\mathbb{E} \prod_{i=1}^{n} z^{\#_{B_{i}}}=\operatorname{det}\left(\operatorname{Id}+\chi_{B} \sum_{i=1}^{n}(z-1) K \chi_{B_{i}}\right)=$ $\operatorname{det}\left(\operatorname{Id}+(z-1) \cdot K_{B}\right)=\mathbb{E} z^{\#_{B}}$, we conclude that $\#_{B}=\sum_{i=1}^{n} \#_{B_{i}}$ (a.e.). The formula (1.28) defined the finite dimensional distributions of $\#_{B_{i}}$ for disjoint compact sets. In the case of non-empty self-intersections one represents $B_{i}$ as $\sqcup C_{k_{i}}$, where $\left\{C_{k}\right\}$ are disjoint sets, defines distributions of $\#_{C_{k}}$ and then uses the additivity property (1.33) to define the distributions of $\#_{B_{i}}$. To prove the consistency of the finite-dimensional distributions we note that (1.33) allows us to check it only for the disjoint $B_{1}, \ldots, B_{n+1}$. But then it trivially follows from $\operatorname{det}\left(\mathrm{Id}+\chi_{B} \sum_{j=1}^{n}\left(z_{j}-1\right) \cdot K \cdot \chi_{B_{j}}+\chi_{B}(1-1) \cdot K \cdot \chi_{B_{n+1}}\right)=$ $\operatorname{det}\left(\operatorname{Id}+\chi_{B} \sum_{j=1}^{n}\left(z_{j}-1\right) \cdot K \cdot \chi_{B_{j}}\right)$. The case $\|K\|<1$ is proven. Now let $\|K\|=1$. Denote by $K^{(\epsilon)}:=K \cdot(1-\epsilon), \epsilon>0$ and $\#_{B}^{(\epsilon)}$ the random variables corresponding to the kernel $K^{(\epsilon)}$. Since $\left\|K^{(\epsilon)}\right\|<1$ the arguments above establish the result of Lemma 3 for $K^{(\epsilon)}$. It is an easy exercise to see that 
$\mathbb{E} \prod_{i=1}^{n} z_{i}^{\# B_{B_{i}}^{(\epsilon)}}=1+\sum_{m=1}^{\infty} \sum_{m_{1}+\cdots+m_{n}=m} \mathbb{E} \prod_{j=1}^{n} \frac{\left(\#_{B_{j}^{(\epsilon)}}\right) !}{\left(\#_{B_{j}^{(\epsilon)}}-m_{j}\right) ! \cdot\left(m_{j}\right) !} \cdot \prod_{j=1}^{n}\left(z_{1}-1\right)^{m_{j}}$ uniformly converges with all derivatives to $\mathbb{E} \prod_{i=1}^{n} z_{i}^{\#_{B_{i}}}$ on compact sets as $\epsilon \rightarrow 0$.

Lemma 3 is proven.

The results above prove

Theorem 3. Hermitian locally trace class operator $K$ on $L^{2}(E)$ defines a determinantal random point field if and only if $0 \leq K \leq 1$. If the corresponding random point field exists it is unique.

The necessary and sufficient condition for the existence of the field has been already established. The uniqueness result easily follows from the general criterion (1.6') since $\frac{1}{k !} \int_{A^{k}} \rho_{k}\left(x_{1}, \ldots, x_{k}\right) d x_{1} \ldots d x_{k}=\operatorname{Tr}\left(\wedge^{k}\left(K_{A}\right)\right) \leq \frac{\operatorname{Tr}\left(K_{A}\right)^{k}}{k !} \leq$ $\frac{1}{k !}$

Consider arbitrary bounded Borel set $B \subset E$. Then $\operatorname{Tr}\left(K_{B}\right)=\mathbb{E} \#_{B}<\infty$ and the number of particles in $B$ is finite with probability 1 . Let us write $X=\bigsqcup_{0 \leq k<\infty} C_{k}^{B}$, where as before $C_{k}^{B}=\left\{\xi \in X: \#_{B}(\xi)=k\right\}$. We choose a kernel for $\chi_{B} \cdot K \cdot \chi_{B}$ in such a way (see Lemma 1) that $\left(\chi_{B} \cdot K \cdot \chi_{B}\right)(x, x+y)=$ $\sum_{i=1}^{\infty} \lambda_{i}(B) \varphi_{i}(x) \cdot \overline{\varphi_{i}(x+y)}$ is a continuous function of $y$ in $L^{1}(B)$ norm. Assume for a moment that $K_{B}<1$. Then

$$
L_{B}(x, x+y)=\sum_{i=1}^{\infty} \frac{\lambda_{i}(B)}{1-\lambda_{i}(B)} \cdot \varphi_{i}(x) \cdot \overline{\varphi_{i}(x+y)}
$$

is also a continuous function of $y$ in $L^{1}(B)$ norm and is a kernel of $L_{B}=$ $\left(\mathrm{Id}-K_{B}\right)^{-1} K_{B}$. Taking $B_{j}$ in (1.34) infinitesimally small one concludes that for each $C_{k}^{B}$ the distribution of $k$ particles $x_{1} \leq x_{2} \leq \cdots \leq x_{k}$ in $B$ has a density with respect to the Lebesgue measure. Denoting this density by $p_{k}\left(x_{1}, \ldots, x_{k}\right)$ we obtain

$$
p_{k}\left(x_{1}, \ldots, x_{k}\right)=\operatorname{det}\left(\operatorname{Id}-K_{B}\right) \cdot \operatorname{det}\left(L_{B}\left(x_{i}, x_{j}\right)\right)_{1 \leq i, j \leq k}
$$

(It should be noted that (1.36) may be nonnegative even for non-Hermitian kernel $K$, it is easy to see that such $K$ still has nonnegative minors). It follows from the definition of $k$-point correlation functions that

$$
\rho_{k}\left(x_{1}, \ldots, x_{k}\right)=\sum_{j=1}^{\infty} \frac{1}{j !} \int_{B^{j}} p_{k+j}\left(x_{1}, \ldots, x_{k}, x_{k+1}, \ldots, x_{k+j}\right) d x_{k+1} \ldots d x_{k+j}
$$


The system of equations can be inversed :

$p_{k}\left(x_{1}, \ldots, x_{k}\right)=\sum_{j=0}^{\infty} \frac{(-1)^{j}}{j !} \int_{B^{j}} \rho_{k+j}\left(x_{1}, \ldots, x_{k}, x_{k+1}, \ldots, x_{k+j}\right) d x_{k+1} \cdots d x_{k+j}$

Functions $p_{k}\left(x_{1}, \ldots, x_{k}\right)$ are called Janossy probability densities (see [DVJ], p. 122) or exclusion probability densities (see [Ma]). It is easy to check that

$$
\sum_{j=0}^{\infty} \frac{1}{j !} \int_{B_{j}} p_{j}\left(x_{1}, \ldots, x_{j}\right) d x_{1} \ldots d x_{j}=1
$$

The r.h.s. of (1.36) still makes sense when $\left\|K_{B}\right\|=\lambda_{1}(B)=1$ (and therefore $p_{k}\left(x_{1}, \ldots, x_{k}\right)$ are properly defined in this case too $)$. Indeed, $\operatorname{det}\left(\operatorname{Id}-K_{B}\right)=$ $\prod_{j=1}^{\infty}\left(1-\lambda_{j}(B)\right)$ as a function of $\lambda_{1}$ has a zero of order 1 at $\lambda_{1}=1$. We claim that $\operatorname{det}\left(L\left(x_{i}, x_{j}\right)\right)_{1 \leq i, j \leq k}$ has a pole at $\lambda_{1}=1$ also of order 1 . To see this we write $L=\tilde{L}+\widetilde{\widetilde{L}}$, where $\tilde{L}_{i, j}=\frac{\lambda_{1}(B)}{1-\lambda_{1}(B)} \cdot \varphi_{1}\left(x_{i}\right) \cdot \overline{\varphi_{1}\left(x_{j}\right)}, \widetilde{\widetilde{L}}=$ $\sum_{\ell \geq 2} \frac{\lambda_{\ell}(B)}{1-\lambda_{\ell}(B)} \cdot \varphi_{\ell}\left(x_{i}\right) \cdot \overline{\varphi_{\ell}\left(x_{j}\right)}$. Then $\operatorname{det}\left(L\left(x_{i}, x_{j}\right)\right)_{1 \leq i, j \leq k}=\wedge^{k}\left(L\left(x_{i}, x_{j}\right)_{1 \leq i, j \leq k}\right)$, and we use the fact that $\operatorname{rank}(\tilde{L})=1$. If 1 is a multiple eigenvalue of $K \cdot \square_{B}$, say $\lambda_{1}(B)=\lambda_{2}(B)=\cdots=\lambda_{m}(B)=1>\lambda_{m+1}(B)$ one defines $\tilde{L}_{i, j}=\sum_{\ell=1}^{m} \frac{\lambda_{\ell}(B)}{1-\lambda_{\ell}(B)} \varphi_{\ell}\left(x_{i}\right) \cdot \overline{\varphi_{\ell}\left(x_{j}\right)}$ and proceeds in a similar manner.

Remark 5. Following Macchi, we call a random point field regular if for any Borel $B \subset E$ satisfying $\#_{B}<\infty$ (P-a.e.), the generating function $\mathbb{E} z^{\#_{B}}$ is entire. It follows from our results (see also Theorem 4 below) that any determinantal random point field is regular.

Remark 6. In [Ma] (Theorem 12, p. 113) (see also [DVJ], p. 138) Macchi essentially claimed that a necessary and sufficient condition on the integral operator $K$, locally trace class, to define a regular fermion (=determinantal in our notations) random point field is $0 \leq K<1$. As one can see from Theorem 3 above this condition is sufficient, but not necessary (as we established in Theorem 3, the necessary and sufficient condition is $0 \leq K \leq 1$ ). For completeness it should be noted that Macchi studied the case of continuous $K(x, y)$ with $\operatorname{Tr} K<\infty$.

Remark 7. Formula (1.36) was established in [Ma], p. 113 (see also [DVJ], p. 138 and [TW1], p. 820). 
We finish $\S 1$ with a few more results of general nature about determinantal random point fields.

\section{Theorem 4}

a) The probability of the event that the number of all particles is finite is either 0 or 1, depending on whether $\operatorname{Tr} K$ is finite or infinite.

b) The number of particles is less or equal to $n$ with probability 1 if and only if $K$ is a finite rank operator with rank $(K) \leq n$.

c) The number of particles is $n$ with probability 1 if and only if $K$ is an orthogonal projector with rank $(K)=n$.

d) For any determinantal random point field with probability 1 no two particles coincide.

e) To obtain results of the theorem for $B \subset E$ one has to replace $K$ by $K_{B}$.

\section{Proof of Theorem 4.}

a) One direction is obvious. Indeed, if $\operatorname{Tr} K=\mathbb{E} \#_{E}<+\infty$, then $\#_{E}<$ $+\infty$ with probability 1 . Let us now assume $\operatorname{Tr} K=+\infty$. Consider a monotone absorbing family of compact sets $\left\{B_{j}\right\}_{j=1}^{\infty}$ (i.e., $B_{i} \subset B_{i+1}$ and $\left.\bigcup_{i=1}^{\infty} B_{i}=E\right)$. Then $\operatorname{Tr} K_{B_{j}} \underset{j \rightarrow \infty}{\longrightarrow}+\infty$. Fix arbitrary large $N$. By the construction of $\left\{B_{j}\right\}$ we have $P\left(\#_{E} \leq N\right)=\lim _{j \rightarrow \infty} P\left(\#_{B_{j}} \leq N\right)$. But $P\left(\#_{B_{j}} \leq N\right) \leq 2^{N} \cdot \mathbb{E} 2^{-\#_{B_{j}}}=2^{N} \cdot \operatorname{det}\left(\operatorname{Id}-\frac{1}{2} \cdot K_{B_{j}}\right) \leq 2^{N}$. $e^{-\frac{1}{2} \operatorname{Tr}\left(K_{B_{j}}\right)} \underset{j \rightarrow \infty}{\longrightarrow} 0$.

b) If $\operatorname{rank}(K)=n$, then writing $K(x, y)=\sum_{i=1}^{n} \lambda_{i} \cdot \varphi_{i}(x) \cdot \overline{\varphi_{i}(y)}$ (a.e.), and $\rho_{n}\left(x_{1}, \ldots, x_{n}\right)=\operatorname{det}\left(K\left(x_{i}, x_{j}\right)\right)_{1 \leq i, j \leq n}$ we observe that $\rho_{m}\left(x_{1}, \ldots x_{m}\right)=$ 0 (a.e.) for any $m>n$. Therefore $\mathbb{E} \#_{E} \cdot\left(\#_{E}-1\right) \cdot \ldots \cdot\left(\#_{E}-n\right)=$ $\int \rho_{n+1}\left(x_{1}, \ldots x_{n+1}\right) d x_{1} \ldots d x_{n+1}=0$ which implies $\#_{E} \leq n$ with probability 1 .

In the opposite direction, if $\#_{E} \leq n$ (a.e.) we have $\int_{B^{n+1}} \rho_{n+1}\left(x_{1}, \ldots\right.$, $\left.x_{n+1}\right) d x_{1} \ldots d x_{n+1}=0$ for any bounded Borel $B \subset E$, therefore $\operatorname{Tr}\left(\wedge^{n+1}\right.$ $\left.\left(K_{B}\right)\right)=0$. Since $K \geq 0$ we obtain rank $\left(K_{B}\right) \leq n$ for arbitrary compact $B$, which implies rank $(K) \leq n$. 
c) follows from b) and the formula $\operatorname{Var}\left(\#_{E}\right)=\operatorname{Tr}\left(K-K^{2}\right)=\prod_{i=1}^{n} \lambda_{i}$. $\left(1-\lambda_{i}\right)$.

d) Let $B_{n}=[-n, n]^{d}$. It is enough to show that for any $n$ with probability 1 no two particles in $B_{n}$ coincide. Let $\epsilon$ be arbitrary small. Then $P\left\{\exists i \neq j: x_{i}=x_{j} \in B_{n}\right\} \leq P\left\{\exists i \neq j:\left|x_{i}-x_{j}\right|<\epsilon, x_{i} \in B_{n}, x_{j} \in\right.$ $\left.B_{n}\right\} \leq \int_{B_{n}}\left(\int_{|x-y|<\epsilon} \rho_{2}(x, y) d x\right) d y$. Since $\rho_{2}(x, y)$ is locally integrable, the last integral can be made arbitrary small by letting $\epsilon \rightarrow 0$.

The next result gives a criterion for the weak convergence of determinantal random point fields.

Theorem 5. Let $P$ and $P_{n}, n=1,2, \ldots$ be probability measures on $(X, B)$ corresponding to the determinantal random point fields defined by the Hermitian kernels $K$ and $K_{n}$. Let $K_{n}$ converge to $K$ in the weak operator topology and $\operatorname{Tr}\left(\chi_{B} K_{n} \chi_{B}\right) \underset{n \rightarrow \infty}{\longrightarrow} \operatorname{Tr}\left(\chi_{B} K \chi_{B}\right)$ for any bounded Borel $B \subset E$. Then the probability measures $P_{n}$ converge to $P$ weakly on the cylinder sets.

Proof of Theorem 5. It follows from [S1], Theorem 2.20, p. 40 that the assumptions of the theorem imply

$$
\operatorname{Tr}\left|\left(K_{n}-K\right)_{B}\right|=\left\|\left(K_{n}-K\right)_{B}\right\|_{1} \underset{n \rightarrow \infty}{\longrightarrow} 0 .
$$

As a consequence of (1.40) we have

$$
\operatorname{Tr} \cdot\left(K_{n} \cdot \chi_{B_{1}} \cdot \ldots \cdot K_{n} \cdot \chi_{B_{m}}\right) \underset{n \rightarrow \infty}{\longrightarrow} \operatorname{Tr}\left(K \cdot \chi_{B_{1}} \cdot \ldots \cdot K \cdot \chi_{B_{m}}\right)
$$

for any compact $B_{1}, \ldots, B_{m}$.

Thus using (1.26), (1.27) one can see that the joint moments of $\left\{\#_{B}\right\}$ with respect to $P_{n}$ converge to the joint moments with respect to $P$. Since the moments of $\#_{B}$ in the case of the determinantal random points define the distribution of $\#_{B}$ uniquely one can see (exercise) that $P_{n} \stackrel{W}{\longrightarrow} P$.

The rest of the notes is organized as follows. Section 2 is devoted to the various examples of determinantal random point fields arising in Quantum Mechanics, Statistical Mechanics, Random Matrix Theory, Representation Theory, Probability Theory (Renewal Process, 2D Random Growth Models). In $\S 3$ we discuss ergodic properties of the translation invariant determinantal random point fields. We also point out a special role played by the sine kernel $K(x, y)=\frac{\sin \pi(x-y)}{\pi(x-y)}$. In $\S 4$ we discuss the Central Limit Theorem for 
the counting measure and the Functional Central Limit Theorem for the empirical distribution function of spacings.

It is a great pleasure to thank Ya. Sinai for the encouragement to write this paper, B. Simon for the explaination of the result of Lemma 1 , G. Olshanski for many valuable remarks, and A. Borodin, B. Khoruzhenko, R. Killip, and Yu. Kondratiev for useful conversations.

\section{Examples of Determinantal Random Point Fields}

\subsection{Fermion Gas}

Let $H=-\frac{d^{2}}{d x^{2}}+V(x)$ be a Schrödinger operator with discrete spectrum acting on $L^{2}(E)$. Let $\left\{\varphi_{\ell}\right\}_{\ell=0}^{\infty}$ be an orthonormal basis of the eigenfunctions, $H \varphi_{\ell}=\lambda_{\ell} \cdot \varphi_{\ell}, \lambda_{0}<\lambda_{1} \leq \lambda_{2} \leq \ldots$. Consider the $n^{\text {th }}$ exterior power of $H, \wedge^{n}(H): \wedge^{n}\left(L^{2}(E)\right) \rightarrow \wedge^{n}\left(L^{2}(E)\right)$, where $\left.\wedge^{n}\left(L^{2}(E)\right)=A_{n} L^{2}\left(E^{n}\right)\right)$ is the space of square-integrable antisymmetric functions of $n$ variables and $\wedge^{n}(H)=\sum_{i=1}^{n}\left(-\frac{d^{2}}{d x_{i}^{2}}+V\left(x_{i}\right)\right)$. In Quantum Mechanics $\wedge^{n}(H)$ describes the Fermi gas with $n$ particles. The ground state of the Fermi gas is given by

$$
\begin{aligned}
& \psi\left(x_{1}, \ldots, x_{n}\right)= \\
& \frac{1}{\sqrt{n !}} \sum_{\sigma \in S_{n}}(-1)^{\sigma} \prod_{i=1}^{n} \varphi_{i-1}\left(x_{\sigma(i)}\right)=\frac{1}{\sqrt{n !}} \operatorname{det}\left(\varphi_{i-1}\left(x_{j}\right)\right)_{1 \leq i, j \leq n}
\end{aligned}
$$

It could be noted that $\psi\left(x_{1}, \ldots, x_{n}\right)$ coincides up to a sign $\epsilon\left(x_{1}, \ldots, x_{n}\right)$ with the ground state of $\sum_{i=1}^{n}\left(-\frac{d^{2}}{d x_{i}^{2}}+V\left(x_{i}\right)\right)$ acting on $S_{n} L^{2}\left(E^{n}\right)$ with the boundary conditions $\left.\psi\right|_{x_{i}=x_{j}}=0$. According to the postulate of Quantum Mechanics the absolute value squared of the ground state defines the probability distribution of $n$ particles. We write

$$
\begin{gathered}
p\left(x_{1}, \ldots, x_{n}\right)=\left|\psi\left(x_{1}, \ldots, x_{n}\right)\right|^{2}=\frac{1}{n !} \operatorname{det}\left(\varphi_{i-1}\left(x_{j}\right)\right)_{1 \leq i, j \leq n} \\
\cdot \operatorname{det}\left(\overline{\varphi_{j-1}\left(x_{i}\right)}\right)_{1 \leq i, j \leq n}=\frac{1}{n !} \operatorname{det}\left(K_{n}\left(x_{i}, x_{j}\right)\right)_{1 \leq i, j \leq n},
\end{gathered}
$$

where $K_{n}(x, y)=\sum_{i=0}^{n-1} \varphi_{i-1}(x) \overline{\varphi_{i-1}(y)}$ is the kernel of the orthogonal projector onto the subspace spanned by the first $n$ eigenfunctions of $H$. We claim 
that (2.2) defines a determinantal random point field. Indeed, the $k$-point correlation functions are given by

$$
\begin{aligned}
& \rho_{k}^{(n)}\left(x_{1}, \ldots, x_{n}\right)=\frac{n !}{(n-k) !} \int p_{n}\left(x_{1}, \ldots, x_{n}\right) d x_{k+1} \ldots d x_{n}= \\
& \quad \operatorname{det}\left(K_{n}\left(x_{1}, x_{j}\right)\right)_{1 \leq i, j \leq k}
\end{aligned}
$$

The last equality in (2.3) follows from the general lemma well known in Random Matrix Theory.

Lemma $4[\mathrm{Me}]$, p. 89). Let $(E, d \mu)$ be a measurable space and a kernel $K: E^{2} \rightarrow \mathbb{R}^{1}$ satisfy

$$
\begin{gathered}
\int_{E} K(x, y) \cdot K(y, z) d \mu(y)=K(x, z) \\
\int_{E} K(x, x) d \mu(x)=\mathrm{const}
\end{gathered}
$$

Then

$$
\begin{aligned}
& \int_{E} \operatorname{det}\left(K\left(x_{i}, x_{j}\right)\right)_{1 \leq i, j \leq n} d \mu\left(x_{n}\right)= \\
& \quad(\text { const }-n+1) \cdot \operatorname{det}\left(K\left(x_{i}, x_{j}\right)\right)_{1 \leq i, j \leq n-1}
\end{aligned}
$$

We shall consider in more detail two special cases of $H$. The first case is the harmonic oscillator

a) $H=-\frac{d^{2}}{d x^{2}}+x^{2}, E=\mathbb{R}^{1}$. Then

$$
\varphi_{\ell}(x)=\frac{(-1)^{\ell}}{\pi^{\frac{1}{4}} \cdot\left(2^{\ell} \cdot \ell !\right)^{\frac{1}{2}}} \exp \left(\frac{x^{2}}{2}\right) \frac{d^{\ell}}{d x^{\ell}}\left(\exp \left(-x^{2}\right)\right)
$$

are known as Weber-Hermite functions. To pass to the thermodynamic limit $n \rightarrow \infty$ we make a proper rescaling

$$
x_{i}=\frac{\pi}{(2 n)^{\frac{1}{2}}} y_{i}, i=1, \ldots, n .
$$


Then the Christoffel-Darboux formula and the Plancherel-Rotach asymptotics of the Hermite polynomials ([E]) imply that

$$
\begin{aligned}
& K_{n}\left(x_{1}, x_{2}\right)=\sum_{\ell=0}^{n-1} \varphi_{\ell}\left(x_{1}\right) \varphi_{\ell}\left(x_{2}\right)= \\
& \left(\frac{n}{2}\right)^{\frac{1}{2}}\left[\frac{\varphi_{n}\left(x_{1}\right) \cdot \varphi_{n-1}\left(x_{2}\right)-\varphi_{n}\left(x_{2}\right) \varphi_{n-1}\left(x_{1}\right)}{x_{1}-x_{2}}\right]
\end{aligned}
$$

has a limit as $n \rightarrow+\infty$

$$
K_{n}\left(x_{1}, x_{2}\right) \underset{n \rightarrow \infty}{\longrightarrow} K\left(y_{1}, y_{2}\right)=\frac{\sin \pi\left(y_{1}-y_{2}\right)}{\pi\left(y_{1}-y_{2}\right)}
$$

The convergence of kernels implies the convergence of $k$-point correlation functions, which in turn implies the weak convergence of the distribution

$$
\left(\frac{\pi}{(2 n)^{\frac{1}{2}}}\right)^{n} p_{n}\left(\frac{\pi}{(2 n)^{\frac{1}{2}}} y_{1}, \ldots, \frac{\pi}{(2 n)^{\frac{1}{2}}} y_{n}\right) d y_{1} \ldots d y_{n}
$$

to the translation-invariant determinantal random point field with the "sine kernel" $K\left(y_{1}, y_{2}\right)=\frac{\sin \pi\left(y_{1}-y_{2}\right)}{\pi\left(y_{1}-y_{2}\right)}$.

b) For another example let $E=S^{1}=\left\{z=e^{i \theta}, 0 \leq \theta<2 \pi\right\}, H=-\frac{d^{2}}{d \theta^{2}}$. Then

$$
\begin{aligned}
& \varphi_{\ell}(\theta)=\frac{1}{\sqrt{2 \pi}} e^{i \ell \theta}, \\
& p_{n}\left(\theta_{1}, \ldots, \theta_{n}\right)=\frac{1}{n !} \operatorname{det}\left(\sum_{\ell=0}^{n-1} \frac{1}{2 \pi} e^{i \ell\left(\theta_{j}-\theta_{k}\right)}\right)_{1 \leq j, k \leq n}= \\
& \frac{1}{n !} \operatorname{det}\left(K_{n}\left(\theta_{i}, \theta_{j}\right)_{1 \leq i, j \leq n}\right.
\end{aligned}
$$

where

$$
K_{n}\left(\theta_{1}, \theta_{2}\right)=\frac{1}{2 \pi} \frac{\sin \left(\frac{n}{2} \cdot\left(\theta_{2}-\theta_{1}\right)\right)}{\sin \left(\frac{\theta_{2}-\theta_{1}}{2}\right)}
$$

After rescaling $\frac{n}{2 \pi} \theta_{i}=y_{i}, i=1, \ldots, n$ the rescaled correlation functions have the same limit as in (2.9), in particular

$$
\lim _{n \rightarrow \infty} \frac{2 \pi}{n} K_{n}\left(\frac{2 \pi}{n} y_{1}, \frac{2 \pi}{n} y_{2}\right)=\frac{\sin \pi\left(y_{2}-y_{1}\right)}{\pi\left(y_{2}-y_{1}\right)} .
$$


For more information we refer the reader to [D1]-[D3], [L4]-[L5], [Sp].

\subsection{Coulomb Gas at $\beta=2$}

Examples a), b) from $\S 2.1$ can be reinterpreted as the equilibrium distribution of $n$ unit charges confined to the one-dimensional line (ex. 2.1a)) or the unit circle (ex. 2.1b)) repelling each other according to the Coulomb law of twodimensional electrostatics. Writing the potential energy as $H\left(z_{1}, \ldots, z_{n}\right)=$

$-\sum_{1 \leq i<j \leq n} \log \left|z_{i}-z_{j}\right|+\sum_{i=1}^{n} V\left(z_{i}\right)$, where $V$ is an external potential, we note that the Boltzmann factor $\frac{1}{Z} \exp \left(-\beta H\left(z_{1}, \ldots, z_{n}\right)\right), \beta=2$, is exactly $p_{n}\left(z_{1}, \ldots z_{n}\right)$ in 2.1a) with $V(z)=\frac{1}{2} z^{2}$, and $p_{n}\left(\theta_{1}, \ldots, \theta_{n}\right)$ in $\left.\S 2.1 \mathrm{~b}\right)$ with $V(z)=0, z_{j}=e^{i \theta_{j}}, j=1, \ldots, n$.

The one-component Coulomb gas in two dimensions (a.k.a. a two-dimensional one-component plasma) was studied in a number of papers including [Gin], [Ja1], [Ja2], [AL], [DFGIL], [FJ1]. This subject is closely related to the theory of non-Hermitian Gaussian random matrices (to be discussed in $\S 2.3 \mathrm{~d}$ ). The two-component two-dimensional Coulomb gas (i.e. a system of positively and negatively charged particles) was studied in [Ga], [CJ1]-[CJ3], [AF], [FJ2]. Let us start with a neutral system of $n$ positive and $n$ negative particles. After denoting the complex coordinates by $u_{j}$ and $v_{j}, j=1, \ldots, n$, we write the Boltzmann factor at $\beta=2$ as

$$
\begin{gathered}
\exp \left(2 \sum_{1 \leq i<j \leq n}\left(\log \left|u_{i}-u_{j}\right|+\log \left|v_{i}-v_{j}\right|-2 \log \left|u_{i}-v_{j}\right|\right)\right)= \\
\frac{\prod_{1 \leq i<j \leq n}\left|u_{i}-u_{j}\right|^{2} \cdot\left|v_{i}-v_{j}\right|^{2}}{\prod_{i, j}\left|u_{i}-v_{j}\right|^{2}}=\left|\operatorname{det}\left(\frac{1}{u_{i}-v_{j}}\right)_{1 \leq i, j \leq n}\right|^{2} .
\end{gathered}
$$

Discretizing the model one allows the positive particles to occupy only the sites of the sublattice $\gamma \cdot \mathbb{Z}^{2}$ and the negative particles to occupy only the sites of the sublattice $\gamma \cdot\left(\mathbb{Z}^{2}+\left(\frac{1}{2}, \frac{1}{2}\right)\right)$. The grand canonical ensemble is defined by the partition function (let $\gamma=1$ )

$$
\begin{aligned}
Z= & 1+\sum_{u, v} \lambda_{+}(u) \lambda_{-}(v) \cdot \frac{1}{|u-v|^{2}}+\left(\frac{1}{2 !}\right)^{2} \\
& \sum_{u_{1}, u_{2}, v_{1}, v_{2}} \lambda_{+}\left(u_{1}\right) \lambda_{+}\left(u_{2}\right) \lambda_{+}\left(v_{1}\right) \lambda_{+}\left(v_{2}\right) \cdot\left|\operatorname{det}\left(\frac{1}{u_{i}-v_{j}}\right)_{1 \leq i, j \leq 2}\right|^{2}+\cdots,
\end{aligned}
$$


where $\lambda_{+}(u)=e^{-V(u)}, \lambda_{-}(u)=e^{V(u)}$, are fugacities and $V$ is an external potential. One can rewrite the last formula as

$$
\begin{aligned}
Z= & \operatorname{det}\left(\operatorname{Id}+\left(\lambda_{+} \frac{1+\sigma_{z}}{2}+\lambda_{-} \frac{1-\sigma_{z}}{2}\right)\right. \\
& \left.\cdot\left(\frac{\sigma_{x}+i \sigma_{y}}{2} \cdot \frac{1}{z-z^{\prime}}+\frac{\sigma_{x}-i \sigma_{y}}{2} \cdot \frac{1}{\bar{z}-\overline{z^{\prime}}}\right)\right),
\end{aligned}
$$

where $\sigma_{x}, \sigma_{y}, \sigma_{z}$ are $2 \times 2$ Pauli matrices. In particular we see that the grand canonical ensemble is a discrete fermion random point field (the appearance of matrix-valued kernel reflects the fact that $\left.E=\mathbb{Z}^{2} \bigsqcup\left(\mathbb{Z}^{2}+\left(\frac{1}{2}, \frac{1}{2}\right)\right)\right)$. Passing to the continuous limit $(\gamma=0)$ one can see that two- and higher order correlation functions have a limit, and the limiting kernel $K$ can be expressed in terms of the Green function of a differential Dirac operator, namely

$$
\begin{aligned}
K= & \left(m_{+} \cdot \frac{1+\sigma_{z}}{2}+m_{-} \cdot \frac{1-\sigma_{z}}{2}\right) \\
& \left(\sigma_{x} \partial_{x}+\sigma_{y} \partial_{y}+m_{+} \cdot \frac{1+\sigma_{z}}{2}+m_{-} \frac{1-\sigma_{z}}{2}\right)^{-1},
\end{aligned}
$$

where $m_{+}, m_{-}$are rescaled fugacities. In the special case $m_{+}=m_{-} \equiv$ const

(i.e., $V \equiv 0), K=\left(\begin{array}{ll}K++, & K+- \\ K-+, & K--\end{array}\right)$ can be expressed in terms of modified Bessel function (for the details see e.g., [CJ3]).

\subsection{Random Matrix Models}

a) Unitary Invariant Ensembles of Hermitian Randon Matrices

The probability distribution in §2.1a) (formulas (2.2), (2.7)) allows yet another interpretation. It is well known in Random Matrix Theory as the distribution of the eigenvalues in the Gaussian Unitary Ensemble (G.U.E.). We recall the definition of G.U.E. Consider the space of $n \times n$ Hermitian matrices $\left\{A=\left(A_{i j}\right)_{1 \leq i, j \leq n}, \operatorname{Re}\left(A_{i j}\right)=\operatorname{Re}\left(A_{j i}\right), \operatorname{Im}\left(A_{i j}\right)=-\operatorname{Im}\left(A_{j i}\right)\right\} . \quad \mathrm{A}$ G.U.E. random matrix is defined by its probability distribution

$$
P(d A)=\text { const }_{n} \cdot \exp \left(-\operatorname{Tr} A^{2}\right) d A,
$$

where $d A$ is a flat (Lebesgue) measure, i.e., $d A=\prod_{i<j} d \operatorname{Re}\left(A_{i j}\right)$ $d \operatorname{Im}\left(A_{i j}\right) \prod_{k=1}^{n} d A_{k k}$. The definition of G.U.E. is equivalent to the requirement that $\left\{\operatorname{Re}\left(A_{i j}\right), \operatorname{Im}\left(A_{i j}\right), 1 \leq i<j \leq n, A_{k k}, 1 \leq k \leq n\right\}$ are mutually 
independent and $\operatorname{Re}\left(A_{i j}\right) \sim N\left(0, \frac{1}{4}\right), \operatorname{Im}\left(A_{i j}\right) \sim N\left(0, \frac{1}{4}\right), A_{k k} \sim N\left(0, \frac{1}{2}\right)$. The eigenvalues of a random Hermitian matrix are real random variables. For the derivation of their joint distribution we refer the reader to [De], sections 5.3-5.4 and [Me], chapters 3, 5. It appears that the density of the joint distribution with respect to the Lebesgue measure is given exactly by (2.2), (2.7).

We remark that the distribution of a G.U.E. random matrix is invariant under the unitary transformation $A \rightarrow U A U^{-1}, U \in U(n)$. A natural generalization of (2.12) that preserves the unitary invariance is

$$
P(d A)=\text { const }_{n} \cdot \exp (-2 \cdot \operatorname{Tr} V(A)) d A
$$

where $V(x)$ can be, for example, a polynomial of even degree with a positive leading coefficients (see [De], section 5). The derivation of the formula for the joint distribution of the eigenvalues is very similar to the G.U.E. case. The density $p_{n}\left(\lambda_{1}, \ldots, \lambda_{n}\right)$ is given by $(2.2)$, where $\left\{\varphi_{\ell}(x) \cdot e^{V(x)}\right\}_{\ell=0}^{n-1}$ are the first $n$ orthonormal polynomials with respect to the weight $\exp (-2 V(x)) . K_{n}(x, y)$ is then again a kernel of a projector and therefore satisfies the conditions of Lemma 4.

b) Random Unitary Matrices

Let us consider the group of $n \times n$ unitary matrices $U(n)$. There exists a unique translation invariant probability measure on $U(n)$ (see [We]). It is called the Haar measure, we will denote it by $\mu_{\text {Haar }}$. The probability density of the induced distribution of the eigenvalues is given by

$$
p_{n}\left(\theta_{1}, \ldots, \theta_{n}\right)=(2 \pi)^{-n} \cdot \frac{1}{n !} \cdot \prod_{1 \leq k<\ell \leq n}\left|e^{i \theta_{k}}-e^{i \theta_{\ell}}\right|^{2},
$$

which coincides with (2.10)-(2.11) (see [Me], ch. 9-10, [D1]-[D3]). In the last formula we used the notations

$$
\lambda_{1}=e^{i \theta_{1}}, \ldots, \lambda_{n}=e^{i \theta_{n}} .
$$

If one starts with the probability measure const $_{n} \cdot e^{-\operatorname{Tr} V(U)} d \mu_{\text {Haar }}(U)$ on the unitary group instead of the Haar measure, and replaces the monomials $\frac{1}{\sqrt{2 \pi}} e^{i \ell \theta}$ by $\psi_{\ell}(\theta) \cdot e^{-\frac{1}{2} V(\theta)}$, where $\left\{\psi_{\ell}\right\}_{\ell=0}^{n-1}$ are the first $n$ orthonormal polynomials in $e^{i \theta}$ with respect to the weight $e^{-V(\theta)} d \theta$, one stills arrives at the formula (2.10) for the $k$-point correlation functions. 
c) Random Orthogonal and Symplectic Matrices

The distribution of the eigenvalues of a random orthogonal or symplectic matrix (with respect to the Haar measure) also has a form of a determinantial random point field with a fixed number of particles. For the convenience of the reader we draw below the chart of the kernels appearing in the ensembles of random matrices from the Classical Compact Groups.

\begin{tabular}{|l|l|}
\hline & $K_{n}(x, y)$ \\
\hline$U(n)$ & $\frac{1}{2 \pi} \cdot \frac{\sin \left(\frac{n}{2} \cdot(x-y)\right)}{\sin \left(\frac{x-y}{2}\right)} ; E=[0,2 \pi]$ \\
\hline$S O(2 n)$ & $\frac{1}{2 \pi} \cdot\left(\frac{\sin \left(\frac{2 n-1}{2} \cdot(x-y)\right)}{\sin \left(\frac{x-y}{2}\right)}+\frac{\sin \left(\frac{2 n-1}{2} \cdot(x+y)\right)}{\sin \left(\frac{x+y}{2}\right)}\right) ; E=[0, \pi]$ \\
\hline$S O(2 n+1)$ & $\frac{1}{2 \pi} \cdot\left(\frac{\sin (n \cdot(x-y))}{\sin \left(\frac{x-y}{2}\right)}-\frac{\sin (n \cdot(x+y))}{\sin \left(\frac{x+y}{2}\right)}\right) ; E=[0, \pi]$ \\
\hline$S p(n)$ & $\frac{1}{2 \pi}\left(\frac{\sin \left(\frac{2 n+1}{2} \cdot(x-y)\right)}{\sin \left(\frac{x-y}{2}\right)}-\frac{\sin \left(\frac{2 n+1}{2} \cdot(x+y)\right)}{\sin \left(\frac{x+y}{2}\right)}\right) ; E=[0, \pi]$ \\
\hline $0 \_(2 n+2)$ & the same as for $S p(n)$ \\
\hline
\end{tabular}

For additional information we refer the reader to [Jo1], [DS], [KS], [So1], [So2], [So3].

d) Complex Non-Hermitian Gaussian Random Matrices

In [Gin] Ginibre considered the ensemble of complex non-Hermitian random $n \times n$ matrices where all $2 n^{2}$ parameters $\left\{\operatorname{Re} A_{i j}, \operatorname{Im} A_{i j}, 1 \leq i, j \leq n\right\}$ are independent Gaussian random variables with zero mean and variance $\frac{1}{2}$. The joint probability distribution of the matrix elements is then given by the formula

$$
\begin{aligned}
& P(d A)=\text { const }_{n} \cdot \exp \left(-\operatorname{Tr}\left(A^{*} \cdot A\right)\right) d A, \\
& d A=\prod_{1 \leq j, k \leq n} d \operatorname{Re} A_{j k} \cdot d \operatorname{Im} A_{j k} .
\end{aligned}
$$

The equivalent definition of (2.14) is that $A=\tilde{A}+i \cdot \widetilde{\widetilde{A}}$, where $\tilde{A}$ and $\widetilde{\widetilde{A}}$ are two independent G.U.E. matrices. The eigenvalues $\lambda_{1}, \ldots, \lambda_{n}$ are complex 
random variables. It was shown that their distribution is given by the determinantal random point field in $\mathbb{R}^{2}$ with a fixed number of particles $(\#=n)$ and the correlation functions

$$
\rho_{k}^{(n)}\left(z_{1}, \ldots, z_{k}\right)=\operatorname{det}\left(K_{n}\left(z_{j}, \overline{z_{m}}\right)\right)_{1 \leq j, m \leq n}
$$

where $K_{n}\left(z_{1}, \overline{z_{2}}\right)=\frac{1}{\pi} \exp \left(-\frac{\left|z_{1}\right|^{2}}{2}-\frac{\left|z_{2}\right|^{2}}{2}\right) \cdot \sum_{\ell=0}^{n-1} \frac{z_{1}^{\ell} \overline{z_{2}} \ell}{\ell !}$. We mention in passing that $K_{n}\left(z_{1}, \overline{z_{2}}\right)$ converges to the kernel

$$
K\left(z_{1}, \overline{z_{2}}\right)=\frac{1}{\pi} \exp \left(-\frac{\left|z_{1}\right|^{2}}{2}-\frac{\left|z_{2}\right|^{2}}{2}+z_{1} \cdot \overline{z_{2}}\right)
$$

which defines the limiting random point field. A generalization of (2.14) was studied in [Gir1], [Gir2], [SCSS], [FKS1], [FKS2]. Let $A=\tilde{A}+i \cdot v \cdot \tilde{\widetilde{A}}$, where $\tilde{A}$ and $\tilde{\widetilde{A}}$ are, as above, two independent G.U.E. matrices and $v$ is a real parameter (it is enough to consider $0 \leq v \leq 1$ ). Let us introduce a new parameter $\tau=\frac{1-v^{2}}{1+v^{2}}$. The distribution of the matrix elements is given by

$$
P(d A)=\text { const }_{n} \cdot \exp \left(-\frac{1}{1-\tau^{2}} \operatorname{Tr}\left(A^{*} A-\tau \operatorname{Re}\left(A^{2}\right)\right)\right) d A
$$

It induces the distribution of the eigenvalues

$$
\begin{aligned}
& p_{n}\left(z_{1}, \ldots, z_{n}\right) \prod_{j=1}^{n} d z_{j} d \overline{z_{j}}=\mathrm{const}_{n} \cdot \exp \left[-\frac{1}{1-\tau^{2}} \cdot \sum_{j=1}^{n}\right. \\
& \left.\left(\left|z_{j}\right|^{2}-\frac{\tau}{2}\left(z_{j}^{2}+{\overline{z_{j}}}^{2}\right)\right)\right] \cdot \prod_{j<k}\left|z_{j}-z_{k}\right|^{2} \cdot \prod_{j=1}^{n} d z_{j} d \overline{z_{j}}
\end{aligned}
$$

It should be noted that the expression (2.18) also appeared in the papers by DiFranceso et al. ([DFGIL]) and Forrester-Jancovici ([FJ1]) as the Boltzmann factor of the two-dimensional one-component plasma. For the calculation of the correlation functions we refer the reader to [DFGIL], [FJ1], [FKS1], [FKS2]. The crucial role there is played by the orthonormal polynomials in the complex plane with the weight

$$
w^{2}(z)=\exp \left[-\frac{1}{1-\tau^{2}}\left(|z|^{2}-\frac{\tau}{2}\left(z^{2}+\bar{z}^{2}\right)\right)\right]
$$


Such orthonormal polynomials can be expressed in terms of the Hermite polynomials,

$$
\psi_{\ell}(z)=\frac{\tau^{\frac{\ell}{2}}}{\pi^{\frac{1}{2}} \cdot(\ell !)^{\frac{1}{2}} \cdot\left(1-\tau^{2}\right)^{\frac{1}{4}}} H_{\ell}\left(\frac{z}{\sqrt{\tau}}\right), \ell=0,1, \ldots
$$

where $\sum_{n=0}^{\infty} H_{n}(z) \cdot \frac{t^{n}}{n !}=\exp \left(z t-\frac{t^{2}}{2}\right)$. We remark that if $\tau=0$ (Ginibre case) $\psi_{\ell}(z)=\frac{1}{\pi^{\frac{1}{2}} \cdot(\ell !)^{\frac{1}{2}}} \cdot z^{\ell}$. Formula for the correlation functions ( $\tau$ arbitrary) generalzies $(2.15)$ :

$$
\begin{array}{r}
\rho_{k}^{(n)}=\operatorname{det}\left(K_{n}\left(z_{i}, \overline{z_{j}}\right)\right)_{1 \leq i, j \leq k}, \\
K_{n}\left(z_{1}, \overline{z_{2}}\right)=w\left(z_{1}\right) w\left(\overline{z_{2}}\right) \cdot \sum_{\ell=0}^{n-1} \psi_{\ell}\left(z_{1}\right) \psi_{\ell}\left(\overline{z_{2}}\right)
\end{array}
$$

In the limit $n \rightarrow \infty K_{n}\left(z, \overline{z_{2}}\right)$ converges to

$$
\begin{aligned}
& K\left(z_{1}, \overline{z_{2}}\right)=\lim _{n \rightarrow \infty} K_{n}\left(z_{1}, \overline{z_{2}}\right)= \\
& \quad \frac{1}{\pi\left(1-\tau^{2}\right)} \exp \left(-\frac{1}{1-\tau^{2}}\left(\frac{\left|z_{1}\right|^{2}}{2}+\frac{\left|z_{2}\right|^{2}}{2}-z_{1} \overline{z_{2}}\right)\right)
\end{aligned}
$$

We reamrk that the last formula differs from (2.16) only by the trivial rescaling $z \rightarrow z \cdot \sqrt{1-\tau^{2}}$. A special regime, called the regime of weak nonHermiticity, was discovered for the model (2.17) by Fyodorov, Khoruzhenko and Sommers in [FKS1], [FKS2]. Let

$$
\begin{aligned}
& \operatorname{Re}\left(z_{1}\right)=n^{\frac{1}{2}} \cdot x+n^{-\frac{1}{2}} x_{1}, \\
& \operatorname{Re}\left(z_{2}\right)=n^{\frac{1}{2}} \cdot x+n^{-\frac{1}{2}} x_{2}, \\
& \operatorname{Im}\left(z_{1}\right)=n^{-\frac{1}{2}} \cdot y_{1}, \\
& \operatorname{Im}\left(z_{2}\right)=n^{-\frac{1}{2}} \cdot y_{2},
\end{aligned}
$$

Assume the parameters $x, x_{1}, x_{2}, y_{1}, y_{2}$ fixed and take the limit $n \rightarrow \infty$ in such a way that $\lim _{n \rightarrow \infty} n \cdot(1-\tau)=\frac{\alpha^{2}}{2}$. Then

$$
\begin{aligned}
& \lim _{n \rightarrow \infty} \frac{1}{n} K_{n}\left(z_{1}, z_{2}\right)=\frac{1}{\pi \alpha} . \\
& \exp \left[-\frac{y_{1}^{2}+y_{2}^{2}}{\alpha^{2}}+i \cdot x \cdot \frac{\left(y_{1}-y_{2}\right)}{2}\right] \cdot g_{\alpha}\left(\frac{y_{1}+y_{2}}{2}-i \cdot \frac{\left(x_{1}-x_{2}\right)}{2}\right),
\end{aligned}
$$


where

$$
g_{\alpha}(y)=\int_{-\sqrt{1-\frac{x^{2}}{4}}}^{\sqrt{1-\frac{x^{2}}{4}}} \frac{d u}{\sqrt{2 \pi}} \exp \left[-\frac{\alpha^{2} u^{2}}{2}-2 u y\right] .
$$

(if $x>2$ the limit in (2.23) is equal to zero). The formulas $(2.23),(2.24)$ define determinantal random point field in $\mathbb{R}^{2}$, different from (2.16).

e) Positive Hermitian Random Matrices

Following Bronk $[\mathrm{Br}]$ we define the Laguerre ensemble of positive Hermitian $n \times n$ matrices. Any positive Hermitian matrix $M$ can be written as $M=A^{*} A$, where $A$ is some complex matrix. The probability distribution of a random matrix $M$ is given by

$$
\text { const }_{n} \cdot \exp \left(-\operatorname{Tr} A^{*} A\right) \cdot\left[\operatorname{det}\left(A^{*} A\right)\right]^{\alpha} d A
$$

where $d A$ is defined as in (2.14) and $\alpha>-1$ (the values of $\alpha$ of special interest are $\left.\pm \frac{1}{2}, 0\right)$. The induced probability distribution of the (positive) eigenvalues is given by

$$
\text { const }_{n} \cdot \exp \left(-\sum_{i=1}^{n} \lambda_{i}\right) \cdot \prod_{i=1}^{n} \lambda_{i}^{\alpha} \cdot \prod_{1 \leq i<j \leq n}\left(\lambda_{i}-\lambda_{j}\right)^{2} d \lambda_{1} \ldots \lambda_{n}
$$

Employing the associated Laguerre polynominals

$$
L_{m}^{\alpha}(x) \equiv \frac{1}{n !} e^{x} x^{-\alpha} \frac{d^{m}}{d x^{m}}\left(e^{-x} x^{m+\alpha}\right), m=0,1, \ldots
$$

one can rewrite $(2.26)$ as

$$
\frac{1}{n !} \operatorname{det}\left(K_{n}\left(x_{i}, x_{j}\right)\right)_{1 \leq i, j \leq n}
$$

where

$$
K_{n}(x, y)=\sum_{\ell=0}^{n-1} \varphi_{\ell}^{(\alpha)}(x) \cdot \varphi_{\ell}^{(\alpha)}(y)
$$

and $\left\{\varphi_{\ell}^{(\alpha)}(x)=\left(\Gamma(\alpha+1) \cdot\left(\begin{array}{c}n+\alpha \\ n\end{array}\right)\right)^{-\frac{1}{2}} L_{\ell}^{\alpha}(x)\right\}_{\ell=0}^{\infty}$ is the orthonormal basis with respect to the weight $e^{-x} \cdot x^{\alpha}$ on the positive semiaxis. Once again Lemma 4 
allows us to calculate explicitly $k$-point correlation functions and show that they are given by determinants of $k \times k$ matrices with the kernel (2.28).

f) Hermitian Matrices Coupled in a Chain

Let $A_{1}, \ldots, A_{p}$ be complex hermitian random $n \times n$ matrices with the joint probability density

$$
\begin{aligned}
& \text { const }_{n} \cdot \exp \left[-\operatorname{Tr}\left(\frac{1}{2} V_{1}\left(A_{1}\right)+V_{2}\left(A_{2}\right)+\cdots+V_{p-1}\left(A_{p-1}\right)+\frac{1}{2} V_{p}\left(A_{p}\right)\right.\right. \\
& \left.\left.+c_{1} A_{1} A_{2}+c_{2} A_{2} A_{3}+\cdots+c_{p-1} A_{p-1} A_{p}\right)\right]
\end{aligned}
$$

We denote the eigenvalues of $A_{j}$ (all real) by $\widetilde{\lambda_{j}}=\left(\lambda_{j 1}, \ldots, \lambda_{j n}\right), j=1, \ldots, p$. The induced probability densit of the eigenvalues is then equal to

$$
\begin{gathered}
P_{n}\left(\tilde{\lambda_{1}}, \ldots, \tilde{\lambda_{p}}\right)=\text { const }_{n} \cdot\left[\prod_{1 \leq r<s \leq n}\left(\lambda_{1 r}-\lambda_{1 s}\right)\left(\lambda_{p r}-\lambda_{p s}\right)\right] . \\
{\left[\prod_{k=1}^{p-1} \operatorname{det}\left[w_{k}\left(\lambda_{k r}, \lambda_{k+1 s}\right)\right]_{r, s=1, \ldots, n}\right]}
\end{gathered}
$$

where

$$
w_{k}(x, y)=\exp \left(-\frac{1}{2} V_{k}(x)-\frac{1}{2} V_{k+1}(y)+c_{k} x y\right)
$$

Eynard and Mehta [EM] established that the correlation functions of this model $\rho_{k_{1}, \ldots, k_{p}}\left(\lambda_{11}, \ldots, \lambda_{1 k_{1}} ; \ldots ; \lambda_{p 1}, \ldots, \lambda_{p k_{p}}\right)=\prod_{j=1}^{p} \frac{n !}{\left(n-k_{j}\right) !} \int p_{n}\left(\widetilde{\lambda_{1}}, \ldots, \tilde{\lambda_{p}}\right)$. $\prod_{j=1}^{p} \prod_{r_{j}=k_{j}+1}^{n} d \lambda_{j r_{j}}$ can be written as a $k \times k$ determinant with $k=k_{1}+$ $\cdots+k_{p}$

$$
\operatorname{det}\left[K_{i j}\left(\lambda_{i r}, \lambda_{j s}\right)\right]_{r=1, \ldots, k_{i} ; s=1, \ldots k_{j} ; i, j=1, \ldots, p}
$$

For the exact formulas for the kernels $K_{i j}(x, y)$ we refer the reader to [EM] (see also $[\mathrm{AM}]$ ). We remark that (2.32) defines a determinantal random point field with one-particle space $E$ being the union of $p$ copies of $\mathbb{R}^{1}$.

g) Universality in Random Matrix Models. Airy, Bessel and sine Random Point Fields. 
We start with a general class of kernels of the form

$$
K(x, y)=\frac{\varphi(x) \cdot \psi(y)-\varphi(y) \psi(x)}{x-y}
$$

where

$$
\begin{aligned}
& m(x) \varphi^{\prime}(x)=A(x) \varphi(x)+B(x) \psi(x) \\
& m(x) \psi^{\prime}(x)=-C(x) \varphi(x)-A(x) \psi(x)
\end{aligned}
$$

and $m(x), A(x), B(x), C(x)$ are polynomials. It was shown by Tracy and Widom ([TW2]) that Fredholm determinants of integral operators with kernels (2.33)-(2.34) restricted to a finite union of intervals satisfy certain partial differential equations. Airy, Bessel and sine kernels are the special cases of (2.33), (2.34). To define sine kernel we set $\varphi(x) \equiv \frac{1}{\pi} \sin (\pi x), \psi(x) \equiv$ $\varphi^{\prime}(x)\left(m(x) \equiv 1, A(x) \equiv 0, B(x) \equiv 1, C(x) \equiv \pi^{2}\right)$. For the Airy kernel $\varphi(x) \equiv A_{i}(x), \psi(x) \equiv \varphi^{\prime}(x)(m(x) \equiv 1, A(x) \equiv 0, B(x) \equiv 1, C(x) \equiv-x)$. For the Bessel kernel $\varphi(x) \equiv J_{\alpha}(\sqrt{x}), \psi(x) \equiv x \varphi^{\prime}(x)(m(x) \equiv x, A(x) \equiv$ $\left.0, B(x) \equiv 1, C(x) \equiv \frac{1}{4}\left(x-\alpha^{2}\right)\right)$. Here $A_{i}(x)$ is the Airy function and $J_{\alpha}(x)$ is the Bessel function of order $\alpha$ (see [E]). Writing down these kernels explicitly we have (see [TW2], [TW3], [TW4])

$$
\begin{gathered}
K_{\text {sine }}(x, y)=\frac{\sin \pi(x-y)}{\pi(x-y)}, \\
K_{\text {Airy }}(x, y)=\frac{\mathcal{A}_{i}(x) \cdot \mathcal{A}_{i}^{\prime}(y)-\mathcal{A}_{i}(y) \mathcal{A}_{i}^{\prime}(x)}{x-y} \\
=\int_{0}^{\infty} A_{i}(x+t) \mathcal{A}_{i}(y+t) d t \\
K_{\text {Bessel }}(x, y)=\frac{J_{\alpha}(\sqrt{x}) \cdot \sqrt{y} \cdot J_{\alpha}^{\prime}(\sqrt{y})-\sqrt{x} J_{\alpha}^{\prime}(\sqrt{x}) \cdot J_{\alpha}(\sqrt{y})}{2 \cdot(x-y)} \\
=\frac{\sqrt{x} \cdot J_{\alpha+1}(\sqrt{x}) \cdot J_{\alpha}(\sqrt{y})-J_{\alpha}(\sqrt{x}) \sqrt{y} \cdot J_{\alpha+1}(\sqrt{y})}{2 \cdot(x-y)}
\end{gathered}
$$

As we already mentioned, sine kernel appears as a scaling limit in the bulk of the spectrum in G.U.E. ([Me], Chapter 5). In its turn, Airy kernel appears as a scaling limit at the edge of the spectrum in G.U.E. and at the (soft) right 
edge of the spectrum in the Laguerre ensemble, while Bessel kernel appears as a scaling limit at the (hard) left edge in the Laguerre ensemble ([F], [TW3], [TW4]). Universality conjecture in Random Matrix Theory asserts that such limits should be universal for a wide class of Hermitian random matrices. Recently this conjecture was proven for unitary invariant ensembles (2.13) in the bulk of the spectrum ([PS], [BI], [DKMLVZ], [De]) and for some classes of Wigner matrices in the bulk of the spectrum [Jo4] and at the edge [So4].

In the next subsection we completely characterize determinantal random point fields in $\mathbb{R}^{1}\left(\mathbb{Z}^{1}\right)$ with independent identically distributed spacings.

\subsection{Determinantal random point fields with i.i.d. spac- ings. Renewal processes}

We start with some basic facts from the theory of renewal processes (see e.g., $[\mathrm{Fe}],[\mathrm{DVJ}])$. Let $\left\{\tau_{k}\right\}_{k=1}^{\infty}$ be independent identically distributed non-negative random variables and $\tau_{0}$ another non-negative random variable independent from $\left\{\tau_{k}\right\}_{k=1}^{\infty}$ (in general the distribution of $\tau_{0}$ will be different). We define

$$
x_{k}=\sum_{j=0}^{k} \tau_{j} .
$$

This gives us a random configuration $\left\{x_{k}\right\}_{k=0}^{\infty}$ in $\mathbb{R}_{+}^{1}$. In probability theory a random sequence $\left\{x_{k}\right\}_{k=0}^{\infty}$ is known as delayed renewal process. We assume that the distribution of random variables $\tau_{k}, k \geq 1$ has density $f(x)$, called interval distribution density, and a finite mathematical expectation $E \tau_{1}=$ $\int_{0}^{\infty} x f(x) d x$. The renewal density is defined than as

$$
\begin{aligned}
u(x)= & \sum_{k=1}^{\infty} f^{k *}(x)=f(x)+\int_{0}^{x} f(x-y) f(y) d y+ \\
& \int_{0}^{x} \int_{0}^{x-y_{2}} f\left(x-y_{1}-y_{2}\right) f\left(y_{1}\right) f\left(y_{2}\right) d y_{1} d y_{2}+\ldots
\end{aligned}
$$

One can express higher order correlation functions of the renewal process through its one-point correlation function and the renewal density. Indeed (see [DVJ], p. 136) for $t_{1} \leq t_{2} \leq \cdots \leq t_{k}$ and $k>1$ the following formula takes place

$$
\rho_{k}\left(t_{1}, \ldots, t_{k}\right)=\rho_{1}\left(t_{1}\right) \cdot u\left(t_{2}-t_{1}\right) \cdot u\left(t_{3}-t_{2}\right) \cdot \ldots \cdot u\left(t_{k}-t_{k-1}\right)
$$


It follows immediately from the above definitions that a random point field in $\mathbb{R}_{+}^{1}$ has i.i.d. nearest spacings iff it is a renewal process $(2.38)$. To make this process translation-invariant the probability density of $\tau_{0}$ must be given by

$$
\frac{1}{E \tau_{1}} \int_{x}^{+\infty} f(t) d t \quad([\mathrm{DVJ}], \text { p. } 72,[\mathrm{Fe}], \text { section XI.3) }
$$

Then one-point correlation function is identically constant, $\rho_{1}(x) \equiv \rho>0$, so (2.40) implies that the distribution of the process is uniquely defined by the renewal density (in particular one can obtain $\rho$ from $u(x)$ since $\rho=\left(E \tau_{1}\right)^{-1}$ and the Laplace transforms of $f$ and $u$ are simply related). Macchi ([Ma]) considered a special class of translation-invariant renewal processes with the interval distribution density

$$
f(x)=2 \rho(1-2 \rho \alpha)^{-\frac{1}{2}} e^{-\frac{x}{\alpha}} \cdot \sinh \left((1-2 \rho \alpha)^{\frac{1}{2}} \cdot\left(\frac{x}{\alpha}\right)\right),
$$

where

$$
2 \rho \alpha \leq 1, \rho>0, \alpha>0
$$

and showed that it is a determinantal random point field with the kernel

$$
K(x, y)=\rho \cdot \exp (-|x-y| / \alpha)
$$

(restrictions (2.43) are exactly $0<K \leq \mathrm{Id}$ ).

In the next theorem we classify all delayed renewal processes that are also determinantal random point fields in $\mathbb{R}_{+}^{1}$.

Theorem 6. Determinantal random point field in $\mathbb{R}_{+}^{1}$ with Hermitian kernel has i.i.d. spacings if and only if its kernel satisfies the following two conditions in addition to $0 \leq K \leq$ Id locally trace class :

a) for almost all $x_{1} \leq x_{2} \leq x_{3}$

$$
K\left(x_{1}, x_{2}\right) \cdot K\left(x_{2}, x_{3}\right)=K\left(x_{1}, x_{3}\right) \cdot K\left(x_{2}, x_{2}\right),
$$

b) for almost all $x_{1} \leq x_{2}$ the function

$$
K\left(x_{2}, x_{2}\right)-\frac{K\left(x_{1}, x_{2}\right) \cdot K\left(x_{2}, x_{1}\right)}{K\left(x_{2}, x_{1}\right)}
$$


depends only on the difference $x_{2}-x_{1}$. If a determinantal random point field is both translation-invariant and with i.i.d. spacings, it is given by (2.42)(2.44).

Remark 8. Of course a translation-invariant d.r.p.f. in $\mathbb{R}_{+}^{1}$ can be extended in a unique way to the translation-invariant d.r.p.f. in $\mathbb{R}^{1}$.

Proof of Theorem 6. First we prove the "only if" part of the theorem. Suppose that a determinantal random point field with a kernel $K(x, y)$ is also a delayed renewal process. From $(2.40), k=2,3$, we obtain the formula for the renewal density

$$
u(y-x)=K(y, y)-\frac{K(x, y) \cdot K(y, x)}{K(x, x)}, y \geq x,
$$

and the expression for $\rho_{3}\left(x_{1}, x_{2}, x_{3}\right), x_{1} \leq x_{2} \leq x_{3}$ :

$$
\begin{aligned}
& \rho_{3}\left(x_{1}, x_{2}, x_{3}\right)=K\left(x_{1}, x_{1}\right) \cdot u\left(x_{2}-x_{1}\right) \cdot u\left(x_{3}-x_{2}\right) \\
& \quad=K\left(x_{1}, x_{1}\right) \cdot\left(K\left(x_{2}, x_{2}\right)-\frac{K\left(x_{1}, x_{2}\right) \cdot K\left(x_{2}, x_{1}\right)}{K\left(x_{1}, x_{1}\right)}\right) \\
& \left(K\left(x_{3}, x_{3}\right)-\frac{K\left(x_{2}, x_{3}\right) \cdot K\left(x_{3}, x_{2}\right)}{K\left(x_{2}, x_{2}\right)}\right)
\end{aligned}
$$

Since with probability 1 there are no particles outside $A=\{x: K(x, x)>0\}$, we can always consider random point field restricted to $A$.

Comparing

$$
\rho_{3}\left(x_{1}, x_{2}, x_{3}\right)=\operatorname{det}\left(K\left(x_{i}, x_{j}\right)\right)_{1 \leq i, j \leq 3}
$$

with (2.48), we have

$$
\begin{aligned}
& K\left(x_{1}, x_{2}\right) \cdot K\left(x_{2}, x_{1}\right) \cdot K\left(x_{2}, x_{3}\right) \cdot K\left(x_{3}, x_{2}\right) \cdot \frac{1}{K\left(x_{2}, x_{2}\right)}= \\
& \quad-K\left(x_{1}, x_{3}\right) \cdot K\left(x_{3}, x_{1}\right) \cdot K\left(x_{2}, x_{2}\right)+K\left(x_{1}, x_{2}\right) \cdot K\left(x_{2}, x_{3}\right) \\
& \quad \cdot K\left(x_{3}, x_{1}\right)+K\left(x_{1}, x_{3}\right) \cdot K\left(x_{3}, x_{2}\right) \cdot K\left(x_{2}, x_{1}\right)
\end{aligned}
$$

which is equivalent to

$$
\begin{gathered}
\frac{1}{K\left(x_{2}, x_{2}\right)} \cdot\left(K\left(x_{1}, x_{2}\right) \cdot K\left(x_{2}, x_{3}\right)-K\left(x_{2}, x_{2}\right) \cdot K\left(x_{1}, x_{3}\right)\right) \\
\cdot\left(K\left(x_{3}, x_{2}\right) \cdot K\left(x_{2}, x_{1}\right)-K\left(x_{3}, x_{1}\right) \cdot K\left(x_{2}, x_{2}\right)\right)=0
\end{gathered}
$$


The third factor in the last equality is a complete conjugate of the second factor, and we obtain (2.45). Condition b) of the theorem has been already established in (2.47). For the translation-invariant d.r.p.f. the kernel $K(x, y)$ depends only on the difference, therefore $K(x, y)=\rho \cdot e^{-|x-y| / \alpha} \cdot e^{i \beta(x-y)}$, and the unitary equivalent kernel $e^{-i \beta x} K(x, y) \cdot e^{i \beta y}$ coincides with (2.44). Now we turn to the proof of the "if" part of the theorem. Once we are given the kernel satisfying (2.45) and (2.46) the candidate for the renewal density must obey $u\left(x_{2}-x_{1}\right)=K\left(x_{2}, x_{2}\right)-\frac{K\left(x_{1}, x_{2}\right) \cdot K\left(x_{2}, x_{1}\right)}{K\left(x_{1}, x_{1}\right)}$ for almost all $x_{1} \leq x_{2}$. Let $x_{1} \leq x_{2} \leq \cdots \leq x_{k}$. Our goal is to deduce the algebraic identity

$$
\begin{aligned}
& \operatorname{det}\left(K\left(x_{i}, x_{i}\right)\right)_{1 \leq i, j \leq k}=K\left(x_{1}, x_{1}\right) \cdot \prod_{i=1}^{k-1}\left(K\left(x_{i+1}, x_{i+1}\right)\right. \\
& \left.-\frac{K\left(x_{i}, x_{i+1}\right) \cdot K\left(x_{i+1}, x_{i}\right)}{K\left(x_{i}, x_{i}\right)}\right)
\end{aligned}
$$

from the basic identities between the commuting variables $K\left(x_{i}, x_{j}\right), \overline{K\left(x_{i}, x_{j}\right)}$ satisfying $K\left(x_{i}, x_{j}\right) \cdot K\left(x_{j}, x_{\ell}\right)=K\left(x_{i}, x_{\ell}\right) \cdot K\left(x_{j}, x_{j}\right), 1 \leq i \leq j \leq \ell \leq$ $k, K\left(x_{i}, x_{j}\right)=\overline{K\left(x_{j}, x_{i}\right)}$. Let us introduce $a(x)=K(x, x) \cdot K(0, x)^{-1}, b(x)=$ $K(0, x)^{-1}$. Then for $i \leq j$

$$
\begin{aligned}
& K\left(x_{i}, x_{j}\right)=a\left(x_{i}\right) \cdot b\left(x_{j}\right)^{-1}, \\
& K\left(x_{j}, x_{i}\right)=\overline{a\left(x_{i}\right)} \cdot{\overline{b\left(x_{j}\right)}}^{-1} .
\end{aligned}
$$

This allows us to write the determinant as

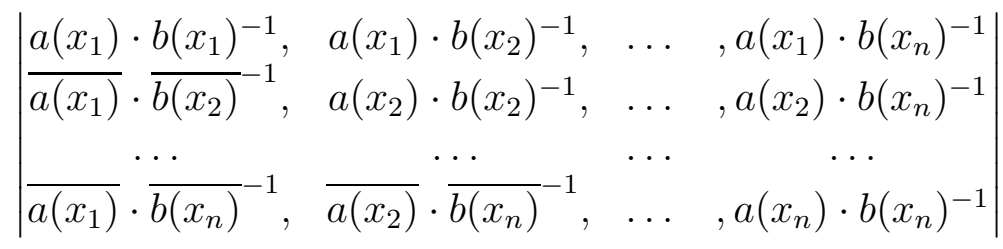

$$
\begin{aligned}
& =a\left(x_{1}\right) \cdot b\left(x_{1}\right)^{-1} \cdot \prod_{i=1}^{n-1}\left(b\left(x_{i+1}\right)^{-1} \cdot{\overline{b\left(x_{i+1}\right)}}^{-1}\right. \text {. } \\
& \left.\left(a\left(x_{i+1}\right) \cdot \overline{b\left(x_{i+1}\right)}-\overline{a\left(x_{i}\right)} \cdot b\left(x_{i}\right)\right)\right)
\end{aligned}
$$

which is exactly the r.h.s. of (2.50). Once we established $\rho_{k}\left(x_{1}, \ldots, x_{k}\right)=$ $\rho_{1}\left(x_{1}\right) \cdot \prod_{i=1}^{n-1} u\left(x_{i+1}-x_{i}\right), x_{1} \leq x_{2} \leq \cdots \leq x_{k}$, the rest of the proof is quite 
easy. Let $p_{k}\left(x_{1}, \ldots, x_{k}\right)$ be Janossy densities, i.e. the probability density of the event to have particles at $x_{1}, \ldots, x_{k}$, and no particles in between. We recall that $p_{k}\left(x_{1}, \ldots, x_{k}\right)=\sum_{j=1}^{\infty} \frac{(-1)^{j}}{j !} \cdot \int \rho_{k+j}\left(x_{1}, \ldots, x_{k} ; y_{k+1}, \ldots, y_{k+j}\right) d y_{k+1} \ldots d y_{k+j}$, where the integration in the $j$ th term is over $\left(x_{1}, x_{k}\right) \times \cdots \times\left(x_{1}, x_{k}\right)$. We claim that

$$
p_{k}\left(x_{1}, \ldots, x_{k}\right)=\rho_{1}\left(x_{1}\right) \cdot \prod_{i=1}^{k-1} f\left(x_{i+1}-x_{i}\right)
$$

where the interval distribution density $f$ and the renewal distribution density $u$ are related via the convolution equation

$$
u=f+u * f
$$

Theorem 6 is proven.

Remark 9. The analogue of Theorem 6 is valid in the discrete case and the proof is the same. One has to replace (2.42) by the solution of the discrete convolution equation (2.53) with $u(n)=1-\rho \cdot e^{-2 \beta n}, K\left(n_{1}, n_{2}\right)=$ $\rho \cdot e^{-\beta\left|n_{1}-n_{2}\right|}, 0<\rho \leq 1, \beta>0$, so that

$$
\hat{f}(t)=\sum_{n=0}^{\infty} f(n) e^{i n t}=\frac{(1-\rho)-\left(e^{-2 \beta}-\rho\right) \cdot e^{i t}}{(2-\rho)-\left(2 e^{-2 \beta}-\rho+1\right) \cdot e^{i t}+e^{-2 \beta} e^{2 i t}}
$$

Remark 10. One can consider a generalization of Theorem 6 to the case when the multiplicative identity (2.45) still holds, but the renewal density

$$
u\left(x_{1}, x_{2}\right)=K\left(x_{2}, x_{2}\right)-\frac{K\left(x_{1}, x_{2}\right) \cdot K\left(x_{2}, x_{1}\right)}{K\left(x_{1}, x_{1}\right)}
$$

no longer depends only on the difference of $x_{1}$ and $x_{2}$. Such processes have independent but not necessarily identically distributed spacings since the spacings distribution $f(x, y) d y$ depends on the position $x$ of the left particle. Thus

$$
\begin{aligned}
& u\left(x_{1}, x_{2}\right)=f\left(x_{1}, x_{2}\right)+\int_{x_{1}}^{x_{2}} f\left(x_{1}, y_{1}\right) \cdot f\left(y_{1}, x_{2}\right) d y_{1} \\
& \quad+\int_{x_{1}}^{x_{2}} \int_{x_{1}}^{y_{2}} f\left(x_{1}, y_{1}\right) \cdot f\left(y_{1}, y_{2}\right) \cdot f\left(y_{2}, x_{2}\right) d y_{1} d y_{2}+\ldots
\end{aligned}
$$


where $f(x, y)$ is a one-parameter family of probability densities, such that

$$
\operatorname{supp} f(x, \cdot) \subset[x,+\infty], f \geq 0, \int f(x, y) d y=1 .
$$

We recall the inversion formula for the equation (2.55):

$$
\begin{aligned}
& f\left(x_{1}, x_{2}\right)=u\left(x_{1}, x_{2}\right)-\int_{x_{1}}^{x_{2}} u\left(x_{1}, y_{1}\right) u\left(y_{1}, x_{2}\right) d y_{1} \\
& \quad+\int_{x_{1}}^{x_{2}} \int_{x_{1}}^{y_{2}} u\left(x_{1}, y_{1}\right) \cdot u\left(y_{1}, y_{2}\right) \cdot u\left(y_{2}, x_{2}\right) d y_{1} d y_{2}-\ldots
\end{aligned}
$$

Writing $K(x, y)=a(x) b(y)^{-1}, x \leq y$, where $a(x)=\frac{K(x, x)}{K(0, x)}, b(y)=\frac{1}{K(0, y)}$, and $u(x, y)=\frac{1}{|b(y)|^{2}} \cdot(a(y) \overline{b(y)}-\overline{a(x)} b(x))$, one can in principle characterize through (2.56) the class of corresponding interval densities $u(x, y)$.

\subsection{Plancherel Measure on Partitions and its Generali- zations-z-Measures and Schur Measures}

By a partition of $n=1,2, \ldots$ we understand a collection of non-negative integers $\lambda=\left(\lambda_{1}, \ldots, \lambda_{m}\right)$ such that $\lambda_{1}+\cdots+\lambda_{m}=n$ and $\lambda_{1} \geq \lambda_{2} \geq \cdots \geq \lambda_{m}$. We shall use a notation $\operatorname{Par}(n)$ for the set of all partitions of $n$. For the basic facts about partitions we refer the reader to [St], [Fu], [Mac], [Sa]. In particular recall that each partition $\lambda$ of $n$ (denoted $\lambda \vdash n$ ) can be identified with a Young diagram with $|\lambda|=n$ boxes. A partition $\lambda^{\prime}$ corresponds to the transposed diagram. Let $d$ be the number of diagonal boxes in $\lambda$ (i.e., the number of diagonal boxes in the Young diagram corresponding to $\lambda$ ). We define the Frobenius coordinates of $\lambda$ as $\left(p_{1}, \ldots, p_{d} \mid q_{1}, \ldots, q_{k}\right)$, where $p_{j}=\lambda_{j}-j, q_{j}=\lambda_{j}^{\prime}-j, j=1, \cdots, d$. The importance of partitions in Representation Theory can be most easily understood from the fact that the elements of $\operatorname{Par}(n)$ label the irreducible representations of the symmetric group $S_{n}$ (see e.g., [Sa], [Fu]). The Plancherel measure $M_{n}$ on the set $\operatorname{Par}(n)$ of all partitions of $n$ is given by

$$
M_{n}(\lambda)=\frac{(\operatorname{dim} \lambda)^{2}}{n !}
$$

where $\operatorname{dim} \lambda$ is the dimension of the corresponding representation of $S_{n}$. The dimension $\operatorname{dim} \lambda$ can be expressed in terms of the Frobenius coordinates via 
a determinantal formula

$$
\frac{\operatorname{dim} \lambda}{n !}=\operatorname{det}\left[\frac{1}{\left(p_{i}+q_{j}+1\right) \cdot p_{i} ! \cdot q_{i} !}\right]_{1 \leq i, j \leq d}
$$

where $|\lambda|=n\left([\mathrm{Ol}]\right.$, Proposition 2.6, formula (2.7)). Let $\operatorname{Par}=\bigsqcup_{n=0}^{\infty} \operatorname{Par}(n)$. Consider the measure $M^{\theta}$ on Par, which in analogy with statistical mechanics can be called the grand canonical ensemble:

$$
\begin{aligned}
& M^{\theta}(\lambda)=e^{-\theta} \cdot \frac{\theta^{n}}{n !} M_{n}(\lambda), \\
& \text { if } \lambda \in \operatorname{Par}(n), n=0,1,2, \ldots, 0 \leq \theta<\infty .
\end{aligned}
$$

$M^{\theta}$ is also called the poissonization of the measures $M_{n}$. It follows from (2.59) that $|\lambda|$ is distributed by the Poisson law with the mean $\theta$, and $M^{\theta}(|| \lambda \mid=$ $n)=M_{n}$. In the Frobenius coordinates measures $M^{\theta}, M_{n}$ can be viewed as random point fields on the lattice $\mathbb{Z}^{1}$. Recently Borodin, Okounkov and Olshanski ([BOO]) and, independently, Johansson [Jo3]) proved that $M^{\theta}$ is a determinantal random point field (to be exact in [Jo3] only the restriction of $M^{\theta}$ to the first half of the Frobenius coordinates $\left(p_{1}, \ldots, p_{d(\lambda)}\right)$ was studied, and as a result, only the part of (2.60) corresponding to $x y>0$ was obtained). To formulate the results of [BOO], [Jo3] we define the modified Frobenius coordinates of $\lambda$ by

$$
\operatorname{Fr}(\lambda):=\left\{p_{1}+\frac{1}{2}, \ldots, p_{d}+\frac{1}{2},-q_{1}-\frac{1}{2}, \ldots,-q_{d}-\frac{1}{2}\right\} .
$$

Let $\rho_{k}^{\theta}\left(x_{1}, \ldots, x_{k}\right)$ be the $k$-point correlation function of $M^{\theta}$ in the modified Frobenius coordinates, where

$$
\left\{x_{1}, \ldots, x_{k}\right\} \subset \mathbb{Z}^{1}+\frac{1}{2}
$$

Then

$$
\rho_{k}^{\theta}\left(x_{1}, \ldots, x_{k}\right)=\operatorname{det}\left[K\left(x_{i}, x_{j}\right)\right]_{1 \leq i, j \leq k},
$$

where $K$ is a so-called discrete Bessel kernel,

$$
K(x, y)= \begin{cases}\sqrt{\theta} \cdot \frac{J_{|x|-\frac{1}{2}}(2 \sqrt{\theta}) \cdot J_{|y|+\frac{1}{2}}(2 \sqrt{\theta})-J_{|x|+\frac{1}{2}}(2 \sqrt{\theta}) \cdot J_{|y|-\frac{1}{2}}(2 \sqrt{\theta})}{|x|-|y|}, \text { if } x \cdot y>0, \\ \sqrt{\theta} \cdot \frac{J_{|x|-\frac{1}{2}}(2 \sqrt{\theta}) \cdot J_{|y|-\frac{1}{2}}(2 \sqrt{\theta})-J_{|x|+\frac{1}{2}}(2 \sqrt{\theta}) \cdot J_{|y|+\frac{1}{2}}(2 \sqrt{\theta})}{x-y}, \text { if } x \cdot y<0\end{cases}
$$


where $J_{x}(\cdot)$ is the Bessel function of order $x$. We note that the kernel $K(x, y)$ is not Hermitian symmetric, however the restriction of this kernel to the positive and negative semi-axis is Hermitian. (2.60) can be seen as a limiting case of a more general theorem obtained by Borodin and Olshanski for the so-called $z$-measures (see Theorem 3.3 of [BO1], also [BO2], [BO3], [KOV] and references therein). Let $z, z^{\prime}$ be complex numbers such that either

$$
\begin{aligned}
& z^{\prime}=\bar{z} \in \mathbb{C} \backslash \mathbb{Z} \\
& \text { or } \\
& {[z]<\min \left(z, z^{\prime}\right) \leq \max \left(z, z^{\prime}\right)<[z]+1}
\end{aligned}
$$

where $z, z^{\prime}$ real and [ ] denotes the integer part. Let $(x)_{j}=x \cdot(x+1) \cdot \ldots$. $(x+j-1),(x)_{0}=1$. Below we introduce a 2 -parametric family of probability measures $M_{z, z^{\prime}}^{(n)}$ on $\operatorname{Par}(n)$. These measures take their origin in harmonic analysis on the infinite symmetric group ([KOV], $[\mathrm{Ol}])$. By definition

$$
\begin{aligned}
& M_{z, z^{\prime}}^{(n)}(\lambda)=\frac{\left(z \cdot z^{\prime}\right)^{d(\lambda)}}{\left(z \cdot z^{\prime}\right)_{n}} \cdot \prod_{i=1}^{d(\lambda)}(z+1)_{p_{i}} \cdot\left(z^{\prime}+1\right)_{p_{i}} . \\
& (-z+1)_{q_{i}} \cdot\left(-z^{\prime}+1\right)_{q_{i}} \cdot \frac{\operatorname{dim}^{2} \lambda}{|\lambda| !}
\end{aligned}
$$

The conditions on $z, z^{\prime}$ stated above are equivalent to the requirement that $(z)_{j} \cdot\left(z^{\prime}\right)_{j}$ and $(-z)_{j} \cdot\left(-z^{\prime}\right)_{j}$ are positive for any $j=1,2, \ldots$ We note that $M_{z, z^{\prime}}^{(n)}$ converges to the Plancherel measure $M_{n}$ if $z, z^{\prime} \rightarrow \infty$. The measure $M_{z, z^{\prime}}^{(n)}$ is called the $n$-th level $z$-measure. Consider now the negative binomial distribution on the non-negative integers

$$
(1-\xi)^{z \cdot z^{\prime}} \cdot \frac{\left(z \cdot z^{\prime}\right)_{n}}{n !} \xi^{n}, n=0,1, \ldots
$$

where $\xi$ is an additional parameter, $0<\xi<1$. The corresponding mixture of the $n$-level $z$-measures defines measure $M_{z, z^{\prime}, \xi}$ on Par. We remark that $M_{z, z^{\prime}, \xi}$ degenerates into $M^{\theta}$ if $z, z^{\prime} \rightarrow \infty, \xi \rightarrow 0$ in such a way that $z z^{\prime} \xi \rightarrow \theta$. It was shown in [BO1] that in the modified Frobenius coordinates $M_{z, z^{\prime}, \xi}$ is a determinantal random point field on $\mathbb{Z}^{1}+\frac{1}{2}$. The corresponding kernel can be expressed in terms of the Gauss hypergeometric function and is called the hypergeometric kernel. It appears that a number of familiar kernels can be obtained in terms of the hypergeometric kernel, in particular Hermite 
kernel ((2.2), (2.3), (2.7)), Laguerre kernel ((2.2), (2.28)), Meixner kernel ((2.67) below), Charlier kernel. For the hierarchy of the degenerations of the hypergeometric kernel we refer the reader to [BO2] §9. Recently Okounkov [Ok1] showed that the measures $M_{z, z^{\prime}, \xi}$ are the special case of an infinite parameter family of probability measures on Par, called the Schur measures, and defined as

$$
M(\lambda)=\frac{1}{z} s_{\lambda}(x) \cdot s_{\lambda}(y),
$$

where $s_{\lambda}$ are the Schur functions (for the definition of the Schur functions see [St] or $[\mathrm{Mac}]), x=\left(x_{1}, x_{2}, \ldots\right)$ and $y=\left(y_{1}, y_{2}, \ldots\right)$ are parameters such that

$$
Z=\sum_{\lambda \in \operatorname{Par}} s_{\lambda}(x) \cdot s_{\lambda}(y)=\prod_{i, j}\left(1-x_{i} y_{j}\right)^{-1}
$$

is finite and $\left\{x_{i}\right\}_{i=1}^{\infty}=\overline{\left\{y_{i}\right\}_{i=1}^{\infty}}$. Measures $M_{z, z^{\prime}, \xi}$ formally correspond to $\sum_{i=1}^{\infty} x_{i}^{m}=\xi^{\frac{m}{2}} \cdot z, \sum_{i=1}^{\infty} y_{i}^{m}=\xi^{\frac{m}{2}} \cdot z^{\prime}, m=1,2 \ldots$.To be precise one should consider the Newton power sums as real parameters and express the Schur functions as polynomials in the power sums. By now the reader probably would not be very surprised to learn that the Schur measures also can be considered as determinantal random point fields ([Ok1], Theorems 1,2)!

\subsection{Two-Dimensional Random Growth Model}

As our last example we consider the following two-dimensional random growth model ([Jo2]). Let $\left\{a_{i j}\right\}_{i, j \geq 1}$ be a family of independent identically distributed random variables with a geometric law

$$
p\left(a_{i j}=k\right)=p \cdot q^{k}, k=0,1,2, \ldots
$$

where $0<q<1, p=1-q$. One may think about (2.65) as the distribution of the first success time in a series of Bernoulli trials. We define

$$
G(M, N)=\max _{\pi} \sum_{(i, j) \in \pi} a_{i j},
$$

where the maximum in (2.66) is considered over all up/right paths $\pi$ from $(1,1)$ to $(M, N)$, in other words over $\pi=\left\{\left(i_{1}, j_{1}\right)=(1,1),\left(i_{2}, j_{2}\right),\left(i_{3}, j_{3}\right), \ldots\right.$, 
$\left.\left(i_{M+N-1}, j_{M+N-1}\right)=(M, N)\right\}$, such that $\left(i_{k+1}, j_{k+1}\right)-\left(i_{k}, j_{k}\right) \in\{(0,1),(1,0)\}$. We mention in passing that distribution of random variables $\{G(M, N)\}$ can be interpreted in terms of randomly growing Young diagrams and totally asymmetric exclusion process with discrete time (for the details see [Jo2]). Without loss of generality we may assume $M \geq N \geq 1$. To state explicitly the connection to the determinantal random point fields we introduce the discrete weight $w_{K}^{q}(x)=\left(\begin{array}{c}x+K-1 \\ x\end{array}\right) \cdot q^{x}, K=M-N+1$, on non-negative integers $x=0,1,2 \ldots$ The normalized orthogonal polynomials $\left\{M_{n}(x)\right\}_{n \geq 0}$ with respect to the weight $w_{K}^{q}$ are proportional to the classical Meixner polynomials $([\mathrm{Ch}])$. The kernel

$$
K_{M, N}(x, y)=\sum_{j=0}^{N-1} M_{j}(x) M_{j}(y)\left(w_{K}^{q}(x) w_{K}^{q}(y)\right)^{\frac{1}{2}}
$$

satisfies the conditions of Lemma 4 with respect to the counting measure on non-negative integers. Therefore

$$
P_{N}\left(x_{1}, \ldots, x_{N}\right)=\frac{1}{N !} \operatorname{det}\left(K_{M, N}\left(x_{i}, x_{j}\right)\right)_{1 \leq i, j \leq N}
$$

defines a discrete determinantal random point field. It was shown by Johansson that the distribution of the random variable $G(M, N)$ coincides with the distribution of the right-most particle in (2.68). After appropriate rescaling in the limit $N \rightarrow \infty, M \rightarrow \infty, \frac{M}{N} \rightarrow$ const, this distribution converges to the distribution of the right-most particle in the Airy random point field (2.36). Additional information on the subject of the last two subsections can be found in the recent papers/preprints [AD], [BDJ1], [BDJ2], [BR1], [BR2], [BR3], [Bor], [ITW], [Ku], [Ok2], [PS1], [PS2], [TW5], [TW6].

\section{Translation Invariant Determinantal Ran- dom Point Fields}

As before $(X, \mathcal{B}, P)$ denotes a random point field with a one-particle space $E$, hence $X$ is a space of locally finite configurations of particles in $E, \mathcal{B}$ is a Borel $\sigma$-algebra of measurarble subsets of $X$ and $P$ is a probability measure on $(X, B)$. Throughout this section we always assume $E=\mathbb{R}^{d}$ or $\mathbb{Z}^{d}$. We define a continuous action $\left\{T^{t}\right\}_{t \in E}$ of $E$ on $X$ in a natural way:

$$
T^{t}: X \rightarrow X,\left(T^{t} \xi\right)_{i}=(\xi)_{i}+t .
$$


Definition 5. Random point field $(X, \mathcal{B}, P)$ is called translation invariant if for any $A \in \mathcal{B}$, any $t \in E$

$$
P\left(T^{-t} A\right)=P(A)
$$

The translation invariance of a random point field implies the invariance of $k$-point correlation functions:

$$
\begin{aligned}
& \rho_{k}\left(x_{1}+t, \ldots, x_{k}+t\right)=\rho_{k}\left(x_{1}, \ldots, x_{k}\right), \text { a.e. } \\
& k=1,2, \ldots, t \in E .
\end{aligned}
$$

Conversely, if $\left\{\rho_{k}\right\}$ are invariant under $\left\{T^{t}\right\}$, then there exists a corresponding random point field which is translation invariant ([L3]). In particular, if the translation invariant correlation functions define $P$ uniquely then the random point field is translation invariant. In the case of a determinantal random point field this implies the following criterion: a determinantal random point field is translation invariant if and only if the kernel $K$ is translation invariant, i.e., $K(x, y)=K(x-y, 0)=: K(x-y)$. In this section we restrict our attention to the translation invariant determinantal random point fields. We are interested in the ergodic properties of the dynamical system $\left(X, B, P,\left\{T^{t}\right\}\right)$. For the convenience of the reader recall some basic definitions of Ergodic Theory ([CFS]).

- A dynamical system is said to be ergodic if the measure $P(A)$ of any invariant set $A$ equals 0 or 1 .

- A dynamical system has the mixing property of multiplicity $r \geq 1$ if for any functions $f_{0}, f_{1}, \ldots, f_{r} \in L^{r+1}(X, \mathcal{B}, P)$ we have

$$
\lim _{t_{1}, \ldots, t_{r} \rightarrow \infty} \int_{X} f_{0}(\xi) f_{1}\left(T^{t_{1}} \xi\right): \cdots: f_{r}\left(T^{t_{1}+\cdots+t_{r}} \xi\right) d F=\prod_{i=0}^{r} \int_{X} f_{i}(\xi) d P
$$

- A dynamical system has an absolute continuous spectra if for any $f \in$ $L^{2}(X, B, P)$ orthogonal to constants

$$
\int_{X} f(\xi) \overline{f\left(T^{t} \xi\right)} d P=\int e^{i(t \cdot \lambda)} h_{f}(\lambda) d \lambda ;
$$


where the integration at the r.h.s. of (3.4) is over $\mathbb{R}^{d}$ in the continuous case and over $[0,2 \pi]^{d}$ in the discrete case, and $h_{f}(\lambda) d \lambda$ is a finite measure absolutely continuous with respect to the Lebesgue measure. One can interpret (3.4) in the following way. We define a $d$-parameter group of unitary operators $\left\{U^{t}\right\}_{t \in E}$ on $L^{2}(X, B, P)$ as

$$
\left(U^{t} f\right)(\xi)=f\left(T^{t} \xi\right)
$$

Usually such family of unitary operators is called adjoint to the dynamical system. It is easy to see that $\left\{U^{t}\right\}$ commute. Since $L^{2}(X, \mathcal{B}, P)$ is separable and $\left(U^{t} \psi, \varphi\right)$ is a measurable function of $t$ for any $\psi, \varphi \in$ $L^{2}(X, B, P)$ one can apply the von Neumann theorem ([RS], vol. 1, Theorem VIII.9) to conclude that $U^{t}$ is strongly continuous. In the case $E=\mathbb{R}^{d}$ one has $h_{f}(\lambda) d \lambda=d\left(f, Q_{\lambda} f\right)$, where $d Q_{\lambda}$ is a projection-valued measure, $Q_{\lambda}=Q_{\left(-\infty, \lambda_{1}\right) \times \cdots \times\left(-\infty, \lambda_{d}\right)}=\prod_{j=1}^{d} \chi_{\left(-\infty, \lambda_{j}\right)}\left(A_{j}\right),\left\{A_{j}\right\}_{j=1}^{d}$ are the generators of the one-parameter groups $U^{\left(0, \ldots, t_{j}, 0, \ldots 0\right)}$ and $\chi_{(-\infty, t)}$ is the indicator of $(-\infty, t)$ ([RS], vol. I, Theorem VIII.12). In the discrete case $E=\mathbb{Z}^{d} \quad d Q_{\lambda}$ is a projection-valued measure on a $d$-dimensional torus,

$$
Q_{\left[1, e^{i \lambda_{1}}\right] \times \cdots \times\left[1, e^{i \lambda d}\right]}=\prod_{j=1}^{d} \chi_{\left[1, e^{i \lambda_{j}}\right]}\left(U_{j}\right), U_{j}=U^{\left(0, \ldots, t_{j}=1, \ldots 0\right)} .
$$

Theorem 7. Let $(X, B, P)$ be a translation invariant determinantal random point field. Then the dynamical system $\left(X, B, P,\left\{T^{t}\right\}\right)$ is ergodic, has the mixing property of any multiplicity and its spectra is absolutely continuous.

Remark 11. Recall that the absolute continuity of the spectra implies the mixing property of multiplicity 1, which in turn implies ergodicity ([CFS]).

Proof of Theorem 7. We note that the linear combinations of

$$
\begin{aligned}
& f(\xi)=\prod_{j=1}^{N} S_{g_{j}}(\xi), \\
& N \geq 1, S_{g}(\xi)=\sum_{i} g\left(x_{i}\right), g_{j} \in C_{0}^{\infty}\left(\mathbb{R}^{d}\right), j=1, \ldots N
\end{aligned}
$$

are dense in $L^{2}(X, B, P)$. Therefore it is enough to establish (3.3), (3.4) for the functions of such form. We start with the lemma calculating the mathematical expectation of (3.5). 


\section{Lemma 5.}

a)

$$
\begin{aligned}
& \mathbb{E}_{P} \prod_{j=1}^{N} S_{g_{j}}(\xi)=\sum_{m=1}^{N} \sum_{\substack{\text { over partitions } \\
\bigsqcup_{\ell=1}^{m} C_{\ell}=\{1, \ldots, N\}}} \prod_{\ell=1}^{m}\left[\sum _ { k _ { \ell } = 1 } ^ { \# ( C _ { \ell } ) } \sum _ { \substack { \text { over partitions } \\
\bigsqcup _ { i = 1 } ^ { k _ { \ell } } B _ { \ell i } = C _ { \ell } } } \left\{\sum_{\sigma \in S^{k_{\ell}}}\right.\right. \\
& \left.\left.\frac{(-1)^{\sigma}}{k_{\ell}} \cdot \int \prod_{i=1}^{k_{\ell}} g_{B_{\ell \sigma(i)}}\left(x_{i}\right) \cdot K\left(x_{i+1}-x_{i}\right) d x_{1} \ldots d x_{k_{\ell}}\right\}\right]
\end{aligned}
$$

where $g_{B_{\ell i}}(x)=\prod_{j \in B_{\ell i}} g_{j}(x)$.

b) $\mathbb{E} \prod_{j=1}^{N_{1}+\cdots+N_{r+1}} S_{g_{j}}(\xi)-\prod_{s=1}^{r+1}\left(\mathbb{E} \prod_{N_{1}+\cdots+N_{s-1}+1}^{N_{1}+\cdots+N_{s}} S_{g_{j}}(\xi)\right)=$ (similar expression to (3.6), with the only difference that partitions

$$
\bigsqcup_{\ell=1}^{m} C_{\ell}=\left\{1,2, \ldots, \sum_{s=1}^{r+1} N_{s}\right\}
$$

satisfy $\left.\left({ }^{*}\right)\right)$, where

(*) There exists at least one element $C_{\ell}$ of the partition such that the intersections of $C_{\ell}$ with at least two of the following sets $\left\{1, \ldots N_{1}\right\}, \ldots,\left\{N_{1}+\right.$ $\left.\cdots+N_{s-1}+1, \ldots, N_{1}+\cdots+N_{s}\right\}, \ldots,\left\{N_{1}+\cdots+N_{r}+1, \ldots, N_{1}+\cdots+N_{r+1}\right\}$ are non-empty.

Proof of Lemma 5. The proof of part a) is rather straightforward and quite similar to the one given at the beginning of $\S 2$ in [So3] (see formulas (2.1)-(2.7) from the reference). The proof of part b) follows from a).

To derive the mixing property (3.3) we replace $g_{j}(\cdot)$ for $N_{1}+\cdots+N_{s-1}+$ $1 \leq j \leq N_{1}+\cdots+N_{s}, s=1, \ldots, r+1$, in (3.7) by $g_{j}\left(\cdot+t_{1}+\cdots+t_{s-1}\right)$. Fix a partition $\bigsqcup_{\ell=1}^{m} C_{\ell}=\left\{1,2, \ldots, N_{1}+\cdots+N_{r+1}\right\}$. Since $\left\{g_{j}\right\}$ are bounded functions with compact support, each of $m$ factors at the r.h.s. of (3.7) is bounded. We claim that the $\ell^{\text {th }}$ factor (corresponding to $C_{\ell}$, where $\ell$ is the same index as in $(*))$ goes to zero. To see this we fix an arbitrary partition of $C_{\ell}, \bigsqcup_{i=1}^{k_{\ell}} B_{\ell i}=C_{\ell}$. By assumption, $C_{\ell}$ contains indices $1 \leq$ $u<v \leq N_{1}+\cdots+N_{r+1}$, such that $u$ and $v$ belong to different subsets $\left\{1, \ldots, N_{1}\right\}, \ldots\left\{N_{1}+\cdots+N_{s-1}+1, \ldots, N_{1}+\cdots+N_{s}\right\}, \ldots,\left\{N_{1}+\cdots+N_{r}+\right.$ $\left.1, \ldots, N_{1}+\cdots+N_{r+1}\right\}$. We claim that

$$
\int \prod_{i=1}^{k_{\ell}} g_{B_{\ell \sigma(i)}}\left(x_{i}\right) \cdot K\left(x_{i+1}-x_{i}\right) d x_{1} \ldots d x_{k_{\ell}}
$$


goes to zero as $\min \left\{t_{s}, 1 \leq s \leq r\right\} \rightarrow \infty$. Indeed if $\min \left\{t_{s}, 1 \leq s \leq r\right\}$ is sufficiently large, the indices $u, v$ belong to different $B_{\ell i}$ 's or the corresponding $g_{B_{\ell i}}$ is zero (the supports of the factors in $g_{B_{\ell i}}$ will not intersect). Once $u$ and $v$ belong to different $B_{\ell i}$ 's the argument in $K\left(x_{i+1}-x_{i}\right)$ for some $i$ is greater than $\min \left\{t_{s}, 1 \leq s \leq r\right\}$. Since the Fourier transform of $K(x), \hat{K}(t)=$ $\int e^{i x t} K(x) d x$ is a non-negative integrable function (bounded from above by 1 ), applying the Riemann-Lebesgue lemma we obtain that $K\left(x_{i+1}-x_{i}\right)$ goes to zero. The other terms in (3.8) are bounded and the integration is over a bounded set, therefore (3.8) goes to zero and the proof of the mixing property follows.

To establish the absolute continuity of the spectrum we apply (3.7) when $r=2, N_{1}=N_{2}=N, g_{N+j}(x)=\overline{g_{j}(x+t)}, j=1, \ldots, N, f(\xi)=\prod_{j=1}^{N} S_{g_{j}}(\xi)$, $\overline{f\left(T^{t} \xi\right)}=\prod_{j=1}^{N} S_{\overline{g_{j}}}\left(T^{t} \xi\right)=\prod_{j=N+1}^{2 N} S_{\overline{g_{j}}}(\xi)$. We have

$$
\begin{aligned}
& \mathbb{E}(f(\xi)-\mathbb{E} f) \cdot\left(\overline{f\left(T^{t} \xi\right)}-\overline{\mathbb{E} f}\right)=\sum_{\substack{m=1 \\
\bigsqcup_{\ell=1}^{m} C_{\ell}=\{1, \ldots, 2 N\}}}^{2 N} \sum_{\substack{\text { over partitions } \\
C_{\ell}}}^{*}\left[\sum_{k_{\ell}=1}^{\#\left(C_{\ell}\right)}\right. \\
& \left.\sum_{\substack{\text { over partitions } \\
\bigsqcup_{i=1}^{k_{\ell}} B_{\ell i}=C_{\ell}}}\left\{\sum_{\sigma \in S^{k} \ell} \frac{(-1)^{\sigma}}{k_{\ell}} \cdot \int \prod_{i=1}^{k_{\ell}} g_{B_{\ell \sigma(i)}}\left(x_{i}\right) \cdot K\left(x_{i+1}-x_{i}\right) d x_{1} \ldots d x_{k_{\ell}}\right\}\right],
\end{aligned}
$$

where we assume that $x_{k_{\ell}+1}=x_{1}$ in the integral, and the sume in $\sum^{*}$ is over partitions $\left\{C_{1}, \ldots, C_{m}\right\}$ such that for at least one element $C_{\ell}$ of the partition both $C_{\ell} \cap\{1,2, \ldots, N\}$ and $C_{\ell} \cap\{N+1, \ldots, 2 N\}$ are non-empty (we denoted above this property by $(*))$. The terms in the product $\prod_{\ell=1}^{m}$ corresponding to those $\ell$ that do not satisfy $\left(^{*}\right)$ are constants as functions of $t$. Fix now $\ell$ satisfying $(*)$. We claim that

$$
\begin{aligned}
& \int \prod_{i=1}^{k_{\ell}} g_{B_{\ell \sigma(i)}}\left(x_{i}\right) \cdot K\left(x_{i+1}-x_{i}\right) d x_{1} \ldots d x_{k_{\ell}}= \\
& \left(\frac{1}{2 \pi}\right)^{k_{\ell}} \prod_{i=1}^{k_{\ell}} \widehat{g}_{B_{\ell \sigma(i)}}\left(y_{i+1}-y_{i}\right) \cdot \hat{K}\left(y_{i+1}\right) d y_{1} \ldots d y_{k_{\ell}}
\end{aligned}
$$

can be written as $\int e^{i(t \cdot \lambda)} h(\lambda) d \lambda$, where $h(\lambda)$ is an integrable function. The check is rather straightforward and we leave the details to the reader. We infer that (3.9) is a linear combination of the products of the Fourier transforms of integrable functions. Since the product of the Fourier transforms is 
the Fourier transform of the convolution the proof of the absolute continuity of the spectrum follows. Theorem 7 is proven.

One can without difficulty calculate the spectral density of the centralized linear statistics

$$
S_{g}(\xi)-\mathbb{E} S_{g}=\sum_{i} g\left(x_{i}\right)-\mathbb{E} \sum_{i} g\left(x_{i}\right)
$$

Namely

$$
\begin{aligned}
& \mathbb{E}\left(S_{g}-\mathbb{E} S_{g}\right)\left(\overline{S_{g}\left(T^{t} \cdot\right)}-\mathbb{E} \overline{S_{g}}\right)=\int e^{i(t \lambda)} \cdot\left(K(0)-\widehat{|K|^{2}}(\lambda)\right) \\
& \cdot \frac{1}{2 \pi}|\hat{g}(\lambda)|^{2} d \lambda, \text { and } h_{S_{g}}(\lambda)=\left(K(0)-\widehat{\left.K\right|^{2}}(\lambda)\right) \cdot \frac{1}{2 \pi}|\hat{g}(\lambda)|^{2}
\end{aligned}
$$

We conclude that

$$
\mu(d \lambda)=\left(K(0)-\widehat{|K|^{2}}(\lambda)\right) d \lambda
$$

is the spectral measure of the restriction of $\left\{U^{t}\right\}$ to the subspace of the centralized linear statistics. Since $0 \leq \hat{K}(\lambda) \leq 1, K(0)=\frac{1}{2 \pi} \int \hat{K}(\lambda) d \lambda$, we see that

$$
0 \leq \frac{d \mu}{d \lambda}=K(0)-\widehat{|K|^{2}}(\lambda)=K(0)-\frac{1}{2 \pi} \int \hat{K}(y) \hat{K}(y-\lambda) d y \leq K(0)
$$

We note that $\frac{d \mu}{d \lambda}>0$ for $\lambda \neq 0$, and $\frac{d \mu}{d \lambda}(0)=0$ if and only if $\hat{K}(\lambda)$ is an indicator. In particular the spectral measure $\mu$ is equivalent to the Lebesgue measure.

Before we formulate the next lemma recall that by $\#_{[-L, L]^{d}}(\xi)$ we denote the number of particles in $[-L, L]^{d}$.

\section{Lemma 6.}

$$
\operatorname{Var}\left(\#_{[-L, L]^{d}}\right)=\operatorname{Vol}\left([-L, L]^{d}\right) \cdot\left(\frac{d \mu}{d \lambda}(0)+\bar{o}(1)\right) \text { as } L \rightarrow \infty .
$$

Proof of Lemma 6. The probabilitists are well familiar with the analogue of this result in the Theory of Random Processes: let $\left\{\eta_{n}\right\}$ be $L^{2}$ stationary random sequence and $h(\lambda)$ its spectral density, $\mathbb{E} \eta_{n} \overline{\eta_{m}}=b(n-$ 
$m)=\frac{1}{2 \pi} \int_{0}^{2 \pi} e^{i \lambda \cdot(n-m)} h(\lambda) d \lambda$, then $\operatorname{Var}\left(\eta_{n}+\cdots+\eta_{n}\right)=(h(0)+\bar{o}(1)) \cdot n([\mathrm{IL}]$, section XVIII.2). To prove the lemma we write

$$
\begin{aligned}
& \operatorname{Var}\left(\#_{[-L, L]^{d}}\right)=\int_{[-L, L]^{d}} \int_{[-L, L]^{d}} \rho_{2}(x, y)-\rho_{1}(x) \rho_{1}(y) d x d y \\
& +\int_{[-L, L]^{d}} \rho_{1}(x) d x=-\int_{[-L, L]^{d}} \int_{[-L, L]^{d}}|K|^{2}(x-y) d x d y \\
& +K(0) \operatorname{Vol}\left([-L, L]^{d}\right)=\left(K(0)-\int_{\mathbb{R}^{d}}|K|^{2}(x) d x+\bar{o}(1)\right) \cdot \operatorname{Vol}\left([-L, L]^{d}\right)= \\
& \left(K(0)-\widehat{|K|^{2}}(0)+\bar{o}(1)\right) \cdot \operatorname{Vol}\left([-L, L]^{d}\right) .
\end{aligned}
$$

The subleading terms in (3.13) also depend on the behavior of $\frac{d \mu}{d \lambda}$ near the origin. For example, let $\hat{K}(\lambda)$ be an indicator, $\hat{K}(\lambda)=\chi_{B}(\lambda), B \subset \mathbb{R}^{d}$. As we have seen above this is equivalent to $\frac{d \mu}{d \lambda}(0)=0$. For simplicity we will assume $d=1$. If $B$ is a union of $m$ disjoint intervals

$$
\begin{aligned}
& \frac{d \mu}{d \lambda}(\lambda)=K(0)-\frac{1}{2 \pi} \int \hat{K}(y) \cdot \hat{K}(y-\lambda) d y= \\
& \frac{1}{2 \pi} \cdot[\operatorname{length}(B)-\operatorname{length}(B \cap(B+\lambda))]= \\
& \frac{m}{2 \pi} \cdot|\lambda| \cdot(1+\bar{o}(1)), \lambda \rightarrow 0,
\end{aligned}
$$

and after more careful evaluation of the asymptotics of $\int_{-L}^{L} \int_{-L}^{L}|K|^{2}(x-$ $y) d x d y=\frac{1}{2 \pi} \cdot \int_{-\infty}^{\infty} \widehat{|K|^{2}}(\lambda) \cdot\left(\frac{2 \sin (L \cdot \lambda)}{\lambda}\right)^{2} d \lambda$ we arrive at

$$
\operatorname{Var}\left(\#_{[-L, L]^{d}}\right)=\frac{m}{\pi^{2}} \log L \cdot(1+\bar{o}(1))
$$

Choosing $m=1, \hat{K}(\lambda)=X_{[-\pi, \pi]}(\lambda)$ one obtains the sine kernel $K(x-y)=$ $\frac{\sin \pi(x-y)}{\pi(x-y)}$. A special role played by the sine kernel can be highlighted by the fact that $\frac{1}{\pi^{2}} \log L$ rate of the growth for $\operatorname{Var}\left(\#_{[-L, L]}\right)$ is the slowest among all translation-invariant kernels $K(x-y)$ corresponding to projectors, $\hat{K}=\chi_{B}$, for which $\inf (B), \sup (B)$ are the density points of $B$ (if $K$ is not a projector it follows from Lemma 6 that the rate of the growth of the variance is linear).

As an example consider $B=\bigsqcup_{n \geq 1}\left[n, n+\frac{1}{n^{\gamma}}\right], \gamma>1$, than one has $\frac{d \mu}{d \lambda} \sim$ $|\lambda|^{1-\frac{1}{\gamma}}$ and $\operatorname{Var}\left(\#_{[-L, L]}\right) \sim L^{\frac{1}{\gamma}}$. More generally, $\frac{d \mu}{d \lambda} \sim|\lambda|^{\alpha}, 0<\alpha<1$, implies $\operatorname{Var}(\#[-L, L]) \sim L^{1-\alpha}$. 


\section{Central Limit Theorem for Counting Func- tion and Empirical Distribution Function of Spacings}

In [CL] Costin and Lebowitz proved the Central Limit Theorem for \# $[-L, L]$ in the case of the sine kernel. The article also contains a remark on p. 71, due to Widom, that the result holds for a larger class of Random Matrix models. In its general form this theorem appeared in [So2].

Theorem 8. Let $E$ be as in (1.1), $\left\{0<K_{t} \leq 1\right\}$ a family of locally trace class operators in $L^{2}(E),\left\{\left(X, \mathcal{B}, P_{t}\right)\right\}$ a family of the corresponding determinantal random point fields in $E$, and $\left\{I_{t}\right\}$ a family of measurable subsets in $E$ such that

$$
\operatorname{Var}_{t} \# I_{t}=\operatorname{Tr}\left(K_{t} \cdot \chi_{I_{t}}-\left(K_{t} \cdot \chi_{I_{t}}\right)^{2}\right) \rightarrow \infty \text { as } t \rightarrow \infty
$$

Then the distribution of the normalized number of particles in $I_{t}$ (with respect to $P_{t}$ ) converges to the normal law, i.e.,

$$
\frac{\#_{t}-\mathbb{E} \#_{I_{t}}}{\sqrt{\operatorname{Var}_{t} \#_{t}}} \stackrel{w}{\longrightarrow} N(0,1)
$$

Remark 12. It was shown in [So2] that the condition (4.1) from Theorem 8 (the growth of the variance) is satisfied for the Airy kernel $\left(K_{t} \equiv K\right.$ from (2.36), $I_{t}$ expanding), the Bessel kernel ( $K_{t} \equiv K$ from (2.37), $I_{t}$ expanding) and for the families of kernels $\left\{K_{n}\right\}$ corresponding to random matrices from the Classical Compact Groups $(\S 2.3 \mathrm{~b}), \S 2.3 \mathrm{c})$ ). In all these cases $\operatorname{Var}_{t} \# I_{t}$ growth logarithmically with respect to $\mathbb{E}_{t} \# I_{t}$.

Remark 13. To construct an example of the kernel $0 \leq K \leq$ Id such that $E \#_{[-n, n]}=\operatorname{Tr} K \cdot \chi_{[-n, n]} \rightarrow \infty$ as $n \rightarrow \infty$, but $\operatorname{Var} \#_{[-n, n]}=\operatorname{Tr}\left(K \cdot \chi_{[-n, n]}-\right.$ $\left.\left(K \cdot \chi_{[-n, n]}\right)^{2}\right)$ stays bounded, consider $\left\{\varphi_{n}(x)\right\}_{n=-\infty}^{\infty}$ satisfying

a) $\operatorname{supp} \varphi_{n} \in(n, n+1)$,

b) $\left\|\varphi_{n}\right\|_{L^{2}}=1$.

Then $K(x, y)=\sum_{n=-\infty}^{\infty}\left(1-\frac{1}{n^{2}+1}\right) \cdot \varphi_{n}(x) \cdot \overline{\varphi_{n}(y)}$ is the desired kernel. Indeed, $\mathbb{E}_{[-n, n]}=\sum_{k=-n}^{n}\left(1-\frac{1}{k^{2}+1}\right) \stackrel{n \rightarrow \infty}{\longrightarrow} \infty, \operatorname{Var} \#_{[-n, n]}=\sum_{k=-n}^{n}\left(1-\frac{1}{k^{2}+1}\right)$. $\frac{1}{k^{2}+1} \rightarrow \sum_{-\infty}^{\infty}\left(1-\frac{1}{k^{2}+1}\right) \cdot \frac{1}{k^{2}+1}<\infty$. From the other side if $0 \leq K \leq$ Id is compact, locally trace class and $\operatorname{Tr} K \cdot \chi_{[-n, n]} \rightarrow+\infty$, then $\operatorname{Tr} K \cdot \chi_{[-n, n]}-$ $\left(K \cdot X_{[-n, n]}\right)^{2} \rightarrow+\infty$. 
The result of Theorem 8 can be generalized to a finite number of intervals. Namely, if $I_{t}^{(1)}, \ldots, I_{t}^{(m)}$ are disjoint subsets such that $\operatorname{Cov}_{t}\left(\#_{I_{t}^{(k)}}, \#_{I_{t}^{(j)}}\right) / V_{t} \rightarrow$ $b_{i j}$ as $t \rightarrow \infty, 1 \leq i, j \leq m$, where $V_{t}$ is some function of $t$ growing to infinity, then the distribution of $\left(\left(\#_{I_{t}^{(k)}}-\mathbb{E}_{t} \#_{I_{t}^{(k)}} / V_{t}^{\frac{1}{2}}\right)_{k=1, \ldots, m}\right.$ converges to the $m$ dimensional centralized normal vector with the covariance matrix $\left(b_{i j}\right)_{1 \leq i, j \leq m}$ (see [So2]).

Finally, we turn our attention to the problem of the global distribution of spacings. Let $E=\mathbb{R}^{d}$ or $\mathbb{Z}^{d},\left\{B_{j}\right\}_{j=1}^{k}$ be some bounded measurable subsets of $E$, and $\left\{n_{j}\right\}_{j=1}^{k}$ be some non-negative integers. We will be interested in the counting statistics of the following type

$$
\eta_{L}\left(B_{1}, \ldots, B_{k} ; n_{1}, \ldots, n_{k}\right):=\#\left(x_{i} \in[-L, L]^{d}: \#_{x_{i}+B_{j}}=n_{j}, j=1, \ldots, k\right)
$$

We can assume without loss of generality that $\left\{B_{j}\right\}$ are disjoint and do not include the origin. If $d=1, k=1, B_{1}=(0, s]$, then $\left.\eta_{L}((0, s]), 0\right)$ is the number of the nearest spacings in $[-L, L]$ greater than $s: \eta_{L}((0, s], 0)=$ $\#\left\{x_{i} \in[-L, L]: x_{i+1}-x_{i}>s\right\}$, and $\left.\eta_{L}((0, s]), n\right)$ is the number of $n$-spacings greater than $s: \eta_{L}((0, s], n)=\#\left\{x_{i} \in[-L, L]: x_{i+n+1}-x_{i}>s\right\}$. In [So1] we proved the convergence in law of the process $\frac{\eta_{L}((0, s], 0)-\mathbb{E} \eta_{L}((0, s], 0)}{L^{\frac{1}{2}}}$ to the limiting Gaussian process in the case $K(x, y)=\frac{\sin \pi(x-y)}{\pi(x-y)}$. Recall that the convergence in law (Functional Central Limit Theorem) implies not only the convergence of the finite-dimensional distributions, but also the convergence of functionals continuous in the appropriate (e.g. locally uniform) topology on the space of sample paths. The proof of the Central Limit Theorem for the finite-dimensional distributions of $\eta_{L}((0, s], 0)$ can be extended essentially word by word to the case of arbitrary, not necessarily translation invariant, kernel $K(x, y)$ and dimension $d \geq 1$, assuming the conditions (4.33), (4.34), (4.35) are satisfied. One can also replace $(0, s]$ by an arbitrary measurable bounded $B \subset E$. For the convenience of the reader we sketch the main ideas of the proof of the finite-dimensional Central Limit Theorem below. Let us fix $B_{1}, \ldots, B_{k} ; n_{1}, \ldots, n_{k}$. We construct a new (called modified) random point field such that $\eta_{L}\left(B_{1}, \ldots B_{k} ; n_{1}, \ldots, n_{k}\right)$ is equal to the number of all particles of the modified random point field in $[-L, L]^{d}$. Namely we keep only those particles of the original r.p.f. for which

$$
\#_{x_{i}+B_{j}}=n_{j}, j=1, \ldots, k,
$$


and throw away the particles for which (4.3) is violated. The modified r.p.f. in general will no longer be a d.r.p.f. What is important is that its correlation functions and cluster functions (see Definition 6 below) can be expressed in terms of the correlation functions of the original determinantal r.p.f. Let us denote by $\rho_{\ell}\left(x_{1}, \ldots, x_{\ell} ; B_{1}, \ldots, B_{k} ; n_{1}, \ldots, n_{k}\right)$ the $\ell$-point correlation function of the modified r.p.f. Suppose that

$$
x_{i} \notin x_{j}+B_{p}, 1 \leq i \neq j \leq \ell, 1 \leq p \leq k .
$$

Then by the inclusion-exclusion principle

$$
\begin{aligned}
& \rho_{\ell}\left(x_{1}, \ldots, x_{\ell} ; B_{1}, \ldots, B_{k} ; n_{1}, \ldots, n_{k}\right)=\sum_{m=0}^{\infty} \frac{(-1)^{m}}{m !} \\
& \int_{\left(x_{1}+B_{1}\right)^{n_{1}} \times \cdots \times\left(x_{1}+B_{k}\right)^{n_{k}}} \int_{\left(x_{\ell}+B_{1}\right)^{n_{1}} \times \cdots \times\left(x_{\ell}+B_{k}\right)^{n_{k}}} \\
& \int_{\left(\left(x_{1}+\bigsqcup_{j=1}^{k} B_{j}\right) \sqcup \ldots \sqcup\left(x_{\ell}+\bigsqcup_{j=1}^{k} B_{j}\right)\right)^{m}} \rho_{\ell+\ell \cdot n+m}\left(x_{1}, \ldots, x_{\ell} ;\right. \\
& \left.x_{11}, \ldots, x_{1 n}, x_{21}, \ldots, x_{2 n}, \ldots, x_{\ell 1}, \ldots, x_{\ell n}, y_{1}, \ldots y_{m}\right) d y_{1} \ldots d y_{m} \\
& d x_{\ell 1} \ldots d x_{\ell n} \ldots d x_{11} \ldots d x_{1 n} \\
& n=n_{1}+\cdots+n_{k} \text {. }
\end{aligned}
$$

If (4.4) is violated then the formula is quite similar, the only difference is that the exponent $n_{j}$ in $\left(x_{i}+B_{j}\right)^{n_{j}}=\left(x_{i}+B_{j}\right) \times \cdots \times\left(x_{i}+B_{j}\right), 1 \leq i \leq$ $\ell, 1 \leq j \leq k$, has to be replaced by $n_{j}-\#\left(1 \leq r \neq i \leq k: x_{r} \in x_{i}+\right.$ $B_{j}$ ). While formulas (4.5) appear to be cumbersome and lengthy, they are nevertheless quite useful for calculating the asymptotics of the moments of $\eta_{L}\left(B_{1}, \ldots, B_{k} ; n_{1}, \ldots n_{k}\right)$. (Of course the assumption that the correlation functions of the original r.p.f. are the determinants is the key here.) Recall the definition of the cluster functions.

Definition 6. The $\ell$-point cluster functions $r_{\ell}\left(x_{1}, \ldots, x_{\ell}\right), \ell=1,2, \ldots$, of a random point field are defined by the formula

$$
r_{\ell}\left(x_{1}, \ldots, x_{\ell}\right)=\sum_{G}(-1)^{m-1}(m-1) ! \cdot \prod_{j=1}^{m} \rho_{\left|G_{j}\right|}\left(\bar{x}\left(G_{j}\right)\right)
$$

where the sum is over all partitions $G$ of $[\ell]=\{1,2, \ldots, \ell\}$ into subsets $G_{1}, \ldots, G_{m}, m=1, \ldots, \ell$, and $\bar{x}\left(G_{j}\right)=\left\{x_{i}: i \in G_{j}\right\},\left|G_{j}\right|=\#\left(G_{j}\right)$. 
The cluster functions are also known in the Statistical Mechanics as the truncated correlated funciton and the Ursell functions. Sometimes in the literature the r.h.s. of $(4.6)$ defines $(-1)^{\ell-1} r_{\ell}$. Correlation functions can be obtained from cluster functions by the inversion formula

$$
\rho_{\ell}\left(x_{1}, \ldots, x_{\ell}\right)=\sum_{G} \prod_{j=1}^{m} r_{\left|G_{j}\right|}\left(\bar{x}\left(G_{j}\right)\right) .
$$

((4.6) is just the Möbius inversion formula to (4.7).) The integrals of $r_{\ell}\left(x_{1}, \ldots, x_{\ell}\right)$ over $[-L, L]^{d} \times \cdots \times[-L, L]^{d}=[-L, L]^{\ell d}$ are closely related to the cumulants $C_{j}(L)$ of the number of particles in $[-L, L]^{d}$ :

$V_{1}(L)=\int_{[-L, L]^{d}} r_{1}\left(x_{1}\right) d x_{1}=C_{1}(L)=\mathbb{E} \#_{[-L, L]^{d}}$

$V_{2}(L) \quad:=\quad \int_{[-L, L]^{d}} \int_{[-L, L]^{d}} r_{2}\left(x_{1}, x_{2}\right) d x_{1}$ $d x_{2}=C_{2}(L)-C_{1}(L)=\operatorname{Var} \#_{[-L, L]^{d}}-\mathbb{E} \#_{[-L, L]^{d}}$,

$V_{3}(L) \quad:=\quad \int_{[-L, L]^{d}} \int_{[-L, L]^{d}} \int_{[-L, L]^{d}}$ $r_{3}\left(x_{1}, x_{2}, x_{3}\right) d x_{1} d x_{2} d x_{3}=C_{3}(L)-3 C_{2}(L)+2 C_{1}(L)$.

In general,

$$
\sum_{n=1}^{\infty} \frac{C_{n}(L)}{n !} z^{n}=\sum_{n=1}^{\infty} \frac{V_{n}(L)}{n !}\left(e^{z}-1\right)^{n}
$$

(see [CL], [So1]). For the determinantal random point fields

$$
r_{\ell}\left(x_{1}, \ldots, x_{\ell}\right)=(-1)^{\ell-1} \sum_{\text {cyclic } \sigma \in S_{\ell}} K\left(x_{1}, x_{2}\right) \cdot K\left(x_{2}, x_{3}\right) \cdot \ldots \cdot K\left(x_{\ell}, x_{1}\right),
$$

where the sum in (4.9) is over all cyclic permutations, and the term written in the body of the sum corresponds to $\sigma=\left(\begin{array}{llll}1 & 2 & 3 & \ldots \ell\end{array}\right)$. One can also rewrite (4.9) as

$$
\begin{aligned}
& r_{\ell}\left(x_{1}, \ldots, x_{\ell}\right)=(-1)^{\ell-1} \cdot \frac{1}{\ell} \sum_{\sigma \in s_{\ell}} K\left(x_{\sigma(1)}, x_{\sigma(2)}\right) . \\
& K\left(x_{\sigma(2)}, x_{\sigma(3)}\right) \ldots \cdot K\left(x_{\sigma(\ell)}, x_{\sigma(1)}\right) .
\end{aligned}
$$

We note that the difference between (4.9) and the formula for $\ell$-point correlation

$$
\rho_{\ell}\left(x_{1}, \ldots, x_{\ell}\right)=\sum_{\sigma \in s_{\ell}}(-1)^{\sigma} K\left(x_{1}, x_{\sigma(1)}\right) \cdot K\left(x_{2}, x_{\sigma(2)}\right) \cdot \ldots \cdot K\left(x, x_{\sigma(\ell)}\right)
$$


is that the summation in (4.9) is only over cyclic permutations. It appears that a relation between $\rho_{\ell}\left(x_{1}, \ldots, x_{\ell} ; B_{1}, \ldots, B_{k} ; n_{1}, \ldots, n_{k}\right)$ and $r_{\ell}\left(x_{1}, \ldots, x_{\ell}\right.$; $B_{1}, \ldots, B_{k} ; n_{1}, \ldots, n_{k}$ ) (at least when (3.19) is satisfied) is of a similar nature.

Lemma 7. Let (4.4) be satisfied. Then

$$
\begin{aligned}
& r_{\ell}\left(x_{1}, \ldots, x_{\ell} ; B_{1}, \ldots, B_{k} ; n_{1}, \ldots, n_{k}\right)=\sum_{m=0}^{\infty} \frac{(-1)^{m}}{m !} \\
& \int_{\left(x_{1}+B_{1}\right)^{n_{1}} \times \cdots \times\left(x_{1}+B_{k}\right)^{n_{k}}} \ldots \int_{\left(x_{\ell}+B_{1}\right)^{n_{1}} \times \cdots \times\left(x_{\ell}+B_{k}\right)^{n_{k}}}^{\longleftarrow} \\
& \int_{\left(\left(x_{1}+\bigsqcup_{j=1}^{k} B_{j}\right) \sqcup \cdots \sqcup{ }^{\prime} x_{\ell}+\bigsqcup_{j=1}^{k} B_{j}\right)^{m}} \rho_{\ell+\ell \cdot n+m, \ell}\left(x_{1}, \ldots, x_{\ell} ; x_{11}, \ldots, x_{1 n},\right. \\
& \left.x_{21}, \ldots, x_{2 n} \ldots, x_{\ell 1}, \ldots, x_{\ell n}, y_{1}, \ldots, y_{m}\right) d y_{1} \ldots d y_{m} \\
& d x_{\ell 1} \ldots d x_{\ell n} \ldots d x_{11} \ldots d x_{1 n},
\end{aligned}
$$

where $\rho_{\ell+\ell \cdot n+m, \ell}$ is defined below in (4.13).

To define $\rho_{\ell+\ell \cdot n+m, \ell}$ recall that $\rho_{\ell+\ell \cdot n+m}\left(x_{1}, \ldots, y_{m}\right)=\sum_{\sigma \in S_{\ell+\ell \cdot n+m}}(-1)^{\sigma}$ $K\left(x_{1}, \sigma\left(x_{1}\right)\right) \cdot \ldots \cdot K\left(y_{m}, \sigma\left(y_{m}\right)\right)$, where $\sigma$ is a permutation on the set of variables $\left(x_{1}, \ldots, x_{\ell}, x_{11}, \ldots, x_{\ell n}, y_{1}, \ldots y_{m}\right)$. We write

$$
\rho_{\ell+\ell \cdot n+m, \ell}\left(x_{1}, \ldots, y_{m}\right)=\sum_{\sigma \in S_{\ell+\ell \cdot n+m}}^{*}(-1)^{\sigma} K\left(x_{1}, \sigma\left(x_{1}\right)\right) \cdot \ldots \cdot K\left(y_{m}, \sigma\left(y_{m}\right)\right) \text {, }
$$

where the summation in $\sum^{*}$ is over the permutations $\sigma$ satisfying the following property:

Let $\tau$ be a multivalued map defined on $\{1, \ldots, \ell\}$ with the values in $\{1, \ldots, \ell\}$ :

$$
\begin{aligned}
& \tau(i)=\left\{j: \sigma\left(\left\{x_{i}, x_{i 1}, \ldots, x_{i n}\right\} \bigsqcup\left(\left\{y_{1}, \ldots, y_{m}\right\} \bigcap\left(x_{i}+\bigsqcup_{p=1}^{k} B_{p}\right)\right)\right)\right. \\
& \left.\bigcap\left(\left\{x_{j}, x_{j 1}, \ldots, x_{j n}\right\} \bigsqcup\left(\left\{y_{1}, \ldots, y_{m}\right\} \bigcap\left(x_{j}+\bigsqcup_{p=1}^{k} B_{p}\right)\right)\right) \neq \emptyset\right\}
\end{aligned}
$$


then for any $1 \leq i, j \leq \ell$ there exists $N=N(i, j)$ such that

$$
\tau^{N}(i) \ni j
$$

Remark 14. The proof of Lemma 7 in the case $d=1, K(x, y)=\frac{\sin \pi(x-y)}{\pi(x-y)}$, $B_{1}=(0, s], n_{1}=0$, was given in $\S 3$ of [So1]. In the general case the argument is absolutely the same. As a corollary of Lemma 7 we obtain

Lemma 8. Let

$$
|K(x, y)| \leq \psi(x-y)
$$

and (4.4) hold for the $\ell$-tuple $\left(x_{1}, \ldots, x_{\ell}\right)$. Then for any $\delta>0$ the following estimate takes place:

$$
\begin{aligned}
& \mid r_{\ell}\left(x_{1}, \ldots, x_{\ell} ; B_{1}, \ldots, B_{k} ; n_{1}, \ldots, n_{k} \mid \leq \operatorname{const}(\ell, \delta) \cdot\right. \\
& \quad \sum_{\text {cyclic } \sigma \in S_{\ell}}\left(\psi\left(x_{2}-x_{1}\right) \cdot \psi\left(x_{3}-x_{2}\right) \cdot \ldots \cdot \psi\left(x_{1}-x_{\ell}\right)\right)^{1-\delta}
\end{aligned}
$$

For the proof of Lemma 8 we refer the reader to [So1] $\S 3$. The key element of the proof is the upper bound on the absolute value of the $m^{\text {th }}$ term in (4.12) by

$$
\begin{gathered}
\operatorname{const}_{1}(n, \ell) \cdot \frac{1}{m !} \operatorname{const}_{2}^{m} \cdot \min \left\{\operatorname{const}_{3}(n, \ell) ;(\ell+\ell n+m) ! \cdot\right. \\
\left.\sum_{\text {cyclic } \sigma \in S_{\ell}}\left(\psi\left(x_{2}-x_{1}\right) \cdot \psi\left(x_{3}-x_{2}\right) \cdot \ldots \cdot \psi\left(x_{1}-x_{\ell}\right)\right)\right\} .
\end{gathered}
$$

If $\psi^{1-\delta} \in L^{2}(E)$ for some $0<\delta<1$, then $\int_{[-L, L]^{d}} \cdots \int_{[-L, L]^{d}} \psi\left(x_{2}-x_{1}\right)^{1-\delta}$. $\ldots \cdot \psi\left(x_{1}-x_{\ell}\right)^{1-\delta} d x_{1} \ldots d x_{\ell} \leq \operatorname{const}(\psi) \cdot \int_{[-L, L]^{d}} \psi(x-y)^{2-2 \delta} d x d y=O\left(L^{d}\right)$, therefore by Lemma 8

$$
\begin{aligned}
& \int_{[-L, L]^{d \ell} \cap(4.4)} r_{\ell}\left(x_{1}, \ldots, x_{\ell} ; B_{1}, \ldots, B_{k} ; n_{1}, \ldots n_{k}\right) \\
& d x_{1} \ldots d x_{\ell}=O\left(L^{d}\right), \ell=1,2, \ldots
\end{aligned}
$$


In particular

$$
\begin{aligned}
& \mathbb{E} \eta_{L}\left(B_{1}, \ldots, B_{k} ; n_{1}, \ldots, n_{k}\right)=V_{1}(L)= \\
& \int_{[-L, L]^{d}} r_{1}\left(x ; B_{1}, \ldots, B_{k} ; n_{1}, \ldots, n_{k}\right) d x=O\left(L^{d}\right)
\end{aligned}
$$

Suppose that one could show

$$
\begin{aligned}
& \operatorname{Var} \eta_{L}\left(B_{1}, \ldots, B_{k} ; n_{1}, \ldots n_{k}\right)=V_{1}(L)+V_{2}(L)= \\
& \int_{[-L, L]^{d}} r_{1}\left(x ; B_{1}, \ldots B_{k} ; n_{1}, \ldots, n_{k}\right) d x+\int_{[-L, L]^{d}} \int_{[-L, L]^{d}} \\
& r_{2}\left(x_{1}, x_{2} ; B_{1}, \ldots, B_{k} ; n_{1}, \ldots n_{k}\right) d x_{1} d x_{2}=\mathrm{const} \cdot L^{d}(1+\bar{o}(1)), \\
& \int_{[-L, L]^{\ell d} \backslash(4.4)} r_{\ell}\left(x_{1}, \ldots, x_{\ell} ; B_{1}, \ldots, B_{k} ; n_{1}, \ldots n_{k}\right) d x_{1} \ldots d x_{\ell} \\
& =\bar{o}\left(L^{\frac{\ell d}{2}}\right), \ell>2 .
\end{aligned}
$$

Since the $\ell^{t h}$ cumulant of $\eta_{L}$ is a linear combination of $V_{i}(L), i=1,2, \ldots \ell$ (see (4.8)), the estimates (4.18)-(4.21) would imly that the $\ell^{\text {th }}$ cumulant of $\eta_{L}$ is const $\cdot L \cdot(1+\bar{o}(1))$ for $\ell=2$ and grows slower than $L^{\frac{\ell d}{2}}$ for $\ell>2$. This in turn would imply that while the second cumulant of $\frac{\eta_{L}-\mathbb{E} \eta_{L}}{\sqrt{\operatorname{Var} \eta_{L}}}$ is 1 , all the other cumulants of $\frac{\eta_{L}-\mathbb{E} \eta_{L}}{\sqrt{\operatorname{Var} \eta_{L}}}$ go to zero as $L \rightarrow+\infty$. The last statement is equivalent to the statement that the moments of $\frac{\eta_{L}-\mathbb{E} \eta_{L}}{\sqrt{\operatorname{Var} \eta_{L}}}$ converge to the moments of the normal distribution, and in particular

$$
\frac{\eta_{L}-\mathbb{E} \eta_{L}}{\sqrt{\operatorname{Var} \eta_{L}}} \stackrel{w}{\longrightarrow} N(0,1) .
$$

Of course the devil is in the details. It turns out that there is no nice extension of the formulas $(4.12),(4.13)$ to the case when (4.4) is not satisfied. Below we show how one can overcome these difficulties in the case of $\eta_{L}(B ; 0)$ (i.e. $\left.k=1, n_{1}=0\right)$. We introduce the centralized $\ell$-point correlation functions by the formula

$$
\rho_{\ell}^{(c)}\left(x_{1}, \ldots, x_{\ell}\right)=\sum_{G}^{* *} \prod_{j=1}^{m} r_{\left|G_{j}\right|}\left(\bar{x}\left(G_{j}\right),\right.
$$


where $\sum^{* *}$ is the sum over all partitions $G=\left\{G_{1}, \ldots, G_{m}\right\}, m=1,2, \ldots$ of $\{1, \ldots, \ell\}$ into two- and more element subsets (i.e. $\left|G_{j}\right|>1, j=1, \ldots, m$ ). It follows from (4.7), (4.22) that

$$
\begin{aligned}
& \rho_{\ell}^{(c)}\left(x_{1}, \ldots, x_{\ell}\right)=\rho_{\ell}\left(x_{1}, \ldots, x_{\ell}\right)+\sum_{p=1}^{\ell}(-1)^{p} \sum_{1 \leq i_{1}<\cdots<i_{p} \leq \ell} \prod_{s=1}^{p} \\
& \rho_{1}\left(x_{i_{s}}\right) \cdot \rho_{\ell-p}\left(\left(x_{1}, \ldots, x_{\ell}\right) \backslash\left(x_{i_{1}}, \ldots, x_{i_{p}}\right)\right)=\rho_{\ell}\left(x_{1}, \ldots, x_{\ell}\right)- \\
& \sum_{p=1}^{\ell} \sum_{1 \leq i_{1}<\cdots<i_{p} \leq \ell} \prod_{s=1}^{p} \rho_{1}\left(x_{i_{s}}\right) \cdot \rho_{\ell-p}^{(c)}\left(\left(x_{1}, \ldots, x_{\ell}\right) \backslash\left(x_{i_{1}}, \ldots, x_{i_{p}}\right)\right) .
\end{aligned}
$$

Let us denote by $M_{(\ell)}^{(c)}(L)$ the integral of the centralized $\ell$-point correlation function of the modified random point field over $[-L, L]^{\ell d}$,

$$
M_{(\ell)}^{(c)}(L)=\int_{[-L, L]^{d}} \cdots \int_{[-L, L]^{d}} \rho_{\ell}^{(c)}\left(x_{1}, \ldots, x_{\ell} ; B_{1} ; 0\right) d x_{1} \ldots d x_{\ell} .
$$

We have

$$
\begin{aligned}
& \sum_{\ell=0}^{\infty} \frac{t^{\ell}}{\ell !} \mathbb{E}\left(\eta_{L}-\mathbb{E} \eta_{L}\right)^{\ell}=e^{-t \mathbb{E} \eta_{L}} \cdot \sum_{\ell=0}^{\infty} \frac{t^{\ell}}{\ell !} \mathbb{E} \eta_{L}^{\ell}=e^{-t \mathbb{E} \eta_{L}} \cdot \sum_{\ell=0}^{\infty} \frac{\left(e^{t}-1\right)^{\ell}}{\ell !} \\
& \mathbb{E} \eta_{L} \cdot\left(\eta_{L}-1\right) \cdot \ldots \cdot\left(\eta_{L}-\ell+1\right)=e^{-t \mathbb{E} \eta_{L}} \cdot e^{\left(e^{t}-1\right) \mathbb{E} \eta_{L}} \cdot \\
& \sum_{\ell=0}^{\infty} \frac{\left(e^{t}-1\right)^{\ell}}{\ell !} M_{(\ell)}^{(c)}(L)
\end{aligned}
$$

If we can show that

$$
M_{\ell}^{(c)}(L)=\left\{\begin{array}{l}
(2 n-1) ! ! \cdot \text { const }_{1}^{n} \cdot L^{n d} \cdot(1+\bar{o}(1)) \text { for } \ell=2 n, \\
\bar{o}\left(L^{\frac{\ell d}{2}}\right) \text { for } \ell=2 n+1,
\end{array}\right.
$$

and

$$
\mathbb{E} \eta_{L}=\text { const }_{2} \cdot L^{d} \cdot(1+\bar{o}(1))
$$

then (4.25) implies

$\mathbb{E}\left(\eta_{L}-\mathbb{E} \eta_{L}\right)^{\ell}=\left\{\begin{array}{l}(2 n-1) ! ! \cdot\left(\text { const }_{1}+\text { const }_{2}\right)^{n} \cdot L^{n d} \cdot(1+\bar{o}(1)) \text { for } \ell=2 n, \\ \bar{o}\left(L^{\frac{\ell d}{2}}\right) \text { for } \ell=2 n+1,\end{array}\right.$ 
and

$$
\frac{\eta_{L}-\mathbb{E} \eta_{L}}{L^{\frac{d}{2}}} \stackrel{w}{\longrightarrow} N\left(0, \text { const }_{1}+\text { const }_{2}\right)
$$

One can in principle calculate $M_{\ell}^{(c)}(L)$ from (4.12), (4.13). Indeed, if

$$
x_{i}-x_{j} \notin B
$$

(we remark that (4.29) is exactly (4.4) written in the case $k=1, n_{1}=0$ ), then the expression for $\rho_{\ell}^{(c)}\left(x_{1}, \ldots, x_{\ell} ; B ; 0\right)$ can be obtained from (4.22), (4.12), (4.13). Otherwise $\rho_{\ell}\left(x_{1}, \ldots, x_{\ell} ; B ; 0\right)=0$, and (4.23) implies

$$
\begin{aligned}
& \rho_{\ell}^{(c)}\left(x_{1}, \ldots, x_{\ell} ; B ; 0\right)=\sum_{p=1}^{\ell}(-1)^{p} \cdot \sum_{1 \leq i_{1}<\cdots<i_{p} \leq \ell} \prod_{s=1}^{p} \\
& r_{1}\left(x_{i_{s}} ; B ; 0\right) \cdot \rho_{\ell-p}\left(\left(x_{1}, \ldots, x_{\ell}\right) \backslash\left(x_{i_{1}}, \ldots, x_{i_{p}}\right)\right) .
\end{aligned}
$$

If for an $(\ell-p)$-tuple $\left(x_{1}, \ldots, x_{\ell}\right) \backslash\left(x_{i_{1}}, \ldots, x_{i_{p}}\right)$ the condition (4.29) is not satisfied, then the corresponding term $\rho_{\ell-p}\left(\left(x_{1}, \ldots, x_{\ell}\right) \backslash\left(x_{i_{1}}, \ldots, x_{i_{p}}\right)\right)$ in (4.20) is zero. If $(4.29)$ is satisfied for $\left(x_{1}, \ldots, x_{\ell}\right) \backslash\left(x_{i_{1}}, \ldots, x_{i_{p}}\right)$, then we iterate (4.23) again

$$
\begin{aligned}
& \rho_{\ell-p}\left(\left(x_{1}, \ldots, x_{\ell}\right) \backslash\left(x_{i_{1}}, \ldots, x_{i_{p}}\right)\right)= \\
& \left.\rho_{\ell-p}^{(c)}\left(\left(x_{1}, \ldots, x_{\ell}\right)\right) \backslash\left(x_{i_{1}}, \ldots, x_{i_{p}}\right)\right)+\sum \ldots
\end{aligned}
$$

We claim

Lemma 9. Let the condition (4.29) be not satisfied for the $\ell$-tuple $\left(x_{1}, \ldots, x_{\ell}\right)$. Then

$$
\rho_{\ell}^{(c)}\left(x_{1}, \ldots, x_{\ell} ; B ; 0\right)=\sum_{\emptyset \subseteq D \subset\{1, \ldots, \ell\}} C_{D} \cdot \prod_{i \notin D} r_{1}\left(x_{i} ; B ; 0\right) \cdot \rho_{|D|}^{(c)}(\bar{x}(D)),
$$

where

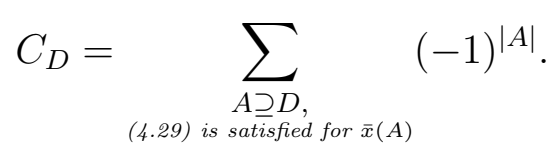


In particular $C_{D}=0$ if (4.29) is not satisfied for $\bar{x}(D)$ or if there exists $1 \leq i \leq \ell, i \notin D$, such that for any $1 \leq j \leq \ell x_{i}-x_{j} \notin B \cup(-B)$.

Proof easily follows from the above arguments.

Theorem 9. Let $(X, B, P)$ be a determinantal random point field with the kernel

$$
|K(x, y)| \leq \psi(x-y)
$$

where $\psi$ is a bounded non-negative function such that $\psi \cdot\left(\log \left(\frac{\psi+1}{\psi}\right)\right)^{n} \in L^{2}(E)$ for any $n>0$. Let for $\eta_{L}(B ; 0)=\#\left(x_{i} \in[-L, L]^{d}: \#\left(x_{i}+B\right)=0\right)$ we have

$$
\operatorname{Var} \eta_{L}(B ; 0)=\sigma^{2} \cdot L^{d} \cdot(1+\overline{0}(1))
$$

Then the Central Limit Theorem holds:

$$
\frac{\eta_{L}(B, 0)-\mathbb{E} \eta_{L}(B ; 0)}{L^{\frac{d}{2}}} \stackrel{w}{\longrightarrow} N\left(0, \sigma^{2}\right) .
$$

Remark 15. If $\operatorname{Cov}\left(\eta_{L}\left(B_{i} ; 0\right), \eta_{L}\left(B_{j} ; 0\right)\right)=b_{i j} \cdot L^{d} \cdot(1+\overline{0}(1)), 1 \leq i, j \leq p$, then

$$
\left(\frac{\eta_{L}\left(B_{i} ; 0\right)-\mathbb{E} \eta_{L}\left(B_{i} ; 0\right)}{L^{\frac{d}{2}}}\right)_{1 \leq i \leq p} \stackrel{w}{\longrightarrow} N\left(0,\left(b_{i j}\right)_{1 \leq i, j \leq p}\right) .
$$

Recall that

$$
\begin{aligned}
& \operatorname{Cov}\left(\eta_{L}\left(B_{i} ; 0\right) ; \eta_{L}\left(B_{j} ; 0\right)\right)=\mathbb{E}\left(\eta_{L}\left(B_{i} ; 0\right)-\mathbb{E} \eta_{L}\left(B_{i}, 0\right)\right) \cdot\left(\eta_{L}\left(B_{j} ; 0\right)-\right. \\
& \left.\mathbb{E} \eta_{L}\left(B_{j} ; 0\right)\right)=\int_{\cap\left\{x_{1}-x_{2} \notin B_{i} \cup\left(-B_{j}\right)\right\}}\left(\sum_{m=0}^{\infty} \frac{(-1)^{m}}{m !} \cdot \int_{\left(\left(x_{1}+B_{i}\right) \sqcup\left(x_{2}+B_{j}\right)\right)^{m}}\right. \\
& \left.\rho_{2+m, 2}\left(x_{1}, x_{2} ; y_{1}, \ldots, y_{m}\right) d y_{1} \ldots d y_{m}\right) d x_{1} d x_{2}-\int_{[-L, L]^{d}} r_{1}\left(x_{1} ; B_{i} ; 0\right) \text {. } \\
& \int_{\left(x_{1}+B_{i}\right) \cup\left(x_{1}-B_{j}\right)} r_{1}\left(x_{2} ; B_{j} ; 0\right) d x_{2} d x_{1}+\int_{[-L, L]^{d}} r_{1}\left(x ; B_{1} \cup B_{2}, 0\right) d x
\end{aligned}
$$

Remark 16. Lemma 8 suggests slightly more restrictive condition on $\psi$, namely $\psi^{1-\delta} \in L^{2}(E)$ for some $0<\delta<1$. However, looking at the proof of Lemma 6 one immediately realizes that it is possible to replace $\psi^{1-\delta}$ in (4.17) by $\psi \cdot\left(\log \left(\frac{\psi+1}{\psi}\right)\right)^{n}$ with $n>3 \ell$. 
Proof of Theorem 9. It follows from (4.25)-(4.28) that it is enough to show

$$
\begin{aligned}
& \int_{[-L, L]^{2 n d}} \rho_{2 n}^{(c)}\left(x_{1}, \ldots, x_{2 n} ; B ; 0\right) d x_{1} \ldots d x_{2 n}= \\
& (2 n-1) ! ! \cdot\left(\int_{\substack{[-L, L]^{2 d} \cap \\
\{x-y \notin B \cap(-B)\}}} r_{2}(x, y ; B ; 0) d x d y-\int_{[-L, L]^{d}}\right. \\
& \left.r_{1}(x ; B ; 0) \int_{(x+B) \cup(x-B)} r_{1}(y ; B ; 0) d y d x\right)^{n}+\bar{o}\left(L^{n d}\right), \\
& n=1,2, \ldots, \\
& \int_{[-L, L]^{(2 n+1) d}} \rho_{2 n+1}^{(c)}\left(x_{1}, \ldots, x_{2 n+1} ; B ; 0\right) d x_{1} \ldots d x_{2 n+1}=\bar{o}\left(L^{\frac{2 n+1}{2} d}\right), \\
& n=1,2, \ldots
\end{aligned}
$$

\section{Lemma 10.}

$$
\begin{gathered}
\int_{[-L, L]^{2 n d} \cap(4.29)} \rho_{2 n}^{(c)}\left(x_{1}, \ldots, x_{2 n} ; B ; 0\right) d x_{1} \ldots d x_{2 n}= \\
(2 n-1) ! ! \cdot\left(\int_{\substack{\{-L, L]^{2 d} \cap \\
\{x-y \notin B \cup(-B)\}}} r_{2}(x, y ; B ; 0) d x d y\right)^{n}+\bar{o}\left(L^{n d}\right), \\
\int_{[-L, L]^{(2 n+1) d} \cap(4.29)} \rho_{2 n+1}^{(c)}\left(x_{1}, \ldots, x_{2 n+1} ; B ; 0\right) d x_{1} \ldots d x_{2 n+1}=\bar{o}\left(L^{\frac{2 n+1}{2} d}\right) .
\end{gathered}
$$

Recall that all $r_{\ell}\left(x_{1}, \ldots, x_{\ell} ; B ; 0\right)$ are bounded functions (see (4.17)). Let us rewrite (4.22) as

$$
\rho_{\ell}^{(c)}\left(x_{1}, \ldots, x_{\ell}\right)=\sum_{G}^{\prime} \prod_{j=1}^{m} r_{\left|G_{j}\right|}\left(\bar{x}\left(G_{j}\right)\right)+\sum_{G}^{\prime \prime} \prod_{j=1}^{m} r_{\left|G_{j}\right|}\left(\bar{x}\left(G_{j}\right)\right),
$$

where $\sum^{\prime}$ is the sum over all partitions of $\{1, \ldots, \ell\}$ into pairs, and $\sum^{\prime \prime}$ is the sum over all other two- and more element partitions. Let $\ell$ be even, 
$\ell=2 n$. Integrating $\sum_{G}^{\prime}$ over $[-L, L]^{2 n d} \cap(4.29)$ we obtain exactly the r.h.s. of $(4.38)$ (there are $(2 n-1)$ !! partitions of $\{1, \ldots, 2 n\}$ into twoelement sets). It follows from (4.17) and the estimate below Lemma 8 that $\int_{[-L, L]^{\ell d}}\left|r_{\ell}\left(x_{1}, \ldots, x_{\ell} ; B ; 0\right)\right| d x_{1} \ldots d x_{\ell}=\underline{O}\left(L^{d}\right)$. Therefore the integral of $\sum_{G}^{\prime \prime}$ over $[-L, L]^{2 n d} \cap(4.29)$ is $\bar{o}\left(L^{n d}\right)$. The formula (4.39) can be proven in the same way.

To estimate

$$
\int_{[-L, L]^{2 n d} \backslash(4.29)} \rho_{2 n}^{(c)}\left(x_{1}, \ldots, x_{2 n} ; B ; 0\right) d x_{1} \ldots d x_{2 n}
$$

we introduce the equivalence relation on $\left\{x_{1}, \ldots, x_{2 n}\right\}$ by calling $x_{i}, x_{j}$ "neighbors" if there exists a sequence of indices $1 \leq i_{0}, i_{1}, \ldots, i_{u} \leq 2 n, 1 \leq u \leq 2 n$, such that $i_{0}=i, i_{u}=j$, and $x_{i_{s+1}}-x_{i_{s}} \in B \cup(-B), s=0, \ldots, u-1$. We claim that the contributions of order $O\left(L^{n d}\right)$ appear in (4.40) only from such sets of $\left(x_{1}, \ldots x_{2 n}\right)$ where each equivalence class of "neighbors" has either one or two indices. Consider for example the case when we have $k$ two-element classes $\left\{x_{1}, x_{2}\right\}, \ldots,\left\{x_{2 k-1}, x_{2 k}\right\}$ and $2 n-2 k$ one-element equivalence classes $\left\{x_{2 k+1}\right\}, \ldots,\left\{x_{2 n}\right\}$. Similarly to the calculations on pp. 596-597 of [So1] we verify that the integral of $\rho_{2 n}^{(c)}\left(x_{1}, \ldots, x_{2 n} ; B ; 0\right)$ over the subset of $[-L, L]^{2 n d}$ corresponding to the above partition is equal to

$$
\begin{aligned}
& (2 n-2 k-1) ! ! \cdot\left(-\int_{[-L, L]^{d}} r_{1}(x ; B ; 0) \int_{(x+B) \cup(x-B)} r_{1}(y ; B ; 0) d y d x\right)^{k} \\
& \cdot\left(\int_{\substack{[-L, L]^{2 n} \cap \\
\{x-y \notin B \cap(-B)\}}} r_{2}(x, y ; B ; 0) d x d y\right)^{n-k}+\bar{o}\left(L^{n d}\right) .
\end{aligned}
$$

After the summation over all partitions into one- and two-element equivalence classes of "neighbors" (we remark that (4.38) corresponds to the partition into singletons), we obtain exactly (4.36). It follows from Lemma 7 and (4.17) that all other partitions into the equivalence classes give negligible contributions. (4.37) can be proven in a similar fashion.

The conditions of Theorem 9 are very unrestrictive in the case of translation invariant kernels. The covariance function of the limiting Gaussian process w. $\lim \frac{\eta_{L}((0, \bar{s}], 0)-\mathbb{E} \eta_{L}((0, \bar{s}] ; 0)}{L^{d / 2}}$ is then given by the $d$-dimensional analogues of the formulas (37), (38), (26) from [So1] (of course one has to replace $\frac{\sin \pi(x-y)}{\pi(x-y)}$ by $\left.K(x-y)\right)$. Here and below we denote by $(0, \bar{s}]$ the rectangle 
$\left(0, s_{1}\right] \times \cdots \times\left(0, s_{d}\right], \bar{s}=\left(s_{1}, \ldots, s_{d}\right)$. In particular, if $K(x)$ is continuously differentiable the limiting Gaussian process is Hölder-continuous with any exponent less than $\frac{1}{2}$. Among other characteristics of the modified random point field (with respect to $B=(0, \bar{s}], n=0)$ one may be interested in the spectral measure of the restriction of the group $\left\{U^{t}\right\}$ to the subspace of the centralized linear statistics. We shall denote the spectral measure by $\mu^{(s)}(d \lambda)$. Recall that the spectral measure $\mu^{(0)}(d \lambda)=\mu(d \lambda)$ of the original determinantal random point field is given by (3.12). In particular, for the sine kernel

$$
\frac{d \mu}{d \lambda}= \begin{cases}\frac{|\lambda|}{2 \pi}, & |\lambda| \leq 2 \pi \\ 1, & |\lambda|>2 \pi\end{cases}
$$

After lengthy, but rather straightforward calculations one can obtain that in the case of the sine kernel :

$$
\frac{d \mu^{(s)}}{d \lambda}=\frac{\pi^{2} s^{3}}{9}+\frac{|\lambda|}{2 \pi} \cdot\left(1-\frac{4}{3} \pi^{2} s^{3}\right)+O\left(s^{4}\right)+O\left(|\lambda| \cdot s^{4}\right)+O\left(\left|\lambda^{2}\right| \cdot s^{2}\right)
$$

We note that $\frac{d \mu^{(s)}}{d \lambda}(0) \neq 0$ if $s \neq 0, s$ small, which is consistent with Var $\eta_{L}((0, s] ; 0) \sim L$. For the proof of the Functional Central Limit Theorem we refer the reader to pp. 577, 598-600 of [So1]. Suppose that

$$
\left.L^{-d} \frac{\partial}{\partial s} \eta_{L}((0, \bar{s}] ; 0)\right), L^{-d} \frac{\partial}{\partial s} \operatorname{Cov}\left(\eta_{L}((0, \bar{s}] ; 0) ; \eta_{L}((0, \bar{t}] ; 0)\right)
$$

are uniformly bounded in $L, \bar{s}, \bar{t}$, where $\bar{s}, \bar{t}$ belong to compact subsets of $\mathbb{R}_{+}^{d}\left(\mathbb{Z}_{+}^{d}\right)$. By smoothing with a $C^{\infty}$ approximate $\delta$-function one can construct a continuous approximation $\tilde{\eta}_{L}((0, \bar{s}] ; 0)$ such that $\left|\tilde{\eta}_{L}((0, \bar{s}] ; 0)-\eta_{L}((0, \bar{s}] ; 0)\right| \leq$ 1. As a result

$$
\frac{\tilde{\eta}_{L}((0, \bar{s}] ; 0)-\mathbb{E} \tilde{\eta}_{L}((0, \bar{s}] ; 0)}{L^{\frac{d}{2}}}
$$

is a random continuous function in $\bar{s}$, and

$$
\left|\frac{\tilde{\eta}_{L}((0, \bar{s}] ; 0)-\mathbb{E} \tilde{\eta}_{L}((0, \bar{s}] ; 0)}{L^{\frac{d}{2}}}-\frac{\eta_{L}((0, \bar{s}] ; 0)-\mathbb{E} \eta_{L}((0, \bar{s}] ; 0)}{L^{\frac{d}{2}}}\right| \leq \frac{2}{L^{\frac{d}{2}}}
$$

The distribution of the random process $\frac{\tilde{\eta}_{L}((0, \bar{s}] ; 0)-\mathbb{E} \tilde{\eta}_{L}((0, \bar{s}] ; 0)}{L^{\frac{d}{2}}}$ defines a probability measure on $C\left([0, \infty)^{d}\right)$. By the convergence in law of random processes we mean the weak convergence of the induced probability measures 
on $C\left([0, \infty)^{d}\right)$ (see $[\mathrm{B}]$, in general one can consider different spaces of sample paths, e.g. the space of càdlàg functions, instead of the space of continuous functions).

Theorem 10. Let the condition (4.33), (4.34), (4.35), (4.43) be satisfied. Then the random process

$$
\frac{\tilde{\eta}_{L}((0, \bar{s}] ; 0)-\mathbb{E} \tilde{\eta}_{L}((0, \bar{S}] ; 0)}{L^{\frac{1}{2}}}
$$

converges in law to the limiting Gaussian process.

\section{References}

[A] N.I. Akhiezer, The Classical Moment Problem and Some Related Questions in Analysis, Hafner Publishing Co., New York, 1965.

[AF $] \quad$ A. Alastuey and P.J. Forrester, Correlations in two-component log-gas system, J. Stat. Phys. 81, Nos. 3/4, 579-627, 1995.

[AL] A. Alastuey and J.L. Lebowitz, The two-dimensional onecomponent plasma in an inhomogeneous background - exact results, J. Phys. (France), 45, 1859-1874, 1984.

[AD] D. Aldous and P. Diaconis, Longest increasing subsequences: From patience sorting to the Baik-Deift-Johansson theorem, Bull. Amer. Math. Soc. (N.S.), 36, No. 4, 413-432, 1999.

[AM] M. Adler, P. van Moerbeke, The spectrum of coupled random matrices, Ann. of Math. 149, No. 3, 921-976, 1999.

[AvSS] J.E. Avron, R. Seiler, and B. Simon, Charge deficiency, charge transport and comparison of dimensions, Commun. Math. Phys. 159, 399-422, 1994.

[BDJ1] J. Baik, P.A. Deift, and K. Johansson, On the distribution of the length of the longest increasing subsequence of random permutations, J. Amer. Math. Soc., 12, 1119-1178, 1999. 
[BDJ2] J. Baik, P.A. Deift, and K. Johansson, On the distribution of the length of the second row of a Young diagram under Plancherel measure, available via http://xxx.lanl.gov/abs/math/9901118.

[BR1] J. Baik and E.M. Rains, Algebraic aspects of increasing subsequences, available via http://xxx.lanl.gov/abs/math/9905083.

[BR2] J. Baik and E.M. Rains, The asymptotics of monotone subsequences of involutions, available via http://xxx.lanl.gov/abs/math/9905084.

[BR3] J. Baik and E.M. Rains, Symmetrized random permutations, available via http://xxx.lanl.gov/abs/math/9910019.

[B] P. Billingsley, Convergence of Probability Measures, John Wiley \& Sons, New York, 1968.

[BI] P. Bleher and A. Its, Semiclassical asymptotics of orthogonal polynomials, Riemann-Hilbert problem and universality in the matrix model, Ann. of Math. 150, No. 1, 185-266, 1999.

[Bor] A. Borodin, Longest increasing subsequences of random colored permutations, available via http://xxx.lanl.gov/abs/math/9902001.

[BO1] A. Borodin and G. Olshanski, Distributions on partitions, point processes and the hypergeometric kernel, to appear in Commun. Math. Phys., available via http://xxx.lanl.gov/abs/math/9904010.

[BO2] A. Borodin and G. Olshanski, $Z$-measures on partitions, Robinson-Schensted-Knuth correspondence, and $\beta=2$ random matrix ensembles, available via http://xxx.lanl.gov/abs/math/9905189.

[BO3] A. Borodin and G. Olshanski, Point processes and the infinite symmetric group, Math. Res. Lett. 5, 799-816, 1998.

[BOO] A. Borodin, A. Okounkov, and G. Olshanski, On asymptotics of Plancherel measures for symmetric groups, available via http://xxx.lanl.gov/abs/math/9905032. 
[Br] B.V. Bronk, Exponential ensemble for random matrices, $J$. Math. Phys. 6, 228-237, 1965.

[Ch] T.S. Chihara, An Introduction to Orthogonal Polynomials, Gordon and Breach, New York, 1978.

[CFS] I.P. Cornfeld, S.V. Fomin, and Ya.G. Sinai, Ergodic Theory, Springer-Verlag, New York, 1982.

[CJ1] F. Cornu and B. Jancovici, On the two-dimensional Coulomb gas, J. Stat. Phys. 49, 33-56, 1987.

[CJ2] F. Cornu and B. Jancovici, Two-dimensional Coulomb systems - A larger class of colvable models, Europhys. Lett. 5, 125-128, 1988.

[CJ3] F. Cornu and B. Jancovici, Electrical double layer: A solvable model, J. Chem. Phys.. 90, 2444-2452, 1989.

[CL] O. Costin and J. Lebowitz, Gaussian fluctuations in random matrices, Phys. Rev. Lett. 75, No. 1, 69-72, 1995.

[DE] P.Diaconis, S.N. Evans, Immanants and finite point processes, to appear in J. Comb. Th. A, .

[DS] P.Diaconis, M.Shahshahani, On the eigenvalues of random matrices, Studies in Appl. Probab., Essays in honour of Lajos Takacs, J.Appl. Prob., Special Vol. 31 A , 49-62, 1994.

[DVJ] D.J. Daley and D. Vere-Jones, An Introduction to the Theory of Point Processes, Springer-Verlag, New York, 1988.

[De] P. Deift, Orthogonal Polynomials and Random Matrices: A Riemann-Hilbert Approach, Courant Lecture Notes in Mathematics, 3, New York, 1999.

[DKMLVZ] P. Deift, T. Kriecherbauer, K.T.-R. McLaughlin, S. Venakides, and X. Zhou, Uniform asymptotics for polynomials orthogonal with respect to varying exponential weights and applications to universality questions in random matrix theory, Commun. Pure Appl. Math,, 52, No. 11, 1335-1425, 1999.. 
[DFGIL] F. Di Francesco, M. Gaudin, C. Itzykson, and F. Lesage, Laughlin wave-functions, Coulomb gases and expansions of the discriminant, Int. J. Mod. Phys. A, 9, 4257-4351, 1994.

[D1-D3] F.J. Dyson, Statistical theory of the energy levels of complex system, I, II, III, J. Math. Phys. 3, 140-175, 1962.

[E] A. Erdelyi (ed.), Higher Transcendental Functions, vols. 1-2, McGraw-Hill, New York, 1953.

[EM] B. Eynard and M.L. Mehta, Matrices coupled in a chain. I. Eigenvalue correlations, J. Physics A, 31, No. 19, 4449-4456, 1998.

[Fe] W. Feller, An Introduction to Probability Theory and Its Applications, vol. II, John Wiley \& Sons, New York, 1966.

[F] P.J. Forrester, The spectrum edge of random matrix ensembles, Nucl. Phys. B402, 709-728, 1993.

[FJ1] P.J. Forrester and B. Jancovici, Two-dimensional onecomponent plasma in quadrupolar field, Int. J. Mod. Phys. A, 11, 941-949, 1996.

[FJ2] P.J. Forrester and B. Jancovici, On the averge distance between particles in the 2-dimensional 2-component plasma, J. Stat. Phys. 69, 163-178, 1992.

[Fu] W. Fulton, Young Tableaux, Cambridge University Press, 1997.

[FKS1] Ya. V. Fyodorov, B.A. Khoruzhenko, and H.-J. Sommers, Almost Hermitian random matrices: Crossover from WignerDyson to Ginibre eigenvalue statistics, Phys. Rev. Lett. 79. No. 4, 557-560, 1997.

[FKS2] Ya. V. Fyodorov, B.A. Khoruzhenko, and H.-J. Sommers, Universality in the random matrix spectra in the regime of weak non-Hermiticity, Ann. Inst. Henri Poincaré, 68, No. 4, 449489, 1998.

[Ga] M. Gaudin, Critical isoterm of a lattice plasma, J. Phys. (France), 46, 1027-1042, 1985. 
[Gin] J. Ginibre, Statistical ensembles of complex, quaternion and real matrices, J. Math. Phys. 6, 440-449, 1965.

[Gir1] V. Girko, Circle law, Theor. Prob. Appl. 29, 694-, 1985.

[Gir2] V. Girko, Elliptic law, Theor. Prob. Appl. 30, 677-, 1986.

[GK] I. Gohberg and M. Krein, Introduction to the theory of linear non-self adjoint operators, Translations AMS, 18, 1969.

[IL] I.A. Ibragimov and Ju. V. Linnik, Independent and Stationary Random Variables, Wolters-Noordhoff Publishing, Groningen, $1971 .$.

[ITW] A.R. Its, C.A. Tracy, and H. Widom, Random words, Toeplitz determinants and integrable systems. I., available via http://xxx.lanl.gov/abs/math/9909169.

[Ja1] B. Jancovici, Exact results for the two-dimensional onecomponent plasma, Phys. Rev. Lett. 46, 386-388, 1981.

[Ja2] B. Jancovici, Classical Coulomb systems near a plane wall. 1, J. Stat. Phys. 28, 43-65, 1982.

[Jo1] K. Johansson, On random matrices from the compact classical groups, Ann. of Math. 145, 519-545, 1997.

[Jo2] K. Johansson, Shape fluctuations and random matrices, to appear in Commun. Math. Phys., available via http://xxx.lanl.gov/abs/math/9903134.

[Jo3] K. Johansson, Discrete orthogonal polynomial ensembles and the Plancherel measure, available via http://xxx.lanl.gov/abs/math/9906120.

[Jo4] K. Johansson, Universality of local eigenvalue correlations in certain Hermitian Wigner matrices, preprint, 1998.

[KS] N. Katz and P. Sarnak, Random Matrices, Frobenius Eigenvalues and Monodromy, AMS Colloq. Series, 1998. 
[KOV] S. Kerov, G. Olshanski, and A. Vershik, Harmonic analysis on the infinite symmetric group. A deformation of the regular representation, C.R. Acad. Sci. Paris Sér. I Math, 316, No. 8, 773-778, 1993.

[K] A.N. Kolmogorov, The Foundations of the Theory of Probability, 2nd ed., Chelsea Publishing Co., New York, 1956.

$[\mathrm{Ku}] \quad$ G. Kuperberg, Random words, quantum statistics, central limits, random matrices, available via http://xxx.lanl.gov/abs/math/9909104.

[L1] A. Lenard, Correlation functions and the uniqueness of the state in classical statistical mechanics, Commun. Math. Phys. 30, 35-44, 1973.

[L2] A. Lenard, States of classical statistical mechanical system of infinitely many particles I, Arch. Rational Mech. Anal. 59, 219239, 1975.

[L3] A. Lenard, States of classical statistical mechanical system of infinitely many particles II, Arch. Rational Mech. Anal. 59, 240-256, 1975.

[L4] A. Lenard, Momentum distribution in the ground state of the one-dimensional system of impenetrable boson, J. Math. Phys. 5, 930-943, 1964.

[L5] A. Lenard, One-dimensional impenetrable bosons in thermal equilibrium, J. Math. Phys. 7, 1268-1272, 1966.

[Ma] O. Macchi, The coincidence approach to stochastic point processes, Adv. Appl. Prob. 7, 83-122, 1975.

[Mac] I.G. Macdonald, Symmetric Functions and Hall Polynomials, 2nd ed., Oxford University Press, 1995.

[Me] M.L. Mehta, Random Matrices, 2nd ed., Academic Press, Boston, 1991.

[Ok1] A. Okounkov, Infinite wedge and measures on partitions, available via http://xxx.lanl.gov/abs/math/9907127. 
[Ok2] A. Okounkov, Random matrices and random permutations, available via http://xxx.lanl.gov/abs/math/9903176.

[Ol] G. Olshanski, Point processes and the infinite symmetric group. Part I: The general formalism and the density function, available via http://xxx.lanl.gov/abs/math/9804086.

[PS] L. Pastur and M. Shcherbina, Universality of the local eigenvalue statistics for a class of unitary invariant random matrix ensembles, J. Stat. Phys. 86, 109-147, 1997.

[PS1] M.Prähofer and H. Spohn, Statistical self-similarity of one-dimensional growth processes, available via http://xxx.lanl.gov/abs/cond-mat/9910273.

[PS2] M. Prähofer and H. Spohn, Universal distributions for growth processes in $1+1$ dimensions and random matrices, available via http://xxx.lanl.gov/abs/cond-mat/9912264.

[RS] M. Reed and B. Simon, Methods of Modern Mathematical Physics, vols. I-IV, Academic Press, New York, 1975-1980.

[Sa] B. Sagan, The Symmetric Group, Brooks/Cole Publ. Comp., 1991.

[S1] B. Simon, Trace Ideals and Their Applications, Cambridge University Press, New York, 1979.

[S2] B. Simon, The classical moment problem as a self-adjoint finite difference operator, Adv. in Math, 137, No. 1, 82-203, 1998..

[S3] B. Simon, personal communications.

[SCSS] H.-J. Sommers, A. Crisanti, H. Sompolinsky, and Y. Stein, Spectrum of large random asymmetric matrices, Phys. Rev. Lett. 60, 1895-1898, 1988.

[So1] A. Soshnikov, Level spacings distribution for large random matrices: Gaussian fluctuations, Ann. of Math. 148, 573-617, 1998. 
[So2] A. Soshnikov, Gaussian fluctuations in Airy, Bessel, sine and other determinantal random point fields, J. Stat. Phys., 100, No. 3/4, 491-522, 2000, available via http://xxx.lanl.gov/abs/math/9907012.

[So3] A. Soshnikov, Central limit theorem for local linear statistics in classical compact groups and related combinatorial identities, to appear in the Ann. of Probab., available via http://xxx.lanl.gov/abs.math/9908063.

[So4] A. Soshnikov, Universality at the edge of the spectrum in Wigner random matrices, Commun. Math. Phys., 207, No. 3, 697-733, 1999.

[Sp] H. Spohn, Interacting Brownian particles: A study of Dyson's model, in Hydrodynamic Behavior and Interacting Particle Systems, G. Papanicolau (ed.), Springer-Verlag, New York, 1987.

[St] R.P. Stanley, Enumerative Combinatorics, vol. 2, Cambridge University Press, 1999.

[TW1] C.A. Tracy and H. Widom, Correlation functions, cluster functions and spacing distribution for random matrices, J. Stat. Phys. 92, Nos. 5/6, 809-835, 1998.

[TW2] C.A. Tracy and H. Widom, Fredholm determinants, differential equations and matrix models, Commun. Math. Phys. 163, 3372, 1994.

[TW3] C.A. Tracy and H. Widom, Level-spacing distributions and the Airy kernel, Commun. Math. Phys. 159, 151-174, 1994.

[TW4] C.A. Tracy and H. Widom, Level spacing distributions and the Bessel kernel, Commun. Math. Phys. 161, 289-309, 1994.

[TW5] C.A. Tracy and H. Widom, Random unitary matrices, permutations and Painlevé, Commun. Math. Phys. 207, No. 3, 665-685, 1999.

[TW6] C.A. Tracy and H. Widom, On the distribution of the lengths of the longest monotone subsequences in random words, available via http://xxx.lanl.gov/abs/math/9904042. 
[We] H. Weyl, The Classical Groups: Their Invariants and Representations, Princeton Univ. Press, Princeton, 1939. 